\title{
DECAY OF CORRELATIONS FOR MAPS WITH UNIFORMLY CONTRACTING FIBERS AND LOGARITHM LAW FOR SINGULAR HYPERBOLIC ATTRACTORS.
}

\author{
VITOR ARAUJO, STEFANO GALATOLO, AND MARIA JOSE PACIFICO
}

\begin{abstract}
We consider maps preserving a foliation which is uniformly contracting and a onedimensional associated quotient map having exponential convergence to equilibrium (iterates of Lebesgue measure converge exponentially fast to physical measure). We prove that these maps have exponential decay of correlations over a large class of observables.

We use this result to deduce exponential decay of correlations for suitable Poincaré maps of a large class of singular hyperbolic flows. From this we deduce a logarithm law for these flows.
\end{abstract}

\section{Contents}

1. Introduction

1.1. Statement of the results

1.2. Organization of the text

2. Decay of correlations for fiber contracting maps

2.1. The Wasserstein-Kantorovich distance

2.2. Distance and disintegration

2.3. Exponential convergence to equilibrium and decay of correlations.

3. $p$-bounded variation and $C^{1+\alpha}$ piecewise expanding maps

4. Singular-hyperbolic attractors

4.1. Preliminary results

4.2. Cross-sections and Poincaré maps

4.3. Global Poincaré map

4.4. The two-dimensional map $F$

4.5. Integrability of $\tau, \log \left|T^{\prime}\right|$ and $\log \left|\partial_{y} G\right|$ with respect to the physical measures

5. Decay of correlation for the Poincaré maps.

6. Exact dimensionality

7. Logarithm law for singular hyperbolic attractors

7.1. Logarithm law for the flow

8. Notation of norms used throughout the paper

8.1. Custom norms used

8.2. Other norms we use or mention

References

Date: July 25, 2018.

2010 Mathematics Subject Classification. Primary: 37C10, 37C45, Secondary: 37C40, 37D30, 37D25.

Key words and phrases. singular flows, singular-hyperbolic attractor, exponential decay of correlations, exact dimensionality, logarithm law.

V.A. and M.J.P. were partially supported by CNPq, PRONEX-Dyn.Syst., FAPERJ, Balzan Research Project of J.Palis . 


\section{INTRODUCTION}

The term statistical properties of a dynamical system $F: X \rightarrow X$, where $X$ is a measurable space and $F$ a measurable map, refers to the behavior of large sets of trajectories of the system. It is well known that this relates to the properties of the evolution of measures by the dynamics.

Statistical properties are often a better object to be studied than pointwise behavior. In fact, the future behavior of initial data can be unpredictable, but statistical properties are often regular and their description simpler. Suitable results can be established in many cases, to relate the evolution of measures with that of large sets of points (ergodic theorems, large deviations, central limit, logarithm law, etc...).

In this paper we take the point of view of studying the evolution of measures and its speed of convergence to equilibrium to understand the statistical properties of a class of dynamical systems. We consider fiber-contracting maps which are a skew-product whose base transformation has exponential convergence to equilibrium (a measure of how fast iterates of the Lesbegue measure converge to the physical measure) and deduce that the whole system has exponential decay of correlations. By this it is possible to obtain other consequences regarding the statistical behavior of the dynamics, as the logarithm law, which will be introduced below. The regularity required by the techiniques we use to prove decay of correlations is quite low, so we can apply it to a class of systems including Poincaré return maps of Lorenz like systems.

We apply indeed these results to suitable Poincaré maps of an open dense subset of a large class of attractors of three-dimensional partially hyperbolic flows, including the singular-hyperbolic (or Lorenz-like) attractors, introduced in [35. These last kind of attractors present equilibria accumulated by regular orbits, and the Lorenz attractor as well the geometric Lorenz attractor are the most studied examples of this class of attractors [32, 22.

The main feature of these systems we exploit to estimate the decay of correlations is the existence of a quite regular stable foliation. We can disintegrate a measure along the foliation, and estimate the convergence of its iterates separately, along the stable and along the unstable direction, also by the use of suitable anysotropic norms.

Let us mention that it was proved in [34] that a generic $C^{1}$ vector field on a closed 3-manifold either has infinitely many sinks or sources, or else its non-wandering set admits a finite decomposition into compact invariant sets, each one being either a uniformly hyperbolic set or a singularhyperbolic attractor or a singular-hyperbolic repeller (which is a singular-hyperbolic attractor for the time reversed flow). This shows that the class of singular-hyperbolic attractors is a good representative of the limit dynamics of many flows on three-dimensional manifolds. More important, the class of singular-hyperbolic attractors contains every $C^{1}$ robustly transitive isolated set for flows on compact three-manifolds; see [35, 6].

We obtain exponential decay of correlations for the Poincaré return maps of a well-chosen finite family of cross-sections for these flows. In [18, exponential decay of correlations and limit laws for the two-dimensional Poincaré first return map of a class of geometrical Lorenz attractors were proved. We now provide similar results in a much more general context. We remark that our results on decay of correlations are finer that the one of Bunimovich [13] and Afraimovich, Chernov, Sataev [3], which can be seen as developments on the work of Pesin [38].

From the decay of correlations we then deduce a logarithm law for the hitting times to small targets in these cross-sections. Then, exploiting the fact that the flow on these attractors can be seen as a suspension flow over Poincaré return maps, we also obtain a logarithm law for the hitting time for the singular hyperbolic flow itself. We observe that quantitative dynamical properties of this class of systems are still quite unknown.

A logarithm law is a statement on the time a typical orbit hits a sequence of decreasing targets which is strictly related to the so called dynamical Borel Cantelli lemma and to diophantine approximation (see [19] and [16] for relations with the Borel Cantelli lemma and other approaches). Roughly speaking, a system has a logarithm law for a decreasing sequence of targets $S_{i}$ if the time which is needed for a typical orbit to hit the $i$-target is in some sense inversely proportional to the measure of the target. Hence it is a quantitative statement indicating how fast a typical orbit "fills" the space. Hitting time results of this kind have been proved in many continuous time 
dynamical systems of geometrical interest: geodesic flows, unipotent flows, homogeneous spaces etc.; see e.g. 9, 24, 30, 47, 17, 20. Other examples of connections with diophantine approximation can be found in [19] and 29]. We also remark that, in the symbolic setting, similar results about the hitting time are also used in information theory (see e.g. [44, 31]).

The logarithm law automatically holds for fastly mixing systems (see e.g, Theorem 11), but there are mixing systems for which the law does not hold ([19]). As it will be mentioned in the next section, some subclass of singular hyperbolic flows has been proved to be fastly mixing but in general the speed of mixing (and several statistical properties) of such flows are still unknown.

The referee of the current paper has pointed out to us that, in the case of Poincare maps for geometric Lorenz attractors, as considered in [18, exponential decay of correlations can be also obtained as an application of the landmark work of Young [48. Indeed, results on the statistical behavior of more or less general classes of Lorenz like flows have also been obtained by Markov tower like constructions; see e.g. [26. The approach we present here to estimate decay of correlations decouples the statistical properties of the base map of a skew-product with contracting fibers from the rest of the estimation about the speed of mixing. In the application to singular hyperbolic flows, we use the functional analytic approach to estimate the convergence to equilibrium for the base map (a piecewise expanding map), but in other cases also tower like constructions could be used in this step. We also remark that we need very weak regularity assumptions on the base map apart from the speed of convergence to equilibrium; see Section 2.3 and Remark 6. This will be essential when studying higher-dimensional partially hyperbolic systems, like the sectional-hyperbolic attractors introduced by Bonatti, Pumarino, Viana in [11] and Metzger-Morales in 33, where the quotient map over the contracting fibers in general fails even to have a derivative; it should be not more than a locally Holder homeomorphism.

1.1. Statement of the results. In Section 2 we establish results on the decay of correlations and convergence to equilibrium for fiber contracting maps with a base transformation which rapidly converges to equilibrium. Those results imply the following statement (which in turn will be applied to singular hyperbolic attractors).

We denote by $Q=\mathbb{I} \times \mathbb{I}$ the unit square, where $\mathbb{I}=[0,1]$. For a function $g: Q \rightarrow \mathbb{R}$ we denote by $L(g)$ the best Lipschitz constant of $g$, that is, $L(g)=\sup _{p, q \in Q} \frac{|g(p)-g(q)|}{|p-q|}$ where $|\cdot|$ is the Euclidean distance. We define the Lipschitz norm by setting $\|g\|_{l i p}=\|g\|_{\infty}+L(g)$ where, as usual, $\|g\|_{\infty}=\operatorname{ess}_{\sup _{p \in Q}}|g(p)|$ and $\operatorname{set} \operatorname{Lip}(Q)=\left\{g: Q \rightarrow \mathbb{R}:\|g\|_{l i p}<\infty\right\}$.

Theorem A. Let us consider a map $F: Q \circlearrowleft$ from the unit square into itself such that:

(1) $F$ has the form $F(x, y)=(T(x), G(x, y)$ ) (is a skew-product and preserves the natural vertical foliation of the square) ;

(2) $\left.F\right|_{\gamma}$ is $\lambda$-Lipschitz with $\lambda<1$ (hence is uniformly contracting) on each leaf $\gamma$ of the vertical foliation of the square;

(3) $\operatorname{var}^{\square}(G)<\infty$ (a kind of variation in one direction; see beginning of Section 3 for the definition),

(4) $T: \mathbb{I} \circlearrowleft$ is piecewise monotonic, with $n+1, C^{1}$ increasing branches on the intervals $\left(0, c_{1}\right), \ldots,\left(c_{i}, c_{i+1}\right), \ldots,\left(c_{n}, 1\right)$ and $\inf _{x \in \mathbb{I}}\left|T^{\prime}(x)\right|>1$.

(5) $\frac{1}{T^{\prime}}$ has finite universal $p$-bounded variation (a generalization of the notion of bounded variation; see again Section 3 for the definition);

(6) T has only one absolutely continuous (w.r.t. Lebesgue on $\mathbb{I}$ ) invariant probability measure (a.c.i.m.) for which it is weakly mixing.

Then the unique physical measure $\mu_{F}$ of $F$ has exponential decay of correlation with respect to Lipschitz observables, that is, there are $C, \Lambda \in \mathbb{R}^{+}, \Lambda<1$, such that

$$
\left|\int f \cdot\left(g \circ F^{n}\right) d \mu_{F}-\int g d \mu_{F} \int f d \mu_{F}\right| \leq C \Lambda^{n}\|g\|_{L i p}\|f\|_{L i p}, \quad f, g \in \operatorname{Lip}(Q) .
$$


The notion of physical measure is central in smooth Ergodic Theory. We say that a $F$-invariant probability measure $\mu_{F}$ is physical if the ergodic basin of $\mu_{F}$

$$
B\left(\mu_{F}\right)=\left\{p \in Q: \lim _{n \rightarrow+\infty} \frac{1}{n} \sum_{j=0}^{n-1} \varphi\left(F^{j}(p)\right)=\int \varphi d \mu_{F}, \text { for all continuous } \varphi: Q \rightarrow \mathbb{R}\right\}
$$

has positive area in $Q$ (two-dimensional Lebesgue measure).

Remark 1. We remark that items (4) to (6) of the assumptions on the above theorem can be replaced by (much more general) exponential convergence to equilibrium on the base map under suitable observables: see Theorem 1 and Theorem 3 in Section [. We also remark that Lipschitz norms can be replaced by suitable weaker anisotropic norms.

Next, we establish a logarithm law for the dynamics of the flow. This is a relation between hitting time to small targets and the local dimension of the invariant measure we consider. Let us consider a family of target sets $B_{r}\left(x_{0}\right)$, where $x_{0}$ is a given point, indexed by a real parameter $r$, and let us denote the time needed for the orbit of a point $x$ to enter in $B_{r}\left(x_{0}\right)$ by

$$
\tau_{F}\left(x, B_{r}\left(x_{0}\right)\right)=\min \left\{n \in \mathbb{N}^{+}: F^{n}(x) \in B_{r}\left(x_{0}\right)\right\} .
$$

A logarithm law is the statement that as $r \rightarrow 0$ the hitting time scales like $1 / \mu\left(B_{r}\right)$.

To express this, let us consider the local dimensions of a measure $\mu$

$$
\bar{d}_{\mu}\left(x_{0}\right)=\limsup _{r \rightarrow 0} \frac{\log \mu\left(B_{r}\left(x_{0}\right)\right)}{\log (r)} \text { and } \quad \underline{d}_{\mu}\left(x_{0}\right)=\liminf _{r \rightarrow 0} \frac{\log \mu\left(B_{r}\left(x_{0}\right)\right)}{\log (r)}
$$

representing the scaling rate of the measure of small balls as the radius goes to 0 . When the above limits coincide for $\mu$-almost every point, we say that $\mu$ is exact dimensional and set $d_{\mu}=\underline{d}_{\mu}(x)=$ $\bar{d}_{\mu}(x)$. From the main results of [15, 14, 46] it follows that in the kind of systems mentioned above (under mild extra conditions on the second coordinate $G$ of $F$ ) $\mu_{F}$ is exact dimensional, and the logarithm law holds.

Proposition 1. If $F(x, y)=(T(x), G(x, y))$ is as in the setting of Theorem $\AA$ and

- $F$ is injective;

- $G$ is $C^{1}$ on $J \times \mathbb{I}$, where $J$ is any monotonicity interval of $T$;

- $|\partial G / \partial y|>0$ whenever defined, $\sup |\partial G / \partial x|<\infty$ and $\sup |\partial G / \partial y|<1$;

then the physical measure $\mu_{F}$ is exact dimensional and for $\mu_{F}$-almost every $x$ it holds

$$
\lim _{r \rightarrow 0} \frac{\log \tau_{F}\left(x, B_{r}\left(x_{0}\right)\right)}{-\log r}=d_{\mu_{F}}\left(x_{0}\right) .
$$

We now apply these results to three-dimensional flows having a singular-hyperbolic attractor. Let $S H^{2}\left(M^{3}\right)$ be the family of all $C^{2}$ vector fields $X$ on a compact three-manifold $M^{3}$ having an open trapping region $U$, i.e., $\overline{X_{t}(U)} \subset U$ for all $t>0$, such that its maximal invariant subset $\Lambda=\cap_{t>0} X_{t}(U)$ is a compact transitive singular-hyperbolic set.

This means that, for each $X \in S H^{2}\left(M^{3}\right)$, there exists a continuous invariant and dominated decomposition $E_{\Lambda}^{s} \oplus E_{\Lambda}^{c}$ of the tangent space at each $x \in \Lambda$, where $E_{\Lambda}^{s}$ is a one-dimensional subbundle uniformly contracted by the derivative $D X_{t}$, and $E_{\Lambda}^{c}$ is a two-dimensional sub-bundle, containing the flow direction, and whose area is uniformly expanded by the action of $D X_{t}$, that is, there are constants $C, \lambda>0$ such that $\left|\operatorname{det} D X_{t}\right| E_{x}^{c} \mid \geq C e^{\lambda t}$. In addition, there are finitely many hyperbolic singularities $\sigma$ in $\Lambda$, which are Lorenz-like, i.e., the eigenvalues $\lambda_{i}(\sigma), 1 \leq i \leq 3$, of $D X(\sigma)$ are real and satisfy $\lambda_{2}(\sigma)<\lambda_{3}(\sigma)<0<-\lambda_{3}(\sigma)<\lambda_{1}(\sigma)$ (see Section 4 for a more precise description). We consider the $C^{2}$ topology of vector fields in $S H^{2}\left(M^{3}\right)$ in what follows.

Given a flow having a singular hyperbolic attractor, there exists a finite family $\Xi$ of welladapted cross-sections of the flow where we can define a Poincaré return map $F$ which satisfies the properties in the statement of Theorem $\mathrm{A}$ after a suitable choice of coordinates. To take advantage of the above cited result from Steinberger [46] on exact dimensionality and obtain Proposition 1 , we need that the Poincaré return map $F$ be injective, which is not evident in the construction 
and choice of these cross-sections done at [7. Because of this, the construction presented here is slightly different from the ones presented elsewhere.

Once constructed a suitable section, taking some nonresonance conditions on the eigenvalues of the equilibria of $X$ inside $\Lambda$ we can reduce the dynamics on $\Lambda$ to a map $F$ as in Theorem $\mathrm{A}$ and obtain

Corollary 1. There exists an open dense set $\mathcal{A}$ of vector fields (satisfying a nonresonance condition) in $S H^{2}\left(M^{3}\right)$ such that, for each $X \in \mathcal{A}$, we can find a finite family $\Xi$ of cross-sections to the flow $X_{t}$ of $X$ such that an iterate of the Poincare first return map $F: \operatorname{dom}(F) \subset \Xi \rightarrow \Xi$ has a finite set of SRB measures $\mu_{F}^{i}$, each of them has exponential decay of correlations with respect to Lipschitz observables: there are $C, \Lambda \in \mathbb{R}^{+}, \Lambda<1$ satisfying for every pair $f, g: \Xi \rightarrow \mathbb{R}$ of Lipschitz functions

$$
\left|\int f \cdot\left(g \circ F^{n}\right) d \mu_{F}^{i}-\int g d \mu_{F}^{i} \int f d \mu_{F}^{i}\right| \leq C \Lambda^{n}|| g\left\|_{L i p}\right\| f \|_{L i p}, \quad n \geq 1 .
$$

We remark that, since the work of Ruelle [43], it is well-known that exponentially mixing for the base transformation of a suspension flow does not imply fast mixing for the suspension flow. In fact, the suspension flow might be mixing but without exponential decay of correlations as in [43]; or it may have arbitrarily slow decay of correlations, as shown by Pollicott in [39. Hence we cannot deduce in general any kind of fast mixing results for the flow on a singular-hyperbolic attractor from Corollary 1. However, in a recent work of one of the authors with Varandas 8, it has been proved the existence of a $C^{2}$ open subset of vector fields having a geometrical Lorenz attractor with exponential decay of correlations for the flow on $C^{1}$ observables, from which follows the exponential decay of correlations for the corresponding Poincaré map. But this $C^{2}$ open subset was obtained under very strong conditions which cannot hold in such generality as in Corollary 1 .

Under mild (open and dense) conditions on the eigenvalues of the hyperbolic singularities of $\Lambda$, we can show that the second coordinate $G$ of $F$ is in the setting of Proposition 1 Hence we can also deduce the logarithm law for the Poincaré return map; see Section 7.

We can then use this to obtain a log-law for the hitting times for the singular-hyperbolic flow, in a way similar to what was done in [18. Let $x, x_{0} \in \Lambda$ and

$$
\tau_{r}^{X_{t}}\left(x, x_{0}\right)=\inf \left\{t \geq 0 \mid X_{t}(x) \in B_{r}\left(x_{0}\right)\right\}
$$

be the time needed for the $X$-orbit of a point $x$ to enter for the first time in a ball $B_{r}\left(x_{0}\right)$. The number $\tau_{r}^{X_{t}}\left(x, x_{0}\right)$ is the hitting time associated to the flow $X_{t}$ and target $B_{r}\left(x_{0}\right)$. Let us consider $d_{\mu_{X}}\left(x_{0}\right)$, the local dimension at $x_{0}$ of the unique physical measure $\mu_{X}$ supported on the singular-hyperbolic attractor (which was constructed in [7).

Corollary 2. If $X_{t}$ is a flow over a singular-hyperbolic attractor in the setting of Corollary 1 and if, in addition, the eigenvalues of every equilibrium point $\sigma$ of $X$ in $\Lambda$ satisfy $\lambda_{1}(\sigma)+\lambda_{2}(\sigma)<0$ (which includes the classical Lorenz system of ODEs), then for each regular point $x_{0} \in \Lambda$ such that $d_{\mu_{X}}\left(x_{0}\right)$ exists, we have

$$
\lim _{r \rightarrow 0} \frac{\log \tau_{r}^{X_{t}}\left(x, x_{0}\right)}{-\log r}=d_{\mu_{X}}\left(x_{0}\right)-1
$$

for $\mu_{X}$-almost each $x \in \Lambda$.

1.2. Organization of the text. We begin by proving exponential decay of correlations for fibercontracting maps in Section 2. We use the exponential convergence to equilibrium of the base transformation to prove exponential convergence to equilibrium of the fibered contracting map. For this we compare the disintegrations of the push-forward measures along the fibers using the Wasserstein-Kantorovich distance between measures. We choose appropriate norms to be able to compare the size of the correlation functions and deduce the exponential decay of correlations from the exponential convergence to equilibrium. To help the reader follow the diverse norms used throughout we have compiled a list of norms in the last Section 8 .

In Section 3, we explain the meaning of generalized $p$-bounded variation and the existence of absolutely continuous invariant probability measures for $C^{1+\alpha}$ piecewise expanding maps (not 
necessarily Markov maps) whose densities are of generalized $p$-bounded variation. This is based on the work of Keller in 28.

We present singular-hyperbolic attractors and the related properties which we use in the remaining part of the paper in Section 4, mainly following the works 7 and 6].

In Section 5. we analyze the Poincaré return map associated to a singular-hyperbolic attractor and show that this map satisfies the conditions needed to deduce exponential decay of correlations for Lipschitz observables. Here we take advantage of the constructions presented in the previous section.

In Section 6 we also show that the physical measure of the flow is exact dimensional. This is done using the results of [46].

Following [14, 15, 18, in Section 7 we use the results proved in the previous sections to establish the logarithm law for the hitting times for the Poincaré map and flow of a singular-hyperbolic attractor with mild conditions on the eigenvalues of its hyperbolic singularities.

Acknowledgments: S. G. wishes to thank IMPA, PUC, and UFRJ (Rio de Janeiro), where a part of this work has been done, for their warm hospitality. All authors whish to thank Carlangelo Liverani for illuminating discussions about convergence to equilibrium and decay of correlations.

\section{DECAY OF CORRELATIONS FOR FIBER CONTRACTING MAPS}

In this section we will prove that a certain class of maps, with contracting fibers and low regularity have exponential decay of correlation. This class contains suitable Poincaré maps of singular-hyperbolic attractors as we will se in what follows.

The methods we use are related both to coupling and to spectral techniques, and will be implemented by adequate anisotropic norms.

We will quantify the speed of convergence of the iterates of the Lesbegue measure to the physical invariant measure through the help of a certain distance on the space of probability measures.

2.1. The Wasserstein-Kantorovich distance. If $Y$ is a bounded metric space, we denote by $P M(Y)$ the set of Borel probability measures on $Y$. We denote by $L(g)$ the best Lipschitz constant of $g: Y \rightarrow \mathbb{R}$, that is, $L(g)=\sup _{x, y} \frac{|g(x)-g(y)|}{|x-y|}$ and set $\|g\|_{l i p}=\|g\|_{\infty}+L(g)$.

Let us consider the following notion of distance between measures: given two probability measures $\mu_{1}$ and $\mu_{2}$ on $Y$

$$
W_{1}\left(\mu_{1}, \mu_{2}\right)=\sup \left\{\left|\int_{Y} g d \mu_{1}-\int_{Y} g d \mu_{2}\right|: g: Y \rightarrow \mathbb{R}, L(g)=1\right\} .
$$

We remark that adding a constant to the test function $g$ does not change the above difference of integrals.

We also recall that if $F: X \rightarrow Y$ is a map, then it induces an associated map $F^{*}: P M(X) \rightarrow$ $P M(Y)$ defined as $\left(F^{*}(\mu)\right)(A)=\mu\left(F^{-1}(A)\right)$.

The above defined distance has the following basic properties; see e.g [4, Prop 7.1.5] and [18.

Proposition 2. $W_{1}$ is a distance and if $Y$ is separable and complete, then $P M(Y)$ with this distance becomes a separable and complete metric space. A sequence is convergent for the $W_{1}$ metrics if and only if it is convergent for the weak-star topology. Moreover, let $F: \gamma \rightarrow \gamma$ be a $\lambda$-contracting map, let us consider two probability measures $\mu, \nu$ on $\gamma$. Then

$$
W_{1}\left(F^{*}(\mu), F^{*}(\nu)\right) \leq \lambda \cdot W_{1}(\mu, \nu) .
$$

Remark 2. (distance and convex combinations) If $\sum_{1}^{n} a_{i}=1, a_{i} \geq 0, \mu_{i}, \nu_{i} \in P M(Y)$ for $i=1, \ldots, n$, then

$$
W_{1}\left(\sum_{1}^{n} a_{i} \mu_{i}, \sum_{1}^{n} a_{i} \nu_{i}\right) \leq \sum_{1}^{n} a_{i} \cdot W_{1}\left(\mu_{i}, \nu_{i}\right) .
$$


Remark 3. If $g$ is $\ell$-Lipschitz and $\mu_{1}, \mu_{2}$ are probability measures then

$$
\left|\int_{Y} g d \mu_{1}-\int_{Y} g d \mu_{2}\right| \leq \ell \cdot W_{1}\left(\mu_{1}, \mu_{2}\right) .
$$

Remark 4. We will see that the decay of correlation of the system is related to the speed of convergence of iterates of a starting measure to the invariant one. In 18 the rate of convergence with respect to the $W_{1}$ distance was considered to obtain exponential decay of correlation for the Poincaré map of a geometric Lorenz flow. Here, being in a more general case, having less regularity, we cannot perform the same construction. But this idea will be part of the construction we are going to implement.

We will also use the following variation distance on the space of probability measures

$$
V\left(\mu_{1}, \mu_{2}\right)=\sup \left\{\left|\int h d \mu_{1}-\int h d \mu_{2}\right|:\|h\|_{\infty} \leq 1\right\} .
$$

We remark that when the two measures have a density, this is the $L^{1}$ distance between the densities.

2.2. Distance and disintegration. We denote by $\mathbb{I}=[0,1]$ and $Q=\mathbb{I} \times \mathbb{I}$. We consider the sup distance on $Q$ so that the diameter is one: $\operatorname{diam}(Q)=1$. This choice is not essential, but will avoid the presence of many multiplicative constants in the following, making notations cleaner.

The square $Q$ will be foliated by stable, vertical leaves. We will denote the leaf with $x$ coordinate by $\gamma_{x}$ or, with a small abuse of notation, when no confusion is possible, we will denote both the leaf and its coordinate by $\gamma$.

Let $f \mu$ be the measure $\mu_{1}$ such that $d \mu_{1}=f d \mu$. Let $\mu$ be a probability measure on $Q$. Such measures on $Q$ will be often disintegrated in the following way: for each Borel set $A$

$$
\mu(A)=\int_{\gamma \in I} \mu_{\gamma}(A \cap \gamma) d \mu_{x}
$$

with $\mu_{\gamma}$ being probability measures on the leaves $\gamma$ and $\mu_{x}$ the marginal on the $x$ axis, which will be absolutely continuous with respect to the Lebesgue length measure. We denote by $\phi_{x}$ the density of $\mu_{x}$.

Now we consider the integral of a suitable observable $g: Q \rightarrow \mathbb{R}$. Let us consider the following anisotropic norm, considering Lipschitz regularity only on the vertical direction. Let $\|\cdot\|_{\uparrow l i p}$ be defined by

$$
\|g\|_{\uparrow l i p}=\|g\|_{\infty !}+\operatorname{Lip}_{y}(g)
$$

where for simplicity we consider

$$
\|g\|_{\infty}:=\sup _{x, y \in[0,1]}|g(x, y)| \text { and } \operatorname{Lip}_{y}(g):=\sup _{\substack{x, y_{1}, y_{2} \in[0,1] \\ y_{1} \neq y_{2}}} \frac{\left|g\left(x, y_{2}\right)-g\left(x, y_{1}\right)\right|}{\left|y_{2}-y_{1}\right|} .
$$

If $\mu^{1}$ and $\mu^{2}$ are two disintegrated measures as above, the integral of an observable $g$ can be estimated as function of the above norm, some distance between their respective marginals on the $x$ axis and measures on the leaves.

Proposition 3. Let $\mu^{1}, \mu^{2}$ be measures on $Q$ as above, such that for each Borel set $A$

$$
\mu^{1}(A)=\int_{\gamma \in I} \mu_{\gamma}^{1}(A \cap \gamma) d \mu_{x}^{1} \quad \text { and } \quad \mu^{2}(A)=\int_{\gamma \in I} \mu_{\gamma}^{2}(A \cap \gamma) d \mu_{x}^{2},
$$

where $\mu_{x}^{i}$ is absolutely continuous with respect to the Lebesgue measure. In addition, let us assume that

(1) $\int_{I} W_{1}\left(\mu_{\gamma}^{1}, \mu_{\gamma}^{2}\right) d \mu_{x}^{1} \leq \varepsilon$

(2) $V\left(\mu_{x}^{1}, \mu_{x}^{2}\right) \leq \delta$. 
Ther $\left|\int g d \mu^{1}-\int g d \mu^{2}\right| \leq\|g\|_{\uparrow l i p}(\varepsilon+\delta)$.

Proof. Disintegrating $\mu^{1}$ and $\mu^{2}$ we get

$$
\left|\int g d \mu^{1}-\int g d \mu^{2}\right|=\left|\int_{\gamma \in \mathbb{I}} \int_{\gamma} g d \mu_{\gamma}^{1} d \mu_{x}^{1}-\int_{\gamma \in \mathbb{I}} \int_{\gamma} g d \mu_{\gamma}^{2} d \mu_{x}^{2}\right| .
$$

Adding and subtracting $\iint_{\gamma} g d \mu_{\gamma}^{2} d \mu_{x}^{1}$ the last expression is equivalent to

$$
\left|\int_{\mathbb{I}} \int_{\gamma} g d \mu_{\gamma}^{1} d \mu_{x}^{1}-\int_{\mathbb{I}} \int_{\gamma} g d \mu_{\gamma}^{2} d \mu_{x}^{1}+\int_{\mathbb{I}} \int_{\gamma} g d \mu_{\gamma}^{2} d \mu_{x}^{1}-\int_{\mathbb{I}} \int_{\gamma} g d \mu_{\gamma}^{2} d \mu_{x}^{2}\right| .
$$

This is bounded by (see Remark 3)

$$
\begin{gathered}
\left|\int_{\mathbb{I}}\left(\int_{\gamma} g d \mu_{\gamma}^{1}-g d \mu_{\gamma}^{2}\right) d \mu_{x}^{1}\right|+\left|\int_{\mathbb{I}} \int_{\gamma} g d \mu_{\gamma}^{2} d \mu_{x}^{1}-\int_{\mathbb{I}} \int_{\gamma} g d \mu_{\gamma}^{2} d \mu_{x}^{2}\right| \\
\leq\|g\|_{\uparrow l i p} \varepsilon+\left|\int_{\mathbb{I}} \int_{\gamma} g d \mu_{\gamma}^{2} d \mu_{x}^{1}-\int_{\mathbb{I}} \int_{\gamma} g d \mu_{\gamma}^{2} d \mu_{x}^{2}\right| \\
\leq \varepsilon\|g\|_{\uparrow l i p}+\left|\int_{\mathbb{I}} \int_{\gamma} g d \mu_{\gamma}^{2} d\left(\mu_{x}^{1}-\mu_{x}^{2}\right)\right| .
\end{gathered}
$$

For almost each $\gamma$ it holds $h(\gamma)=\left|\int_{\gamma} g d \mu_{\gamma}^{2}\right| \leq\|g\|_{\uparrow l i p}$. Thus, by assumption (2) in the statement, the proposition is proved.

2.3. Exponential convergence to equilibrium and decay of correlations. Let us consider a manifold $M$ (possibly with boundary) and the dynamics on $M$ generated by the iteration of a function $T: M \rightarrow M$. We will consider a notion of speed of approach of an absolutely continuous initial measure, with density $f$, to an invariant measure $\mu$. Of course, many generalizations are possible, but we will consider here only absolutely continuous measures as starting measures.

Definition 1. We say that $T$ has exponential convergence to equilibrium with respect to norms $\|\cdot\|_{a}$ and $\|\cdot\|_{b}$, if there are $C, \Lambda \in \mathbb{R}^{+}, \Lambda<1$ such that for $f \in L^{1}(m), g \in L^{1}(\mu)$

$$
\left|\int f \cdot\left(g \circ T^{n}\right) d m-\int g d \mu \int f d m\right| \leq C \Lambda^{n} \cdot\|g\|_{a} \cdot\|f\|_{b}, \quad n \geq 1
$$

where $m$ is the Lebesgue measure on $M$.

We remark that in several situations, exponential convergence to equilibrium can be obtained as consequence of spectral gap of the transfer operator restricted to suitable funcion spaces, see Lemma 5

Now we consider maps preserving a regular foliation, which contracts the leaves and whose quotient map (the induced map on the space of leaves) has exponential convergence to equilibrium. We will give an estimation of the speed of convergence to equilibrium for this kind of maps, establishing that it is also exponential.

Definition 2. If $f: Q \rightarrow \mathbb{R}$ is integrable, we denote by $\pi(f): \mathbb{I} \rightarrow \mathbb{R}$ the function $\pi(f): x \mapsto$ $\int_{\mathbb{I}} f(x, t) d t$.

Theorem 1. Let $F: Q \circlearrowleft$ be a Borel function such that $F(x, y)=(T(x), G(x, y))$. Let $\mu$ be an $F$-invariant measure with marginal $\mu_{x}$ on the $x$-axis which, moreover, is T-invariant. Let us suppose that

(1) $\left(T, \mu_{x}\right)$ has exponential convergence to equilibrium with respect to the norm $\|\cdot\|_{\infty}$ (the $L^{\infty}$ norm) and to a norm which we denote by $\|\cdot\|_{-}$.

(2) $T$ is nonsingular with respect to the Lesbegue measure, piecewise continuous and monotonic: there is a collection of intervals $\left\{I_{i}\right\}_{i=1, \ldots, m}, \cup I_{i}=I$ such that on each $I_{i}, T$ is an homeomorphism onto its image.

\footnotetext{
${ }^{1}$ We remark that to have the left hand of item (1) well defined we can assume (without changing $\mu^{1}$ ) that $\mu_{\gamma}^{2}$ is defined in some way, for example $\mu_{\gamma}^{2}=m$ (the one dimensional Lebesgue measure on the leaf) for each leaf where the density of $\mu_{x}^{2}$ is null.
} 
(3) $F$ is a contraction on each vertical leaf: $G$ is $\lambda$-Lipschitz in $y$ with $\lambda<1$.

Then $(F, \mu)$ has exponential convergence to equilibrium in the following sense. There are $C, \Lambda \in$ $\mathbb{R}^{+}, \Lambda<1$ such that

$$
\left|\int f \cdot\left(g \circ F^{n}\right) d m-\int g d \mu \int f d m\right| \leq C \Lambda^{n} \cdot\|g\|_{\uparrow l i p} \cdot\left(\|\pi(f)\|_{-}+\|f\|_{1}\right)
$$

for each $f \geq 0$.

Proof. Let us take an integrable non-negative $f: Q \rightarrow \mathbb{R}$ and an essentially bounded $g: Q \rightarrow \mathbb{R}$. We can divide and multiply by $\|g\|_{\uparrow l i p} \int f d m$ obtaining

$$
\left|\int g \circ F^{n} \cdot f d m-\int g d \mu \int f d m\right|=\left(\|g\|_{\uparrow l i p} \int f d m\right)\left|\int \frac{g \circ F^{n}}{\|g\|_{\uparrow l i p}} \cdot \frac{f}{\int f d m} d m-\int \frac{g}{\|g\|_{\uparrow l i p}} d \mu\right| .
$$

We denote $\nu^{n}=F^{* n}\left(\frac{f}{\int f d m} m\right)$ and disintegrate the measures. Let us suppose having iterated the system $n_{0}$ times, and then continue to iterate again $n$ more times. By item (1) after the first $n_{0}$ iterations, the marginals become exponentially close:

$$
\sup _{\|h\|_{\infty} \leq 1}\left|\int h d\left(\left(\nu^{n_{0}}\right)_{x}\right)-\int h d\left(\mu_{x}\right)\right| \leq C \Lambda^{n_{0}} \cdot\left\|\pi\left(\frac{f}{\int f d m}\right)\right\|_{-} .
$$

Now iterating $n$ more times we estimate the distance on the leaves, and then the speed of convergence by Theorem [3. Let us consider $\left\{\bar{I}_{i}\right\}_{i=1, \ldots, m^{\prime}}$, the intervals where the continuous branches of $T^{n}$ are defined. Let us consider $\varphi_{i}=1_{\bar{I}_{i} \times I}$ and $\nu_{i}=\varphi_{i} \nu^{n_{0}}, \mu_{i}=\varphi_{i} \mu$ so that $\mu=\sum \mu_{i}, \nu^{n_{0}}=\sum \nu_{i}$.

Remark that by item (1) after the first $n_{0}$ iterations, also the marginals of $\nu_{i}$ and $\mu_{i}$ are exponentially close, and since $\varphi_{i}$ have disjoint support:

$$
\sum_{i \leq m^{\prime}} \sup _{\|h\|_{\infty} \leq 1}\left|\int h d\left(\left(\nu_{i}\right)_{x}\right)-\int h d\left(\left(\mu_{i}\right)_{x}\right)\right| \leq C \Lambda^{n_{0}} \cdot\left\|\pi\left(\frac{f}{\int f d m}\right)\right\|_{-} .
$$

By triangle inequality

$\left|\int \frac{g}{\|g\|_{\uparrow l i p}} d\left(F^{*\left(n+n_{0}\right)}(\nu)\right)-\int \frac{g}{\|g\|_{\uparrow l i p}} d(\mu)\right| \leq \sum_{i=1, \ldots, m}\left|\int \frac{g}{\|g\|_{\uparrow l i p}} d\left(F^{* n}\left(\nu_{i}\right)\right)-\int \frac{g}{\|g\|_{\uparrow l i p}} d\left(F^{* n}\left(\mu_{i}\right)\right)\right|$.

Let us denote $T_{i}=\left.T^{n}\right|_{\bar{I}_{i}}$, remark that this is injective. Then by Proposition 3

$$
\begin{aligned}
& \left|\int \frac{g}{\|g\|_{\uparrow l i p}} d\left(F^{* n}\left(\nu_{i}\right)\right)-\int \frac{g}{\|g\|_{\uparrow l i p}} d\left(F^{* n}\left(\mu_{i}\right)\right)\right| \\
& \leq \int_{I} W_{1}\left(\left(F^{* n} \mu_{i}\right)_{\gamma},\left(F^{* n} \nu_{i}\right)_{\gamma}\right) d T^{* n}\left(\left(\nu_{i}\right)_{x}\right)+\left\|T^{* n}\left(\left(\mu_{i}\right)_{x}\right)-T^{* n}\left(\left(\nu_{i}\right)_{x}\right)\right\|_{1} \\
& \leq \int_{I} W_{1}\left(F^{* n}\left(\left(\mu_{i}\right)_{T_{i}^{-1}(\gamma)}\right), F^{* n}\left(\left(\nu_{i}\right)_{T_{i}^{-1}(\gamma)}\right)\right) d T^{* n}\left(\left(\nu_{i}\right)_{x}\right)+\left\|T^{* n}\left(\left(\mu_{i}\right)_{x}\right)-T^{* n}\left(\left(\nu_{i}\right)_{x}\right)\right\|_{1}
\end{aligned}
$$

and by uniform contraction along the leaves we can bound

$$
\begin{aligned}
& \leq \lambda^{n} \int_{I} W_{1}\left(\left(\mu_{i}\right)_{T_{i}^{-1}(\gamma)},\left(\nu_{i}\right)_{T_{i}^{-1(\gamma)}}\right) d T^{* n}\left(\left(\nu_{i}\right)_{x}\right)+\left\|T^{* n}\left(\left(\mu_{i}\right)_{x}\right)-T^{* n}\left(\left(\nu_{i}\right)_{x}\right)\right\|_{1} \\
& =\lambda^{n} \int_{I_{i}} W_{1}\left(\left(\mu_{i}\right)_{\gamma},\left(\nu_{i}\right)_{\gamma}\right) d\left(\left(\nu_{i}\right)_{x}\right)+\left\|T^{* n}\left(\left(\mu_{i}\right)_{x}\right)-T^{* n}\left(\left(\nu_{i}\right)_{x}\right)\right\|_{1} .
\end{aligned}
$$

Summarizing, we obtain

$$
\begin{aligned}
& \left|\int \frac{g}{\|g\|_{\uparrow l i p}} d\left(F^{* n}\left(\nu_{i}\right)\right)-\int \frac{g}{\|g\|_{\uparrow l i p}} d\left(F^{* n}\left(\mu_{i}\right)\right)\right| \\
& \leq \lambda^{n} \sum_{i} \int_{I_{i}} W_{1}\left(\left(\mu_{i}\right)_{\gamma},\left(\nu_{i}\right)_{\gamma}\right) d\left(\left(\nu_{i}\right)_{x}\right)+\sum_{i}\left\|T^{* n}\left(\left(\mu_{i}\right)_{x}\right)-T^{* n}\left(\left(\nu_{i}\right)_{x}\right)\right\|_{1} \\
& =\lambda^{n} \int_{I} W_{1}\left(\mu_{\gamma}, \nu_{\gamma}^{n_{0}}\right) d\left(\nu_{x}\right)+\sum_{i}\left\|T^{* n}\left(\left(\mu_{i}\right)_{x}\right)-T^{* n}\left(\left(\nu_{i}\right)_{x}\right)\right\|_{1} .
\end{aligned}
$$


Since $T^{* n}$ is a $L^{1}$ contraction, from (2.6) this is less or equal than

$$
\begin{aligned}
\lambda^{n} \int_{I} W_{1}\left(\mu_{\gamma}, \nu_{\gamma}^{n_{0}}\right) d\left(\nu_{x}\right) & +\sum_{i}\left\|\left(\mu_{i}\right)_{x}-\left(\nu_{i}\right)_{x}\right\|_{1} \\
& \leq \lambda^{n} \int_{I} W_{1}\left(\mu_{\gamma}, \nu_{\gamma}^{n_{0}}\right) d\left(\nu_{x}\right)+C \Lambda^{n_{0}} \cdot\left\|\pi\left(\frac{f}{\int f d m}\right)\right\|_{-} .
\end{aligned}
$$

Finally we can deduce

$$
\begin{aligned}
& \left(\|g\|_{\uparrow \text { lip }} \int f d m\right)\left|\int \frac{g}{\|g\|_{\uparrow l i p}} d\left(F^{*\left(n+n_{0}\right)}\left(\frac{f}{\int f d m} m\right)\right)-\int \frac{g}{\|g\|_{\uparrow l i p}} d \mu\right| \\
& \leq\left(\|g\|_{\uparrow l i p} \int f d m\right)\left[\lambda^{n}+C \Lambda^{n_{0}} \cdot\left\|\pi\left(\frac{f}{\int f d m}\right)\right\|_{-}\right]
\end{aligned}
$$

which implies the statement and concludes the proof of Theorem 1 .

Remark 5. Since $\|f\|_{\infty} \geq\|f\|_{1}$ we can also state: under the assumptions of Theorem 1 , if $f \geq 0$

$$
\left|\int f \cdot\left(g \circ F^{n}\right) d m-\int g d \mu \int f d m\right| \leq C \Lambda^{n} \cdot\|g\|_{\uparrow l i p} \cdot\left(\|\pi(f)\|_{-}+\|f\|_{\infty}\right) .
$$

Remark 6. We remark that if, instead of exponential convergence to equilibrium for the base map $\left.\left(C \Lambda^{n_{0}} \cdot\left\|\pi\left(\frac{f}{\int f d m}\right)\right\|_{-}\right]\right)$, we had a slower rate of convergence $\left(C_{n_{0}} \cdot\left\|\pi\left(\frac{f}{\int f d m}\right)\right\|_{-}\right.$with $C_{n} \rightarrow 0$ slower than exponential), then because $\lambda^{n}$ will converge to 0 much faster than $C_{n}$ (see the last equation in the above proof), the same arguments lead to an estimation for the convergence to equilibrium for the skew product with the same kind of asymptotical behavior as $C_{n}$.

Now let us relate convergence to equilibrium to decay of correlations. We consider the general case of a measure preserving map.

Lemma 1 (convergence to equilibrium and decay of correlations). Let us consider a measurable map $F: \Omega \rightarrow \Omega$, two probability measures $m, \mu$ on $\Omega$, such that $\mu$ is invariant. If we have a convergence to equilibrium with speed $C_{n}$ and norms $\|\cdot\|_{1}$ and $\|\cdot\|_{2}$, that is

$$
\left|\int g\left(F^{n}(x)\right) f(x) d m-\int f(x) d m \int g(x) d \mu\right| \leq C_{n} \cdot\|f\|_{1} \cdot\|g\|_{2},
$$

then, for each $k,\left|\int f \cdot\left(g \circ F^{n}\right) d \mu-\int g d \mu \int f d \mu\right|$ is bounded by

$$
C_{k}\|1\|_{1}\left\|g \circ F^{n} f\right\|_{2}+C_{n}\left\|f \circ F^{k}\right\|_{1}\left\|g \circ F^{k}\right\|_{2}+C_{k}\left|\int g d \mu\right|\|f\|_{2}\|1\|_{1},
$$

where 1 is the constant function with value 1 .

Proof. Adding and subtracting we rewrite $\left|\int f \cdot\left(g \circ F^{n}\right) d \mu-\int g d \mu \int f d \mu\right|$ as

$$
\begin{aligned}
& \mid \int f \cdot\left(g \circ F^{n}\right) d \mu-\int 1 \cdot\left(g \circ F^{n+k}\right)\left(f \circ F^{k}\right) d m \\
& \quad+\int\left(g \circ F^{n+k}\right)\left(f \circ F^{k}\right) d m-\int f \circ F^{k} d m \int g d \mu \\
& \quad+\int\left(f \circ F^{k}\right) \cdot 1 d m \int g d \mu-\int g d \mu \int f d \mu \mid .
\end{aligned}
$$

Applying the assumption to each line we obtain the three summands in the statement.

Now we use the above results to deduce exponential decay of correlations from exponential speed of convergence to equilibrium.

Theorem 2. Let $F$ be a map satisfying the assumptions of Theorem 1 and let us suppose that there are $C_{1}, K \in \mathbb{R}$ and a seminorm $\|\cdot\|_{\square}$ such that for each $n \geq 1$

$$
\left\|\pi\left(f \circ F^{n}\right)\right\|_{-}+\left\|f \circ F^{n}\right\|_{\square} \leq C_{1} K^{n}\left(\|\pi(f)\|_{-}+\|f\|_{\uparrow l i p}+\|f\|_{\square}\right) .
$$


Then $F$ has exponential decay of correlations: there are $C_{2}, \Lambda \in \mathbb{R}^{+}, \Lambda<1$ such that for $n \geq 1$

$$
\left|\int f \cdot\left(g \circ F^{n}\right) d \mu-\int g d \mu \int f d \mu\right| \leq C_{2} \Lambda^{n}\|g\|_{\uparrow l i p}\left(\|f\|_{\uparrow l i p}+\|\pi(f)\|_{-}+\|f\|_{\square}\right) .
$$

Proof. Let us consider bounded observables $f, g$. Since adding a constant to $f$ the correlation integral does not change, we can suppose $f \geq 0$ and we can apply Theorem 1 .

By Theorem 1, Remark [5 and Lemma 1, there are $C, \Lambda \in \mathbb{R}^{+}, \Lambda<1$, s.t. for each $k$

$$
\begin{gathered}
\left|\int f \cdot\left(g \circ F^{n}\right) d \mu-\int g d \mu \int f d \mu\right| \leq C \Lambda^{k}\left(\|\pi(1)\|_{-}+\|1\|_{\infty}\right) \cdot\left\|f \cdot\left(g \circ F^{n}\right)\right\|_{\uparrow l i p}+ \\
C \Lambda^{n}\left(\left\|\pi\left(f \circ F^{k}\right)\right\|_{-}+\left\|f \circ F^{k}\right\|_{\infty}\right) \cdot\left\|g \circ F^{k}\right\|_{\uparrow l i p}+C \Lambda^{k}\left|\int g d \mu\right|\|f\|_{\uparrow l i p}\left(\|\pi(1)\|_{-}+\|1\|_{\infty}\right) .
\end{gathered}
$$

By assumption (2.8), we take $k=\lfloor\alpha n\rfloor$ (the integer part) with $\alpha$ so small that

$$
C \Lambda^{n}\left(\left\|\pi\left(f \circ F^{\lfloor\alpha n\rfloor}\right)\right\|_{-}+\left\|f \circ F^{\lfloor\alpha n\rfloor}\right\|_{\square}\right)
$$

goes to zero exponentially; that is, there are $C_{3}, \Lambda_{3} \in \mathbb{R}^{+}, \Lambda_{3}<1$ such that

$$
C \Lambda^{n}\left(\left\|\pi\left(f \circ F^{\lfloor\alpha n\rfloor}\right)\right\|_{-}+\left\|f \circ F^{\lfloor\alpha n\rfloor}\right\|_{\square}\right) \leq C_{3} \Lambda_{3}^{n}\left(\|\pi(f)\|_{-}+\|f\|_{\square}+\|f\|_{\uparrow l i p}\right) .
$$

Let us evaluate each term of the above sum. We recall the definition of $\operatorname{Lip}_{y}(g)$ from (2.4). We observe that we can bound the first summand in the above inequality in the following way

$$
C \Lambda^{k}\left(\|\pi(1)\|_{-}+\|1\|_{\infty}\right)\left\|f \cdot\left(g \circ F^{n}\right)\right\|_{\uparrow l i p} \leq C_{2} \Lambda^{\lfloor\alpha n\rfloor}\left(\left\|f \cdot\left(g \circ F^{n}\right)\right\|_{\infty !}+\operatorname{Lip}_{y}\left(f \cdot\left(g \circ F^{n}\right)\right)\right) .
$$

Since $F$ is contracting on the vertical direction, when $n$ grows we get

$$
\operatorname{Lip}_{y}\left(g \circ F^{n}\right) \rightarrow 0, \quad \operatorname{Lip}_{y}\left(g \circ F^{n}\right) \leq \operatorname{Lip}_{y}(g) \text { and } \quad\left\|g \circ F^{n}\right\|_{\uparrow l i p} \leq\|g\|_{\uparrow l i p} \text {. }
$$

Then, for all big enough $n, C_{2} \Lambda^{\lfloor\alpha n\rfloor}\left(\left\|f \cdot\left(g \circ F^{n}\right)\right\|_{\infty !}+\operatorname{Lip}_{y}\left(f \cdot\left(g \circ F^{n}\right)\right)\right)$ is bounded from above by

$$
C_{2} \Lambda^{\lfloor\alpha n\rfloor}\left(\|g\|_{\infty !}\|f\|_{\infty !}+\|g\|_{\infty !} \operatorname{Lip}_{y}(f)+\|f\|_{\infty !} \operatorname{Lip}_{y}(g)\right) \leq C_{2} \Lambda^{\lfloor\alpha n\rfloor}\|g\|_{\uparrow l i p}\|f\|_{\uparrow l i p} .
$$

The second summand can be estimated as

$$
\begin{aligned}
& C \Lambda^{n}\left(\left\|\pi\left(f \circ F^{k}\right)\right\|_{-}+\left\|f \circ F^{k}\right\|_{\infty}\right) \cdot\left\|g \circ F^{k}\right\|_{\uparrow l i p} \\
& \quad \leq C \Lambda^{n}\left(\left\|\pi\left(f \circ F^{\lfloor\alpha n\rfloor}\right)\right\|_{-}+\left\|f \circ F^{\lfloor\alpha n\rfloor}\right\|_{\infty}+\left\|f \circ F^{\lfloor\alpha n\rfloor}\right\|_{\square}\right)\left\|g \circ F^{\lfloor\alpha n\rfloor}\right\|_{\uparrow l i p} \\
& \quad \leq\left(C_{3} \Lambda_{3}^{n}\left(\|\pi(f)\|_{-}+\|f\|_{\uparrow l i p}+\|f\|_{\square}\right)+C \Lambda^{n}\|f\|_{\infty}\right)\|g\|_{\uparrow l i p},
\end{aligned}
$$

where the last inequality is obtained using inequality (2.9). Finally, the last term is

$$
C \Lambda^{\lfloor\alpha n\rfloor}\left|\int g d \mu\right|\|f\|_{\uparrow l i p}\left(\|\pi(1)\|_{-}+\|1\|_{\infty}\right),
$$

Altogether, we can bound $\left|\int g \circ F^{n} f d \mu-\int g d \mu \int f d \mu\right|$ by $(I)+(I I)+(I I I)+(I V)$, where

$$
\begin{aligned}
(I) & =C_{2} \Lambda^{\lfloor\alpha n\rfloor}\|g\|_{\uparrow l i p}\|f\|_{\uparrow l i p} \\
(I I) & =C_{3} \Lambda_{3}^{n}\left(\|\pi(f)\|_{-}+\|f\|_{\uparrow l i p}+\|f\|_{\square}\right)\|g\|_{\uparrow l i p} \\
(I I I) & =C \Lambda^{n}\|f\|_{\infty}\|g\|_{\uparrow l i p} \\
(I V) & =C \Lambda^{\lfloor\alpha n\rfloor}\left|\int g d \mu\right|\|f\|_{\uparrow l i p}\left(\|\pi(1)\|_{-}+\|1\|_{\infty}\right)
\end{aligned}
$$

which finally gives

$$
\left|\int g \circ F^{n} f d \mu-\int g d \mu \int f d \mu\right| \leq C_{4} \Lambda_{4}^{n}\|g\|_{\uparrow l i p}\left(\|f\|_{\uparrow l i p}+\|\pi(f)\|_{-}+\|f\|_{\square}\right) .
$$

For ease of reference, the previous results can be summarized as follows.

Theorem 3. Let $F: Q \circlearrowleft$ be a Borel function such that $F(x, y)=(T(x), G(x, y))$, $\mu$ an $F$ invariant probability measure with $T$-invariant marginal $\mu_{x}$ on the $x$-axis and satisfying 
(1) $\left(T, \mu_{x}\right)$ has exponential convergence to equilibrium with respect to the norm $\|\cdot\|_{1}$ and to a norm $\|\cdot\|_{-}$;

(2) $T$ is nonsingular with respect to the Lesbegue measure, piecewise continuous and monotonic: there is a collection of intervals $\left\{I_{i}\right\}_{i=1, \ldots, m}, \cup I_{i}=I$ such that on each $I_{i}, T$ is an homeomorphism onto its image.

(3) $F$ is a uniform contraction on each vertical leaf.

Moreover, let us assume that that there are $C, K \in \mathbb{R}$ and a seminorm $\|\cdot\|_{\square}$ such that

$$
\left\|\pi\left(f \circ F^{n}\right)\right\|_{-}+\left\|f \circ F^{n}\right\|_{\square} \leq C_{1} K^{n}\left(\|\pi(f)\|_{-}+\|f\|_{\uparrow l i p}+\|f\|_{\square}\right), \quad n \geq 1 .
$$

Then $F$ has exponential decay of correlations: there are $C, \Lambda \in \mathbb{R}^{+}, \Lambda<1$

$$
\left|\int f \cdot\left(g \circ F^{n}\right) d \mu-\int g d \mu \int f d \mu\right| \leq C_{2} \Lambda^{n}\|g\|_{\uparrow l i p}\left(\|f\|_{\uparrow l i p}+\|\pi(f)\|_{-}+\|f\|_{\square}\right)
$$

for all $f, g: Q \rightarrow \mathbb{R}$ and $n \geq 0$.

To use this result in concrete examples we must find suitable norms $\|\cdot\|_{-}$and $\|\cdot\|_{\square}$ satisfying the above assumptions in each particular application. For the case of Poincaré maps of singular hyperbolic systems such norms will be introduced in the next section.

\section{3. $p$-BOUNDED VARIATION AND $C^{1+\alpha}$ PIECEWISE EXPANDING MAPS}

We recall the main definitions and results about $p$-bounded variation and iteration of piecewise expanding maps $T$ such that $\frac{1}{T^{r}}$ has $p$-bounded variation. Almost everything in this section is taken from [28. We will however put the results of [28] in a form which can be used for our purposes.

Given a function $g:[0,1] \rightarrow \mathbb{R}$ we define its universal $p$-variation as the following adaptation of the usual notion of bounded variation:

$$
\begin{aligned}
\operatorname{var}_{p}\left(g, x_{1}, \ldots, x_{n}\right) & =\left(\sum_{i \leq n}\left|g\left(x_{i}\right)-g\left(x_{i+1}\right)\right|^{p}\right)^{\frac{1}{p}} \\
\operatorname{var}_{p}(g) & =\sup _{\left(x_{i}\right) \in \text { Finite subdivisions of }[0,1]} \operatorname{var}_{p}\left(g, x_{1}, \ldots, x_{n}\right) .
\end{aligned}
$$

Let us also define $U B V_{p}=\left\{g: \operatorname{var}_{p}(g)<\infty\right\}$.

We will need another definition of variation for maps with two variables that we present here for convenience. Similarly to the one dimensional case, if $f: Q \rightarrow \mathbb{R}$ and $x_{i} \leq x_{2} \leq \ldots \leq x_{n}$, let us define

$$
\operatorname{var}^{\square}\left(f, x_{1}, \ldots, x_{n}, y_{1}, \ldots, y_{n}\right)=\sum_{1 \leq i \leq n}\left|f\left(x_{i}, y_{i}\right)-f\left(x_{i+1}, y_{i}\right)\right| .
$$

We then consider the supremum $\operatorname{var}^{\square}\left(f, x_{1}, \ldots, x_{n}, y_{1}, \ldots, y_{n}\right)$ over all subdivisions $x_{i}$ and all choices of the $y_{i}$

$$
\operatorname{var}^{\square}(f)=\sup _{n}\left(\sup _{\left(x_{i} \leq x_{2} \leq \ldots \leq x_{n}\right) \in \mathbb{I},\left(y_{i}\right) \in \mathbb{I}} \operatorname{var}^{\square}\left(f, x_{1}, \ldots, x_{n}, y_{1}, \ldots, y_{n}\right)\right) .
$$

Let $m$ be the Lebesgue measure on the unit interval, $\varepsilon>0$ and $h: \mathbb{I} \rightarrow \mathbb{C}$. We define

$$
\operatorname{osc}(h, \varepsilon, x)=\operatorname{ess} \sup \left\{\left|h\left(y_{1}\right)-h\left(y_{2}\right)\right|: y_{1}, y_{2} \in B_{\varepsilon}(x) \cap \mathbb{I}\right\},
$$

where $B_{\varepsilon}(x)$ is the ball centered in $x$ with radius $\varepsilon$, and the essential supremum is taken with respect to the product measure $m^{2}$ on $Q$. Now let us define

$$
\operatorname{osc}_{p}(h, \varepsilon)=\|\operatorname{osc}(h, \varepsilon, x)\|_{p}, \quad 1 \leq p \leq \infty,
$$

where the $p$-norm is taken with respect to $m$.

Remark 7. $\operatorname{osc}_{p}(h, *):(0, A] \rightarrow[0, \infty]$ is a non decreasing function and $\operatorname{osc}_{p}(h, \varepsilon) \geq \operatorname{osc}_{1}(h, \varepsilon)$.

Fixed $0 \leq r \leq 1$, set $R_{p, r, n}=\left\{h \mid \forall \varepsilon \in(0, A], \operatorname{osc}_{p}(h, \varepsilon) \leq n \varepsilon^{r}\right\}$ and $S_{p, r}=\cup_{n \in \mathbb{N}} R_{p, r, n}$.

We can now define: 
(1) $B V_{p, r}$ as the space of $m$-equivalence classes of functions in $S_{p, r}$

(2) $\operatorname{var}_{p, r}(h)=\sup _{0<\varepsilon<A}\left(\varepsilon^{-r} \operatorname{osc}_{p}(h, \varepsilon)\right)$

(we remark that this definition depends on a fixed constant $A$ and that $\operatorname{var}_{p, r}(h) \geq$ $\left.\operatorname{var}_{1, r}(h)\right)$.

(3) for $h \in B V_{p, r}$ we define $\|h\|_{p, r}:=\operatorname{var}_{p, r}(h)+\|h\|_{p}$.

It turns out that $B V_{p, r}$ with the norm $\|h\|_{p, r}$ is a Banach space; see [28, Thm. 1.13] and [28, Lemma 2.7].

Proposition 4. $U B V_{p} \subseteq B V_{p, \frac{1}{p}} \subseteq B V_{1, \frac{1}{p}}$ for all $1 \leq p<\infty$. Moreover

$$
\operatorname{var}_{1, \frac{1}{p}}(h) \leq \operatorname{var}_{p, \frac{1}{p}}(h) \leq 2^{\frac{1}{p}} \operatorname{var}_{p}(h) .
$$

In what follows we need to compare the $\|\cdot\|_{p, r}$ norm with the $L^{\infty}(m)$ norm.

Lemma 2. If $f \in B V_{1, r}(r \leq 1)$, then $f \in L^{\infty}(m)$ and $\|f\|_{\infty} \leq A^{r-1} \cdot\|f\|_{1, r}$, where $A$ is the constant in the definition of $\|\cdot\|_{1, r}$ (see item 2 above) and $\|\cdot\|_{\infty}$ is the norm of $L^{\infty}(m)$.

Proof. From the definition of $\operatorname{var}_{p, r}(f)$ and considering $\varepsilon=A$, then $\operatorname{var}_{1, r}(f) \geq A^{-r} \cdot \operatorname{osc}_{1}(f, A)$ and $\int_{[0,1]}|\operatorname{osc}(f, A, x)| d m(x) \leq A^{r} \cdot \operatorname{var}_{1, r}(f)$.

Moreover $B_{\varepsilon}(x) \supseteq B_{\varepsilon / 2}(y)$ implies $\operatorname{osc}(f, \varepsilon, x) \geq \operatorname{osc}(f, \varepsilon / 2, y)$, and then

$$
\int_{[0, A]}|\operatorname{osc}(f, A, x)| d m(x) \geq A \cdot \operatorname{osc}(f, A / 2, A / 2),
$$

and by induction we can prove that (where $[x]$ is the biggest integer no larger than $x$ )

$$
\int_{[0,1]}|\operatorname{osc}(f, A, x)| d m(x) \geq A \sum_{i=0}^{[1 / A]+1} \operatorname{osc}\left(f, \frac{A}{2}, \frac{A}{2}+i A\right)
$$

and $\sum_{i=0}^{[1 / A]+1} \operatorname{osc}\left(h, \frac{A}{2}+i A, \frac{A}{2}\right) \geq \operatorname{osc}\left(f, \frac{1}{2}, \frac{1}{2}\right)$ by the triangle inequality. Thus

$$
\operatorname{var}_{1, r}(f) \geq A^{1-r} \operatorname{osc}\left(h, \frac{1}{2}, \frac{1}{2}\right) \text {. }
$$

Finally $\|f\|_{\infty} \leq \operatorname{osc}\left(h, \frac{1}{2}, \frac{1}{2}\right)+\|f\|_{1}$ and then $\|h\|_{1, r}=\operatorname{var}_{1, r}(f)+\|f\|_{1} \geq A^{1-r} \operatorname{osc}\left(h, \frac{1}{2}, \frac{1}{2}\right)+\|f\|_{1} \geq$ $A^{1-r}\|f\|_{\infty}$.

Let us consider the iteration of piecewise expanding maps whose inverse of the derivative has $p$-bounded variation. Let $\left\{I_{1}, \ldots, I_{n}\right\}$ a finite interval partition of $\mathbb{I}$ and $T: \mathbb{I} \circlearrowleft$ be a transformation which is monotone and continuous on each interval $I_{i}$. We assume that

(1) $T$ is nonsingular with respect to the measure $m$;

(2) $T^{\prime}$ exists inside the intervals and $\frac{1}{T^{\prime}}$ is bounded almost everywhere;

Under these assumptions let us consider a measurable and bounded function $f$ and the PerronFrobenius operator related to $T$

$$
P f(x)=\sum_{i=1}^{N} \frac{f}{T^{\prime}} \circ T_{i}^{-1} \cdot 1_{T\left(I_{i}\right)}, \text { where } T_{i}=\left.T\right|_{I_{i}} .
$$

This operator represent the action of the transfer operator on densities of absolutely continuous measures, and extends to a linear contraction on $L^{1}(m)$. Under these assumptions, if the map $T$ is piecewise expanding, and $1 /\left|T^{\prime}\right|$ has universal $p$-bounded variation, a kind of Lasota Yorke inequality can be proved (see [28, Thm 3.2 and 3.5]).

Theorem 4 (Lasota Yorke inequality for $P$ ). Let T, $P, m$ be as described above.

(1) If $\frac{1}{T^{\prime}} \in U B V_{p}$ (where $1 \leq p<\infty$ ) and

(2) there is an $n \in \mathbb{N}$ with $\left\|\frac{1}{\left(T^{n}\right)^{\prime}}\right\|_{\infty}<1$,

then there are $0<\beta<1$ and $C>0$ such that for each $f \in B V_{1, \frac{1}{p}}$

$$
\|P f\|_{1, \frac{1}{p}} \leq \beta\|f\|_{1, \frac{1}{p}}+C\|f\|_{1} \text {. }
$$


Since $P$ is a $L^{1}(m)$-contraction, the theorem of Ionescu-Tulcea-Marinescu [27] provides a description of the spectral properties of $P$; see [28, Thm. 3.3].

(1) $P: L^{1}(m) \rightarrow L^{1}(m)$ has a finite number of eingenvalues $\lambda_{1}, \ldots, \lambda_{r}$ of modulus 1 ;

(2) Let $E_{i}=\left\{f \in L^{1}(m) \mid P f=\lambda_{i} f\right\}$, then $E_{i} \subset B V_{1, \frac{1}{p}}$ and $\operatorname{dim}\left(E_{i}\right)<\infty(i=1, \ldots, r)$;

(3) $P=\sum_{i=1}^{r} \lambda_{i} \Psi_{i}+Q$, where $\Psi_{i}$ are projections onto the eigenspaces $E_{i}$ with $\left\|\Psi_{i}\right\|_{1} \leq 1$ and $Q$ is a linear operator on $L^{1}(m)$ with $Q\left(B V_{1, \frac{1}{p}}\right) \subseteq B V_{1, \frac{1}{p}}, \sup _{n}\left\|Q^{n}\right\|_{1}<\infty$ and $\left\|Q^{n}\right\|_{1, \frac{1}{p}}=O\left(\Lambda^{n}\right)$ for some $0<\Lambda<1$. Furthermore $\Psi_{i} \Psi_{j}=0(i \neq j)$ and $\Psi_{i} Q=Q \Psi_{i}=0$ (for all $i$ );

(4) 1 is an eigenvalue of $P$, and assuming $\lambda_{1}=1$ and $h=\Psi_{1}(1), \mu=h m$ is the greatest $T$ invariant probability on $X$ absolutely continuous with respect to $m$, i.e., if $\tilde{\mu}$ is $T$-invariant and $\tilde{\mu}<<m$, then $\tilde{\mu}<<\mu$;

(5) there is a finite partition $\left\{C_{l, k}\right\}_{l=1, \ldots, r ; k=1, \ldots, L_{l}}$ of $[0,1]$ such that $T\left(C_{l, k}\right)=C_{l, k+1}$ for $k=1, \ldots, L_{1}-1$ and $T\left(C_{l, L_{1}}\right)=C_{l, 1}$; moreover, $\left.T^{L_{l}}\right|_{C_{l, k}}$ is weakly mixing for each $k, l$.

Remark 8. The above result (item (5)) tells that some iterate $P^{n}$ of the transfer operator has a finite set of a.c.i.m. with no eigenvalues other than 1 on the unit circle. An iterated of the system can be hence decomposed into a finite union of invariant sets and each invarant subsystem has a unique a.c.i.m and no other eigenvalues on the unit circle.

This implies exponential convergence to equilibrium for some iterate of the map, as needed in Theorem 2 .

Proposition 5. Under the same assumptions as above, if $g \in L^{1}(m), f \in B V_{1, \frac{1}{p}}$, $\mu$ is an a.c.i.m., the associated transfer operator has 1 as a simple eigenvalue and there are no more eigenvalues on the unit circle, then there is $C, \Lambda>0, \Lambda \leq 1$ s.t.

$$
\left|\int g\left(T^{n}(x)\right) f(x) d m-\int g(x) d \mu \int f(x) d m\right| \leq C \Lambda^{n} \cdot\|g\|_{1} \cdot\|f\|_{1, r} .
$$

Proof. Let us set $f_{0}=\Psi_{1}(1)$. Since 1 is a single eigenvalue and there are no more eigenvalues on the unit circle, then $B V_{1, \frac{1}{p}}=\mathbb{R} f_{0}+X_{0}$ (this decomposition is invariant by $P$ ), and $\operatorname{spec}\left(P \mid X_{0}\right)$ is contained in a disc with radius $\Lambda<1$. Without loss of generality we can suppose that $\int f(x) d m=$ 1. By item (4) above, $f_{0}$ is the density of the invariant measure $\mu$, therefore

$$
\begin{aligned}
\int g\left(T^{n}(x)\right) f(x) d m-\int g(x) d \mu \int f(x) d m & = \\
\int g \cdot P^{n} f d m-\int g \cdot f_{0} d m & =\int g \cdot\left(P^{n} f-f_{0}\right) d m \\
\int g \cdot\left(P^{n}\left(f-f_{0}\right)\right) d m & =\int g \cdot\left(P^{n}\left(\pi_{0} f\right)\right) d m
\end{aligned}
$$

where $\pi_{0}(g)=g-f_{0} \int g d m$ is the spectral projection onto $X_{0}=\left\{f: \int f d m=0\right\}$. Then, by Lemma 2 and the spectral radius theorem, we get the needed estimation

$$
\begin{aligned}
\left|\int g \cdot\left(P^{n}\left(\pi_{0} f\right)\right) d m\right| & \leq\|g\|_{1}\left\|P^{n}\left(\pi_{0} f\right)\right\|_{\infty} \leq\|g\|_{1} A^{r-1}\left\|P^{n}\left(\pi_{0} f\right)\right\|_{1, r} \\
& \leq C^{\prime} \Lambda^{n}\|g\|_{1}\left\|\pi_{0} f\right\|_{1, r} \leq C^{\prime \prime} \Lambda^{n}\|g\|_{1}\|f\|_{1, r} .
\end{aligned}
$$

\section{Singular-HYPERBolic ATtraCtors}

In this section we will introduce what nowadays is called a singular-hyperbolic attractor for a 3-dimensional vector field $X$ (or flow $X_{t}$ ).

The singular-hyperbolic class of attractors we consider contains the partially hyperbolic attractors $\Lambda$ of a $C^{2}$ vector field $X$, with finitely many equilibria accumulated by regular orbits of $X$ in $\Lambda$, and admitting a continuous and $D X_{t}$-invariant splitting of the tangent bundle over $\Lambda$ into a pair $T_{\Lambda} M=E_{\Lambda}^{s} \oplus E_{\Lambda}^{c u}$ of vector sub-bundles. Here $E_{\Lambda}^{s}$ has one-dimensional fibers and is uniformly 
contracted by $D X_{t}$, and $E_{\Lambda}^{c u}$ has two-dimensional fibers whose area is uniformly expanded by $D X_{t}$; see below for precise definitions. As shown in [35, this class is an extension of the class of uniformly hyperbolic attractors, since every singular-hyperbolic attractor containing no equilibria of $X$ is uniformly hyperbolic, that is, the sub-bundle $E_{\Lambda}^{c u}$ admits a further continuous and $D X_{t^{-}}$ invariant splitting $E_{\Lambda}^{c u}=E_{\Lambda}^{X} \oplus E_{\Lambda}^{u}$ into the line bundle generated by the flow direction over $\Lambda$, and the bundle $E_{\Lambda}^{u}$ with one-dimensional fibers uniformly expanded by $D X_{t}$. In particular, Anosov (or globally hyperbolic) flows on three-dimensional manifolds belong to this class. Moreover, and more important, the class of singular-hyperbolic attractors contains every $C^{1}$ robustly transitive isolated set for flows on compact three-manifolds $M$. That is, as proved in [35], if for a given open subset $U$ of $M$ there exists a $C^{1}$ open subset $\mathcal{U}$ of $\mathfrak{X}^{1}(M)$ (the family of $C^{1}$ vector fields of $M$ ) such that the maximal invariant subset $\Lambda_{Y}(U)$ of $U$ contains a non-trivial transitive orbit of $Y$ for every $Y \in \mathcal{U}$, then $\Lambda_{Y}(U)$ is a singular-hyperbolic attractor and contains at most finitely many hyperbolic saddle-type equilibria, either for $Y$ of for $-Y$, for each $Y \in \mathcal{U}$.

Next we describe precisely what we mean by a singular-hyperbolic attractor. We start by recalling some definitions and notations and then we shall list some facts about this kind of attractors proved elsewhere but that will be useful for us. We also adapt some known results to our case and add some new ones, to meet the requirements needed to prove the decay of correlations and the logarithm law; the main results proved are listed in Theorem 5 below.

Let $M$ be a 3-dimensional compact riemanian manifold and $\mathcal{X}^{r}(M), r \geq 1$, be set of $C^{r}$ vector fields (or flows) defined on $M$. Given a compact invariant set $\Lambda$ of $X \in \mathcal{X}^{r}(M)$, we say that $\Lambda$ is isolated if there exists an open set $U \supset \Lambda$ such that

$$
\Lambda=\bigcap_{t \in \mathbb{R}} X_{t}(U)
$$

If $U$ above can be chosen such that $X_{t}(U) \subset U$ for $t>0$, we say that $\Lambda$ is an attracting set.

Given $X \in \mathcal{X}^{r}(M)$, a point $x \in M$ is regular if $X(x) \neq 0$. In this case we refer to its orbit as a regular $X$-orbit or regular orbit for short.

Given $p \in M$, we define $\omega_{X}(p)$ as the set of accumulation points of the positive orbit $\left\{X_{t}(p) ; t \geq\right.$ $0\}$ of $p$. We also define $\alpha_{X}(p)=\omega_{-X}(p)$, where $-X$ is the time reversed vector field.

A subset $\Lambda \subset M$ is transitive if it has a full dense orbit, that is, there is $p \in M$ such that $\omega_{X}(p)=\Lambda=\alpha_{X}(p)$.

Definition 3. An attractor is a transitive attracting set, and a repeller is an attractor for the reversed vector field $-X$.

An attractor, or repeller, is proper if it is not the whole manifold. An invariant set of $X$ is non-trivial if it is neither a periodic orbit nor an equilibrium of $X$. Recall that a point $\sigma \in M$ is an equilibrium of $X$ if $X(\sigma)=0$.

Definition 4. Let $\Lambda$ be a compact invariant set of $X \in \mathcal{X}^{r}(M), c>0$, and $0<\lambda<1$. We say that $\Lambda$ has a $(c, \lambda)$-dominated splitting if the bundle over $\Lambda$ can be written as a continuous $D X_{t}$-invariant sum of sub-bundles

$$
T_{\Lambda} M=E^{1} \oplus E^{2},
$$

such that for every $t>0$ and every $x \in \Lambda$, we have

$$
\left\|D X_{t}\left|E_{x}^{1}\|\cdot\| D X_{-t}\right| E_{X_{t}(x)}^{2}\right\|<c \cdot \lambda^{t} .
$$

We say that $\Lambda$ is partially hyperbolic if it has a $(c, \lambda)$-dominated splitting such that the sub-bundle $E^{1}$ is uniformly contracting, that is, for some $c>0$ and every $t>0$ and each $x \in \Lambda$ we have

$$
\left\|D X_{t} \mid E_{x}^{1}\right\|<c \lambda^{t} .
$$

In this case we denote the one-dimensional bundle $E^{1}$ by $E^{s}$ and the two-dimensional bundle $E^{2}$ by $E^{c u}$. We refer to $E^{s}$ as the contracting direction and to $E^{c u}$ as the central or center-unstable direction of the splitting.

The next proposition is an immediate consequence of [21, Theorem 1] and will simplify many of the arguments. 
Proposition 6. There exists an adapted Riemannian metric $\|\cdot\|_{0}$, equivalent to the original one, and $\lambda \in(0,1)$ such that

$$
\begin{array}{r}
\left\|D X_{t}\left|E_{x}^{1}\left\|_{0} \cdot\right\| D X_{-t}\right| E_{X_{t}(x)}^{2}\right\|_{0}<\lambda^{t}, \quad \text { and } \\
\left\|D X_{t} \mid E_{x}^{1}\right\|<\lambda^{t},
\end{array}
$$

for all $t \geq 0$, and all $x \in \Lambda$.

Throughout the remaining of the paper we assume that the Riemannian metric is an adapted metric and the first sub-bundle $E^{1}$ is one-dimensional. For simplicity we denote the adapted metric by $\|\cdot\|$.

For $x \in \Lambda$ and $t \in \mathbb{R}$ we let $J_{t}^{c u}(x)$ be the absolute value of the determinant of the linear map

$$
D X_{t} \mid E_{x}^{c u}: E_{x}^{c u} \rightarrow E_{X_{t}(x)}^{c u}
$$

We say that the sub-bundle $E_{\Lambda}^{c u}$ of the partially hyperbolic invariant set $\Lambda$ is volume expanding if

$$
J_{t}^{c u}(x) \geq K e^{\theta t} \quad \text { for every } x \in \Lambda \text { and } t \geq 0 \text { and some } \theta>0 .
$$

In this case we say that $E_{\Lambda}^{c u}$ is $(K, \theta)$-volume expanding to indicate the dependence on $K, \theta$. This condition is weaker than the uniform exponential expansion along the central direction. The Geometric Lorenz attractor has a volume expanding central direction that is not uniformly expanding [6].

The domination condition (4.1), together with the volume expanding condition (4.5) along the central direction, imply that the direction of the flow is contained in the central bundle $E^{c u}$, Lemma 6.1, pg 163].

Recall that an equilibrium $\sigma$ of $X$ is hyperbolic only if the real part of every eigenvalue of $D X(\sigma)$ is distinct of zero, see [6. Section 2.1,pp 6]. Recall also the definition of a special type of equilibrium of a vector field $X$ in a 3 -manifold.

Definition 5. We say that an equilibrium $\sigma$ of a 3-vector field $X$ is Lorenz-like if the eigenvalues $\lambda_{i}, 1 \leq i \leq 3$, of $D X(\sigma)$ are real and satisfy

$$
\lambda_{2}<\lambda_{3}<0<-\lambda_{3}<\lambda_{1} .
$$

If $\sigma$ is a Lorenz-like equilibrium, letting $\alpha=-\frac{\lambda_{3}}{\lambda_{1}}$ and $\beta=-\frac{\lambda_{2}}{\lambda_{1}}$ we obtain $0<\alpha<1<\beta$.

Definition 6 (Singular Hyperbolic Attractor). Let $\Lambda$ be a compact invariant set of $X \in \mathcal{X}^{r}(M)$. We say that $\Lambda$ is a singular-hyperbolic set for $X$ if all the equilibria of $\Lambda$ are hyperbolic, and $\Lambda$ is partially hyperbolic with volume expanding central direction. A singular-hyperbolic set which is also an attractor will be called a singular-hyperbolic attractor.

The next result is the content of [6, Lemma 5.27]:

Lemma 3. Let $\Lambda$ be a singular-hyperbolic attractor of a 3-dimensional vector field $X$. Then, every equilibrium $\sigma$ properly accumulated by regular orbits within $\Lambda$ is Lorenz-like either for $X$ or for $-X$.

An isolated set $\Lambda$ of a $C^{1}$ vector field $X$ is robustly transitive if it has an open neighborhood $U$ such that

$$
\Lambda_{Y}(U)=\bigcap_{t \in \mathbb{R}} Y_{t}(U)
$$

is both transitive and non-trivial (i.e., it is neither an equilibrium point nor a periodic orbit) for any vector field $Y C^{1}$-close to $X$. Roughly speaking, $\Lambda$ is robustly transitive if it can not be destroyed by small $C^{1}$ perturbations.

Morales, Pacifico, and Pujals proved in 35] that any transitive robust invariant set of a 3dimensional flow containing some equilibrium is a singular-hyperbolic attractor or repeller. In the absence of equilibria, robustness implies uniform hyperbolicity. The most meaningful examples of singular-hyperbolic attractors are the Lorenz attractor [32] and the so called Geometric Lorenz attractor [2, 22].

The main results we explain and derive in this section that will be used in the rest of the paper are stated in the following. 
Theorem 5. For an open and dense subset of $C^{2}$ vector fields $X$ having a singular hyperbolic attractor $\Lambda$ on a 3-manifold, there exists a finite family $\Xi$ of cross-sections and a global ( $n$-th return) Poincaré map $R: \Xi_{0} \rightarrow \Xi, R(x)=X_{\tau(x)}(x)$ such that

(1) the domain $\Xi_{0}=\Xi \backslash \Gamma$ is the entire cross-sections with a family $\Gamma$ of finitely many smooth arcs removed and $\tau: \Xi_{0} \rightarrow\left[\tau_{0},+\infty\right)$ is a smooth function bounded away from zero by some uniform constant $\tau_{0}>0$.

(2) We can choose coordinates on $\Xi$ so that the map $R$ can be written as $F: \tilde{Q} \rightarrow Q$, $F(x, y)=(T(x), G(x, y))$, where $Q=\mathbb{I} \times \mathbb{I}, \mathbb{I}=[0,1]$ and $\tilde{Q}=Q \backslash \Gamma_{0}$ with $\Gamma_{0}=\mathcal{C} \times \mathbb{I}$ and $\mathcal{C}=\left\{c_{1}, \ldots, c_{n}\right\} \subset \mathbb{I}$ a finite set of points.

(3) The map $T: \mathbb{I} \backslash \mathcal{C} \rightarrow \mathbb{I}$ is $C^{1+\alpha}$ piecewise monotonic with $n+1$ branches defined on the connected components of $\mathbb{I} \backslash \mathcal{C}$ and has a finite set of a.c.i.m., $\mu_{T}^{i}$. Also inf $\left|T^{\prime}\right|>1$ where it is defined, $1 /\left|T^{\prime}\right|$ has universal bounded $p$-variation and then $d \mu_{T}^{i} / d m$ has bounded $p$-variation.

(4) The map $G: \tilde{Q} \rightarrow \mathbb{I}$ preserves and uniformly contracts the vertical foliation $\mathcal{F}=\{\{x\} \times$ $\mathbb{I}\}_{x \in \mathbb{I}}$ of $Q$ : there exists $0<\lambda<1$ such that $\operatorname{dist}\left(G\left(x, y_{1}\right), G\left(x, y_{2}\right)\right) \leq \lambda \cdot\left|y_{1}-y_{2}\right|$ for each $y_{1}, y_{2} \in \mathbb{I}$. In addition, the map $G$ satisfies $\operatorname{var}^{\square}(G)<\infty$.

(5) The map $F$ admits a finite family of physical probability measures $\mu_{F}^{i}$ which are induced by $\mu_{T}^{i}$ in a standard way. The Poincaré time $\tau$ is integrable both with respect to each $\mu_{F}^{i}$ and with respect to the two-dimensional Lebesgue area measure of $Q$.

(6) Moreover if, for all singularities $\sigma \in \Lambda$, we have the eigenvalue relation $-\lambda_{2}(\sigma)>\lambda_{1}(\sigma)$, then the second coordinate map $G$ of $F$ has a bounded partial derivative with respect to the first coordinate, i.e., there exists $C>0$ such that $\left|\partial_{x} G(x, y)\right|<C$ for all $(x, y) \in$ $\left(\mathbb{I} \backslash\left\{c_{1}, \ldots, c_{n}\right\}\right) \times \mathbb{I}$.

4.1. Preliminary results. Next we give the notions and establish notations needed to describe the properties of singular-hyperbolic attractors stated in Theorem 5.

4.1.1. Stable foliations on cross-sections. Hereafter, $\Lambda$ is a singular-hyperbolic attractor of $X \in$ $\mathcal{X}^{2}(M)$ with invariant splitting $T_{\Lambda} M=E^{s} \oplus E^{c u}$ with $\operatorname{dim} E^{c u}=2$. Let $\tilde{E}^{s} \oplus \tilde{E}^{c u}$ be a continuous extension of this splitting to a small neighborhood $U_{0}$ of $\Lambda$. For convenience, we take $U_{0}$ to be forward invariant and such that $\cap_{t \geq 0} X_{t}\left(U_{0}\right)=\Lambda$. We will see in Subsection 4.1.2 that $\tilde{E}^{s}$ may be chosen invariant under the derivative. In general, the extension $\tilde{E}^{c u}$ of the center-unstable direction cannot be assumed to be invariant; see [25] and also [10. Appendix B]. However, we can always consider a cone field around it on $U_{0}$

$$
C_{a}^{c u}(x)=\left\{v=v^{s}+v^{c u}: v^{s} \in \tilde{E}_{x}^{s} \text { and } v^{c u} \in \tilde{E}_{x}^{c u} \text { with }\left\|v^{s}\right\| \leq a \cdot\left\|v^{c u}\right\|\right\}
$$

which is forward invariant for some $a>0$, that is, there is a large $T>0$ depending on $a$, but not depending on $x \in U_{0}$, such that

$$
D X_{t}\left(C_{a}^{c u}(x)\right) \subset C_{a}^{c u}\left(X_{t}(x)\right) \text { for all } t \geq T .
$$

Moreover, we may take $a>0$ arbitrarily small, reducing $U_{0}$ if necessary. For notational simplicity, we write $E^{s}$ and $E^{c u}$ for $\tilde{E}^{s}$ and $\tilde{E}^{c u}$ in all that follows.

Next let us recall a few classical facts about partially hyperbolic systems, especially existence of stable and strong-stable foliations, and center-unstable foliations. The standard reference are [25, Theorems 4.1, 5.1 and 5.5] and [45, Theorem IV.1].

Before stating the appropriate result we need to review some terms in [25]; namely, the notion of immediate relative $\rho$ pseudo-hyperbolic splitting. To define this, recall that if $L: \mathbb{R}^{d} \rightarrow \mathbb{R}^{d}$ is an injective linear map and $G \subset \mathbb{R}^{d}$ is a subspace, then the conorm of $L$ restricted to $G$ is

$$
m(L \mid G):=\inf _{v \neq 0, v \in G} \frac{\|L v\|}{\|v\|} .
$$

The conorm gives the minimal expansion of $L$ on $G$.

Let $\left\{X_{t}: M \rightarrow M\right\}_{t \in \mathbb{R}}$ be a flow and $\Omega$ be an $X$-invariant compact set. A splitting $T_{\Omega}(M)=$ $E \oplus F$ is immediate relatively $\rho$ pseudo-hyperbolic relative to $X$ if there exists a continuous function 
$\rho: \Omega \rightarrow \mathbb{R}^{+}$such that

$$
\left\|\left.D X_{1}\right|_{E(x)}\right\|<\rho(x)<m\left(\left.D X_{-1}\right|_{F(x)}\right) \quad \text { for all } \quad x \in \Omega .
$$

Using Proposition 6 we have the following

Lemma 4. Let $X$ be a $C^{2}$ flow and $\Lambda$ be a singular-hyperbolic attractor with splitting $E^{s} \oplus E^{c u}$. Then $E^{s} \oplus E^{c u}$ is an immediate relatively $\rho$ pseudo-hyperbolic splitting over $\Lambda$ relative to $X$ for some continuous function $\rho: \Lambda \rightarrow \mathbb{R}^{+}$.

Proof. The domination assumption ensures that $\left\|\left.D X_{1}\right|_{E(x)}\right\|<\lambda m\left(\left.D X_{-1}\right|_{F\left(X_{1}(x)\right)}\right)$ for all $x \in \Lambda$, so

$$
m\left(\left.D X_{-1}\right|_{F\left(X_{1}(x)\right)}\right)>\frac{\left\|\left.D X_{1}\right|_{E(x)}\right\|}{\lambda}:=\rho(x)>\left\|\left.D X_{1}\right|_{E(x)}\right\|
$$

and the above function $\rho: \Lambda \rightarrow \mathbb{R}^{+}$is continuous since a dominated splitting is continuous.

We then have the following restatement of Theorems [45, Theorem IV.1] and 25, Theorem 5.5]:

Theorem 6. Let $X$ be a $C^{2}$ flow and $\Lambda$ be a compact $X$-invariant singular-hyperbolic attractor $\Lambda$ having a dominated splitting $E^{s} \oplus E^{c u}$. There are $\varepsilon>0$ and $\lambda \in(0,1)$ such that for every point $x \in \Lambda$ there are two embedded discs $W_{\varepsilon}^{s s}(x)$ and $W_{\varepsilon}^{c u}(x)$ tangent at $x$ to $E^{s}(x)$ and $E^{c u}(x)$ respectively, and satisfying, for all $t>0$

(1) $W_{\varepsilon}^{s s}(x)$ is a $C^{2}$ embedded disc,

(2) $W_{\varepsilon}^{s s}(x)=\left\{q \in M ; \operatorname{dist}\left(X_{t}(x), X_{t}(q)\right) \leq \varepsilon\right.$ and $\left.\lim _{t \rightarrow \infty} \operatorname{dist}\left(X_{t}(x), X_{t}(q)\right) / \lambda^{t}=0\right\}$,

(3) $X_{t}\left(W_{\varepsilon}^{s s}(x)\right) \subset W_{\varepsilon}^{s s}\left(X_{t}(x)\right)$,

(4) The embedding $W_{\varepsilon}^{s s}(x)$ depends $C^{2}$ smoothly on $x$ in the following sense: there is a neighborhood $\mathcal{V}$ of $x$ in $\Lambda$ and a continuous map $\gamma: \mathcal{V} \cap \Lambda \rightarrow \mathrm{Emb}^{2}(\mathbb{I}, M)$ such that $\gamma(x)(0)=x$ and $\gamma(x)(\mathbb{I})=W_{\varepsilon}^{\text {ss }}(x)$, where $\mathrm{Emb}^{2}(\mathbb{I}, M)$ is the collection of all embeddings $\phi: \mathbb{I} \rightarrow M$ endowed with the $C^{2}$ distance;

(5) $X_{t}\left(W_{\varepsilon}^{c u}(x)\right) \cap B_{\varepsilon}(x) \subset W_{\varepsilon}^{c u}\left(X_{t}(x)\right)$, where $B_{\varepsilon}(x)=\{y \in M$; $\operatorname{dist}(x, y)<\varepsilon\}$,

(6) The $W_{\varepsilon}^{c u}(x)$ depend $C^{2}$ smoothly on $x$ as in (4). That is, there exists a continuous map $\gamma$ : $\mathcal{V} \cap \Lambda \rightarrow \operatorname{Emb}^{2}(\mathbb{D}, M)$ such that $\gamma(x)(0)=x$ and $\gamma(x)(\mathbb{D})=W_{\varepsilon}^{c u}(x)$, where $\operatorname{Emb}^{2}(\mathbb{D}, M)$ is the collection of all embeddings $\phi: \mathbb{D} \rightarrow M$ from the unit 2-disk $\mathbb{D}$ into $M$ endowed with the $C^{2}$ distance.

Note that, for $x \in \Lambda, W_{\varepsilon}^{s s}(x)$ and $W_{\varepsilon}^{c u}(x)$ are embedded discs, and so, sub-manifolds of $M$. We refer to $W_{\varepsilon}^{s s}(x)$ as local strong-stable manifold and to $W_{\varepsilon}^{c u}(x)$ as local center-unstable manifold. Since $E^{s}$ is uniformly contracting we have that $W_{\varepsilon}^{s s}(x)$ is uniquely defined. But we stress that the center-unstable $W_{\varepsilon}^{c u}(x)$ manifold is not unique without further assumptions; see [1].

The set

$$
W^{s}(x)=\bigcup_{t \in \mathbb{R}} W_{\varepsilon}^{s s}\left(X_{t}(x)\right) \subset \bigcup_{t \in \mathbb{R}} X_{t}\left(W_{\varepsilon}^{s s}(x)\right)
$$

is called the stable manifold at $x \in \Lambda$. The proof that $W^{s}(x)$ is a manifold is contained in [25, Theorem 5.5].

Denoting $E_{x}^{c s}=E_{x}^{s} \oplus E_{x}^{X}$, where $E_{x}^{X}$ is the direction of the flow at $x$, it follows that

$$
T_{x} W^{s}(x)=E_{x}^{c s} .
$$

4.1.2. Extension of the lamination $\left\{W_{\varepsilon}^{s s}\right\}_{x \in \Lambda}$ to a contracting invariant foliation in a neighborhood of $\Lambda$. Here we show that the collection of $C^{2}$ strong-stable leaves through the points of $\Lambda$, depending continuously on the base point, which is known as a lamination, can be extended to an invariant foliation (in the usual sense from Differential Topology) of a open neighborhood of $\Lambda$, whose leaves are $C^{2}$ submanifolds and whose foliated charts are of class $C^{1}$. In addition, these leaves are uniformly contracted by the action of the flow. The argument we present below follows [37. Appendix 1] closely. 
Let us fix a neighborhood $U_{0}$ of the singular-hyperbolic attractor $\Lambda$ on the manifold $M$ such that the closure of $X_{t}\left(U_{0}\right)$ is contained in $U_{0}$ for all $t>0$. We consider the following family of directions through the points of $U_{0}$

$$
D=\left\{(x, \ell): x \in U_{0}, \ell \in \mathbb{P}^{1}\left(T_{x} M\right)\right\} .
$$

This set is clearly a smooth manifold of dimension 5 . The time-one map of the flow $f=X_{1}$ induces a map $\psi: D \rightarrow \psi(D)$ given by

$$
\psi(x, \ell)=(f(x), D f(x) \cdot \ell), \quad(x, \ell) \in D .
$$

Naturally, this map $\psi$ is of class $C^{1}$ if $f$ is of class $C^{2}$. We note that the subset $\Omega:=\{(x, \ell) \in D$ : $\left.x \in \Lambda, \ell=E_{x}^{s s}\right\}$ is compact and fixed by $\psi$. We claim that it is also a partially hyperbolic subset for $\psi$. To prove this, we consider the inverse map $\varphi(x, \ell)=\left(f^{-1}(x), D f^{-1}(x) \cdot \ell\right)$.

Indeed, for every $(x, \ell) \in \Omega$ we can calculate the derivative $D \varphi$ of $\varphi$ at $(x, \ell) \in \Omega$ using coordinates provided by the splitting of $T_{\Lambda} M$, as follows. Directions on $\mathbb{P}^{1}\left(T_{x} M\right)$ near the stable direction can be seen as graphs of a linear map $\ell: E_{x}^{s s} \rightarrow E_{x}^{c u}$ which in fact is given by the vector $\ell(1) \in E_{x}^{c u}$, since $E_{x}^{s s}$ is one-dimensional. The action of $D f^{-1}(x)$ on the directions of $\mathbb{P}^{1}\left(T_{x} M\right)$ can be represented by the graph transform $\ell^{\prime}=\left.D f^{-1}\right|_{E_{x}^{c u}} \circ \ell \circ\left(\left.D f^{-1}\right|_{E_{x}^{s s}}\right)^{-1}: E_{f^{-1}(x)}^{s s} \rightarrow E_{f^{-1}(x)}^{c u}$. So in this coordinates we have $\varphi(x, \ell)=\left(f^{-1}(x), \ell^{\prime}\right)$. Then we have, since a tangent direction at $\ell \in \mathbb{P}^{1}\left(T_{x} M\right)$ is another linear map $\xi: E_{x}^{s s} \rightarrow E_{x}^{c u}$ given by the vector $\xi(1)$

$$
D \varphi(x, \ell) \cdot(v, \xi)=\left(D f^{-1}(x) \cdot v, D f^{-1}(x) \cdot \xi\right)
$$

which we may represent by the following isomorphism of $\mathbb{R}^{5}$

$$
\left(\begin{array}{cc}
D f^{-1}(x) & 0 \\
0 & \left.\left(\left.D f^{-1}\right|_{E_{x}^{s s}}\right)^{-1} \cdot D f^{-1}\right|_{E_{x}^{c u}}
\end{array}\right):\left(E_{x}^{s s} \oplus E_{x}^{c u}\right) \oplus E_{x}^{c u} \rightarrow\left(E_{f^{-1}(x)}^{s s} \oplus E_{f^{-1}(x)}^{c u}\right) \oplus E_{f^{-1}(x)}^{c u} .
$$

We have now using the domination assumption from (4.3)

$$
\left\|\left.\left(\left.D f^{-1}\right|_{E_{x}^{s s}}\right)^{-1} \cdot D f^{-1}\right|_{E_{x}^{c u}}\right\| \leq\left\|\left.D f\right|_{E_{f-1(x)}^{s s}}\right\| \cdot\left\|\left(\left.D f\right|_{E_{f-1(x)}^{c u}}\right)^{-1}\right\|<\lambda
$$

and by condition (4.2) we get

$$
\left\|\left.\left(\left.D f^{-1}\right|_{E_{x}^{s s}}\right)^{-1} \cdot D f^{-1}\right|_{E_{x}^{c u}}\right\|<\lambda \cdot\left\|\left(\left.D f\right|_{E_{f^{-1}(x)}^{c u}}\right)^{-1}\right\|<m\left(D f^{-1}(x)\right)
$$

since the assumption (4.3) ensures that $\left\|\left(\left.D f\right|_{E_{f^{-1}(x)}^{c u}}\right)^{-1}\right\|<\lambda \cdot\left\|\left.D f\right|_{E_{f^{-1}(x)}^{s s}}\right\|^{-1}$ which is the same as

$$
\left\|\left.D f^{-1}\right|_{E_{x}^{c u}}\right\|<\lambda \cdot\left\|\left.D f^{-1}\right|_{E_{x}^{s s}}\right\|<\left\|\left.D f^{-1}\right|_{E_{x}^{s s}}\right\| .
$$

This shows the partial hyperbolicity of $\Omega$. Hence the map $D \varphi$ is immediate relative $\rho$-pseudohyperbolic for some function $\rho$ strictly smaller than 1 over $\Omega$. We may now use [45, Theorem IV.1] and [25. Theorem 5.5] to obtain a $C^{1}$ center-unstable manifold $W^{c}(x)$ for $\varphi$ through each point $\left(x, E_{x}^{s s}\right) \in \Omega$, which is a center-stable manifold for $\psi$, with dimension 3 and tangent to $T_{x} M \times E_{x}^{s s}$ at $\left(x, E_{x}^{s s}\right)$.

This manifold projects to a neighborhood of $x \in \Lambda$ on $M$ through the canonical projection of $D$ on the first coordinate. This means that on a neighborhood $U \subset U_{0}$ of $\Lambda$ we can define a field of directions $\{\ell(y)\}_{y \in U}$ such that $(y, \ell(y)) \in W^{c}(x)$ for some $x \in \Lambda$. Integrating these direction we obtain $C^{2}$ one-dimensional submanifolds $W^{s s}(y)$ passing through $y \in U$ (note that the field of direction is $C^{1}$ smooth because $W^{c}$ was $C^{1}$ ). Since the lamination $W^{c}$ is $\psi$-invariant, we can deduce that $f\left(W^{s s}(y)\right) \subset W^{s s}(f(y))$ for all $y \in U \cap f^{-1}(U)$.

We have obtained a one-dimensional foliation of a neighborhood $U$ of $\Lambda$ which extends the strong-stable lamination provided by Theorem [6. For small enough $U$ the leaves of this foliation are uniformly contracted by a rate close to $\lambda$ under the action of $f$.

This results extend to the action of $X_{t}$ in a standard way: we have the same conclusions for the diffeomorphism $X_{t}$ for $t>0$ in the place of $f=X_{1}$.

Corollary 3. For any compact invariant subset $\Lambda$ of a $C^{2}$ flow $X_{t}$ which is partially hyperbolic, that is, $\Lambda$ satisfies conditions (4.3) and (4.2) for a continuous $D X_{t}$-invariant splitting $T_{\Lambda} M=$ $E^{s} \oplus E^{c u}$ with one-dimensional stable direction, there exists a neighborhood $U$ of $\Lambda$ in the ambient 
manifold $M$ where a extension $\mathcal{F}^{s}(x)$ of the local stable lamination $\left\{W_{\varepsilon}^{s s}(x)\right\}_{x \in \Lambda}$ is defined, is a locally $X_{t}$-invariant foliation and its leaves are uniformly contracted by $X_{t}$, for all $t>0$. Each leaf is a $C^{2}$ one-dimensional sub-manifold of $M$.

The dependence of $W_{\varepsilon}^{s s}(x)$ on $x$ is similar to item (4) of Theorem 6 but in the $C^{1}$ topology. We stress that the smoothness of $x \mapsto W_{\varepsilon}^{s s}(x)$ is in general not related to the differentiability of the foliation $\mathcal{F}^{s s}=\left\{W_{\varepsilon}^{s s}(x)\right\}_{x \in U}$ of the neighborhood $U$ of $\Lambda$; see e.g. [41.

4.2. Cross-sections and Poincaré maps. Now let $\Sigma$ be a cross-section to the flow, that is, a $C^{2}$ embedded compact disk transverse to $X$ at every point $x \in \Sigma$. We assume from now on that cross-sections are contained in the open neighborhood of $\Lambda$ where a contracting foliation $\mathcal{F}^{s s}$ which extends the strong-stable lamination through the points of $\Lambda$ is defined.

For $x \in \Sigma$ we define $W^{s}(x, \Sigma)$ to be the connected component of $\mathcal{F}^{s c}(x) \cap \Sigma$ that contains $x$, where $\mathcal{F}^{c s}(x):=\cup_{t \in \mathbb{R}} X_{t}\left(\mathcal{F}^{s}(x)\right)$ is the central-stable leaf obtained from the strong-stable leaf $\mathcal{F}^{s}(x)$ in a manner similar to (4.8). Since the flow $\left(X_{t}\right)_{t \in \mathbb{R}}$ is $C^{2}, W^{s}(x, \Sigma)$ is a $C^{2}$ co-dimension one embedded curve for every $x \in \Sigma$. These leaves form a foliation $\mathcal{F}_{\Sigma}^{s}$ of $\Sigma$.

From the celebrated work of Anosov [5] and more recent developments in the partially hyperbolic setting by Pugh-Shub [40] and Brin-Pesin [12, Theorem 3.1], it is known that the holonomies (projection along leaves) between pairs of transverse surfaces to $\mathcal{F}^{s s}$ admit a Hölder Jacobian with respect to Lebesgue induced measure. This naturally implies a similar statement for holonomies transverse to $\mathcal{F}^{c s}$.

In this setting the holonomy (projection) between pairs of transverse curves to $\mathcal{F}_{\Sigma}^{s}$ along the lines of $\mathcal{F}_{\Sigma}^{s}$ can be seen as maps between intervals of the real line having a Hölder Jacobian with respect to Lebesgue measure. Hence these holonomies are $C^{1+\alpha}$ maps, for some $0<\alpha<1$ which depends on $X$ only; see e.g. [6, Section 2.7.2].

In this case the leaves $W^{s}(x, \Sigma)$, for $x \in \Sigma$, define a foliation $\mathcal{F}_{\Sigma}^{s}$ of $\Sigma$ whose transversal smoothness is Hölder- $C^{1}$.

Remark 9. Given a cross-section $\Sigma$ there is no loss of generality in assuming that it is the image of the square $\mathbb{I} \times \mathbb{I}$ by a $C^{1+\alpha}$ diffeomorphism $h$, for some $0<\alpha<1$, which sends vertical lines inside leaves of $\mathcal{F}_{\Sigma}^{s}$. We denote by $\operatorname{int}(\Sigma)$ the image of $(0,1) \times(0,1)$ under the above-mentioned diffeomorphism, which we call the interior of $\Sigma$.

We also assume that each cross-section $\Sigma$ is contained in $U_{0}$, so that every $x \in \Sigma$ is such that $\omega(x) \subset \Lambda$. From now on we always assume that cross-sections are of this kind.

Given any two cross-sections $\Sigma$ and $\Sigma^{\prime}$ to the flow, a Poincaré map is a map defined by

$$
R: U \subset \Sigma \rightarrow \Sigma^{\prime}, \quad x \in U \mapsto X_{t(x)}(x) \in \Sigma^{\prime}
$$

(for a suitable hitting time $t$ which will be precised later). We note that, in general, $R$ needs not correspond to the first time the orbits of $\Sigma$ encounter $\Sigma^{\prime}$, nor it is defined everywhere in $\Sigma$. If $R$ is defined at $x \in \Sigma$, a time $t(x)>0$ so that $X_{t(x)}(x) \in \Sigma^{\prime}$ is called a Poincaré time of $x$.

The continuity of the flow implies that, if $R$ is defined at $x \in \Sigma$, then it is defined in an open neighborhood $U_{x}$ of $x$ in $\Sigma$ and it is a $C^{1}$ local diffeomorphism, see [36, Proposition 1.2, pp 94].

4.2.1. Hyperbolicity of Poincaré maps. Let $\Sigma$ be a cross-section to $X$ and $R: \Sigma \rightarrow \Sigma^{\prime}$ be a Poincaré map $R(y)=X_{t(y)}(y)$ to another cross-section $\Sigma^{\prime}$ (possibly $\Sigma=\Sigma^{\prime}$ ), defined as above.

The splitting $E^{s} \oplus E^{c u}$ over $U_{0}$ induces a continuous splitting $E_{\Sigma}^{s} \oplus E_{\Sigma}^{c u}$ of the tangent bundle $T \Sigma$ to $\Sigma$ (and analogously for $\Sigma^{\prime}$ ), defined by (recall (4.9) for the use of $E^{c s}$ )

$$
E_{\Sigma}^{s}(y)=E_{y}^{c s} \cap T_{y} \Sigma \quad \text { and } \quad E_{\Sigma}^{c u}(y)=E_{y}^{c u} \cap T_{y} \Sigma .
$$

The next result establishes that if the Poincaré time $t(x)$ is sufficiently large then (4.10) defines a hyperbolic splitting for the transformation $R$ on the cross-sections. Given a pair $\Sigma, \Sigma^{\prime}$ of crosssections in $\Xi$, we write $\Sigma\left(\Sigma^{\prime}\right)$ for the subset of points of $\Sigma$ whose Poincaré map is defined and hits $\Sigma^{\prime}$.

Proposition 7. 6, Proposition 6.15, pp 172] Let $R: \Sigma\left(\Sigma^{\prime}\right) \rightarrow \Sigma^{\prime}$ be a Poincaré map as before with Poincaré time $t(\cdot)$. Then $D R_{x}\left(E_{\Sigma}^{s}(x)\right)=E_{\Sigma}^{s}(R(x))$ at every $x \in \Sigma\left(\Sigma^{\prime}\right)$ and $D R_{x}\left(E_{\Sigma}^{c u}(x)\right)=$ $E_{\Sigma}^{c u}(R(x))$ at every $x \in \Lambda \cap \Sigma\left(\Sigma^{\prime}\right)$. 
Moreover, for every given $0<\lambda<1$ there exists $T_{1}=T_{1}\left(\Sigma, \Sigma^{\prime}, \lambda\right)>0$ such that if $t(\cdot)>T_{1}$ at every point, then

$$
\left\|D R \mid E_{\Sigma}^{s}(x)\right\|<\lambda \text { and }\left\|D R \mid E_{\Sigma}^{c u}(x)\right\|>1 / \lambda \quad \text { at every } x \in \Sigma\left(\Sigma^{\prime}\right) .
$$

Given a cross-section $\Sigma$, a positive number $\rho$, and a point $x \in \Sigma$, we define the unstable cone of width $\rho$ at $x$ by

$$
C_{\rho}^{u}(x)=\left\{v=v^{s}+v^{u}: v^{s} \in E_{\Sigma}^{s}(x), v^{u} \in E_{\Sigma}^{c u}(x) \text { and }\left\|v^{s}\right\| \leq \rho\left\|v^{u}\right\|\right\}
$$

(we omit the dependence on the cross-section in our notations). We note that $C_{\rho}^{u}(x)=C_{\rho}^{c u}(x) \cap$ $T_{x} \Sigma$.

Let $0<\rho<1$ be a small constant. In the following consequence of Proposition 7 (which is itself a consequence of partial hyperbolicity for the splitting $E^{s} \oplus E^{c u}$ ) we assume that the neighborhood $U_{0}$ has been chosen sufficiently small, depending on $\rho$ and on a bound on the angles between the flow and the cross-sections.

Corollary 4. 6, Corollary 6.17, pp 173] For $R: \Sigma \rightarrow \Sigma^{\prime}$ as in Proposition 7, with $t(\cdot)>T_{1}$, and any $x \in \Sigma\left(\Sigma^{\prime}\right)$, we have

$$
D R(x)\left(C_{\rho}^{u}(x)\right) \subset C_{\rho / 2}^{u}(R(x)) \quad \text { and } \quad\|D R(x)(v)\| \geq \frac{\lambda^{-1}}{2} \cdot\|v\| \quad \text { for all } \quad v \in C_{\rho}^{u}(x) .
$$

The proof of this corollary is based on the observation that, for small $\rho>0$, the vectors in $C_{\rho}^{u}(x)$ can be written as the direct sum of a vector in $E_{x}^{c u}$, which is expanded at a rate $\lambda^{-1}$, with a vector in $E_{x}^{c s}$, which is contracted at a rate $\lambda$. Hence, for small $\rho$, the center-unstable component dominates the stable component and the length of the vector is increased at a rate close to $\lambda^{-1}$.

In this way we can always achieve an arbitrarily large expansion rate along the directions of the unstable cone as long as we take $\lambda$ sufficiently close to zero and, consequently, a big enough threshold time $T_{1}$.

Let us introduce the following notion: a cu-curve in $\Sigma$ is a curve contained in a cross-section $\Sigma \in \Xi$ whose tangent direction $T_{z} \gamma$ is contained in a center-unstable cone $C_{\rho}^{u}(z) \subset T_{z} \Sigma$ for all $z \in \gamma$; see (4.11) below.

Remark 10. The cone $C_{a}^{c u}(x)$ is defined at every $x \in \Sigma$ and we can choose $\Sigma$ so that the diffeomorphism $h$ sends horizontal lines into cu-curves, i.e., curves whose tangent directions are contained in the cu-cone at every point.

4.2.2. Adapted cross-sections. The next step is to exhibit stable manifolds for Poincaré transformations $R: \Sigma \rightarrow \Sigma^{\prime}$. The natural candidates are the intersections $W^{s}(x, \Sigma)=W^{s}(x) \cap \Sigma$ we introduced previously. By construction, these leaves are contracted by the action of the flow and so they are contracted by the transformation $R$. Moreover, as already commented before, these intersections define a $C^{1+\alpha}$ stable foliation $\mathcal{F}_{\Sigma}^{s}$ of $\Sigma$ with a Hölder- $C^{1}$ holonomy. For our purposes it is also important that this foliation be invariant:

$$
R\left(W^{s}(x, \Sigma)\right) \subset W^{s}\left(R(x), \Sigma^{\prime}\right) \quad \text { for every } x \in \Lambda \cap \Sigma\left(\Sigma^{\prime}\right) .
$$

In order to have this we restrict our class of cross-sections so that the center-unstable boundary is disjoint from $\Lambda$. We recall (see Remark 9) that we are considering cross-sections $\Sigma$ that are diffeomorphic to the square $\mathbb{I} \times \mathbb{I}$, with the vertical lines $\{\eta\} \times \mathbb{I}$ being mapped to stable sets $W^{s}(y, \Sigma)$. The stable boundary $\partial^{s} \Sigma$ is the image of $\{0,1\} \times \mathbb{I}$. The center-unstable (or cu-)boundary $\partial^{c u} \Sigma$ is the image of $\mathbb{I} \times\{0,1\}$. The cross-section is $\delta$-adapted if

$$
d\left(\Lambda \cap \Sigma, \partial^{c u} \Sigma\right)>\delta,
$$

where $d$ is the intrinsic distance in $\Sigma$. We also recall that, from Remark 10 we choose the crosssections so that the $c u$-boundary is in fact formed by $c u$-curves.

We call vertical strip of $\Sigma$ the image $h(J \times \mathbb{I})$ for any compact subinterval $J$, where $h: \mathbb{I}^{2} \rightarrow \Sigma$ is the coordinate system on $\Sigma$ as in Remark 9. Notice that every vertical strip is an $\delta$-adapted cross-section. 


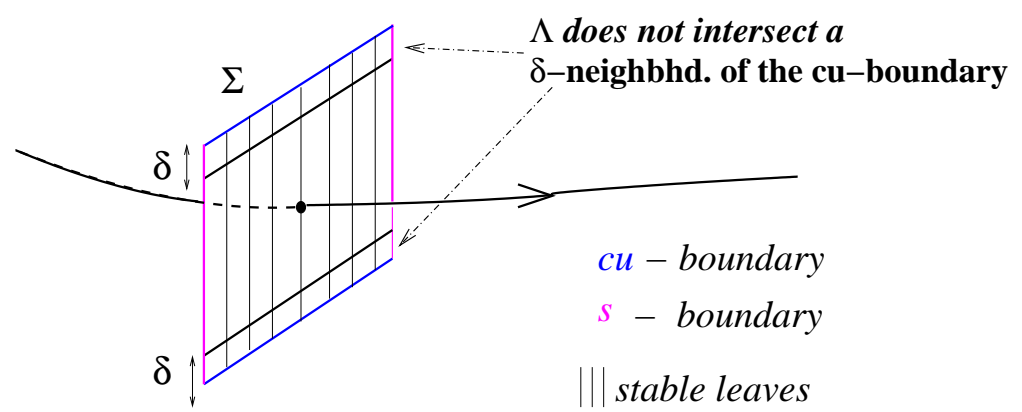

FigurE 1. An adapted cross-section for $\Lambda$.

Lemma 5. 6. Lemma 6.22, pp 177] Let $x \in \Lambda$ be a regular point, that is, such that $X(x) \neq 0$. Then there exists $\delta>0$ for which we can find a $\delta$-adapted cross-section $\Sigma$ at $x$.

Given cross-sections $\Sigma$ and $\Sigma^{\prime}$ we set $\Sigma\left(\Sigma^{\prime}\right)=\left\{x \in \Sigma: R(x) \in \Sigma^{\prime}\right\}$ the domain of the return map from $\Sigma$ to $\Sigma^{\prime}$. The next lemma establishes that if the cross-sections are adapted, then we have the invariance property (4.12).

Lemma 6. 6. Lemma 6.23, pp 178] Given $\delta>0$ and $\delta$-adapted cross-sections $\Sigma$ and $\Sigma^{\prime}$, there exists $T_{2}=T_{2}\left(\Sigma, \Sigma^{\prime}\right)>T_{1}>0$, where $T_{1}$ is as in Proposition 7 such that if $R: \Sigma\left(\Sigma^{\prime}\right) \rightarrow \Sigma^{\prime}$ defined by $R(z)=R_{t(z)}(z)$ is a Poincaré map with time $t(\cdot)>T_{2}$, then

(1) $R\left(W^{s}(x, \Sigma)\right) \subset W^{s}\left(R(x), \Sigma^{\prime}\right)$ for every $x \in \Sigma\left(\Sigma^{\prime}\right)$, and also

(2) $d(R(y), R(z)) \leq \frac{1}{2} d(y, z)$ for every $y, z \in W^{s}(x, \Sigma)$ and $x \in \Sigma\left(\Sigma^{\prime}\right)$.

This lemma provides a sufficient condition for having partial hyperbolicity for the Poincaré return map. Indeed, if $t>T_{2}>T_{1}$, then the stable leaves are sent strictly inside stable leaves and uniformly contracted by the ratio $1 / 2$; and the unstable cones on cross-sections are preserved.

4.2.3. Poincaré maps near Lorenz-like equilibria. Here we consider the Poincaré maps of the flow near the singularities.

We recall that, since the equilibria $\sigma=\sigma_{k}$ in our setting are all Lorenz-like, the unstable manifold $W^{u}(\sigma)$ is one-dimensional, and there is a one-dimensional strong-stable manifold $W^{s s}(\sigma)$ contained in the two-dimensional stable manifold $W^{s}(\sigma)$. By the smooth linearization results provided by Hartman 23] in the absence of resonances, orbits of the flow in a small neighborhood $U_{\sigma}$ of the given equilibrium $\sigma$ are solutions of the following linear system, modulo a smooth change of coordinates:

$$
(\dot{x}, \dot{y}, \dot{z})=\left(\lambda_{1} x, \lambda_{2} y, \lambda_{3} z\right) \quad \text { thus } \quad X_{t}\left(x_{0}, y_{0}, z_{0}\right)=\left(x_{0} e^{\lambda_{1} t}, y_{0} e^{\lambda_{2} t}, z_{0} e^{\lambda_{3} t}\right),
$$

with $\lambda_{2}<\lambda_{3}<0<-\lambda_{3}<\lambda_{1}$.

More precisely, let us consider and use the following smooth linearization result.

Theorem 7. Let $n \in \mathbb{Z}^{+}$be given. Then there exists an integer $N=N(n) \geq 2$ such that: if $\Gamma$ is a real non-singular $d \times d$ matrix with eigenvalues $\gamma_{1}, \ldots, \gamma_{d}$ satisfying

$$
\sum_{i=1}^{d} m_{i} \gamma_{i} \neq \gamma_{k} \quad \text { for all } \quad k=1, \ldots, d \quad \text { and } \quad 2 \leq \sum_{j=1}^{d} m_{j} \leq N
$$

and if $\dot{\xi}=\Gamma \xi+\Xi(\xi)$ and $\dot{\zeta}=\Gamma \zeta$, where $\xi, \zeta \in \mathbb{R}^{d}$ and $\Xi$ is of class $C^{N}$ for small $\|\xi\|$ with $\Xi(0)=0, \partial_{\xi} \Xi(0)=0$; then there exists a $C^{n}$ diffeomorphism $R$ from a neighborhood of $\xi=0$ to a neighborhood of $\zeta=0$ such that $R \xi_{t} R^{-1}=\zeta_{t}$ for all $t \in \mathbb{R}$ and initial conditions for which the flows $\zeta_{t}$ and $\xi_{t}$ are defined in the corresponding neighborhood of the origin.

Proof. See [23, Theorem 12.1, p. 257]. 
We recall that, in general, hyperbolic singularities are only linearizable by an at most Hölder homeomorphism according to the standard Hartman-Grobman Theorem [36, 42.

By Theorem 7 , hence it is enough for us to choose the eigenvalues $\left(\lambda_{1}, \lambda_{2}, \lambda_{3}\right) \in \mathbb{R}^{3}$ of $\sigma$ satisfying a finite set of non-resonance relations (4.14) for a certain $N=N(2)$ and for each singularity $\sigma_{k}$ in $\Lambda$. For this condition defines an open and dense set in $\mathbb{R}^{3}$ and so all small $C^{1}$ perturbations $Y$ of the vector field $X$ will have a singularity whose eigenvalues $\left(\lambda_{1}(Y), \lambda_{2}(Y), \lambda_{3}(Y)\right)$ are still in the $C^{2}$ linearizing region.

We note that in (4.13) $x_{1}$ corresponds to the strong-stable direction at $\sigma, x_{2}$ to the expanding direction and $x_{3}$ to the weak-stable direction.

Then for some $\delta>0$ we may choose cross-sections contained in $U_{\sigma}$

- $\Sigma_{\sigma}^{o, \pm}$ at points $y^{ \pm}$in different components of $W_{l o c}^{u}(\sigma) \backslash\{\sigma\}$

- $\Sigma_{\sigma}^{i, \pm}$ at points $x^{ \pm}$in different components of $W_{l o c}^{s}(\sigma) \backslash W_{l o c}^{s s}(\sigma)$

and Poincaré first hitting time maps $R^{ \pm}: \Sigma_{\sigma}^{i, \pm} \backslash \ell^{ \pm} \rightarrow \Sigma_{\sigma}^{o,-} \cup \Sigma_{\sigma}^{o,+}$, where $\ell^{ \pm}=\Sigma_{\sigma}^{i, \pm} \cap W_{l o c}^{s}(\sigma)$, satisfying (see Figure 2)

(1) every orbit in the attractor passing through a small neighborhood of the equilibrium $\sigma$ intersects some of the incoming cross-sections $\Sigma_{\sigma}^{i, \pm}$;

(2) $R^{ \pm}$maps each connected component of $\Sigma_{\sigma}^{i, \pm} \backslash \ell^{ \pm}$diffeomorphically inside a different outgoing cross-section $\Sigma_{\sigma}^{o, \pm}$, preserving the corresponding stable foliations.

Here we write $W_{l o c}^{*}(\sigma), *=s, s s, u$ for the local invariant stable, strong-stable and unstable manifolds of the hyperbolic saddle-type singularity $\sigma$ (see e.g. [36]), so that these invariant manifold extend up to the cross-sections $\Sigma^{i, \pm}$ and $\Sigma^{o, \pm}$.

We note that at each flow-box near a singularity there are four cross-sections: two "ingoing" $\Sigma_{\sigma}^{i, \pm}$ and two "outgoing" $\Sigma_{\sigma}^{o, \pm}$.

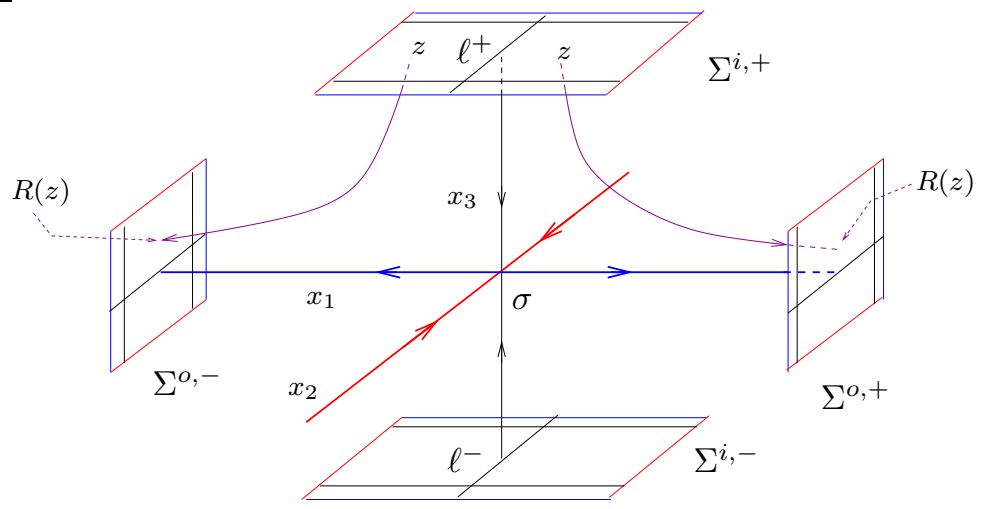

Figure 2. Cross-sections near a Lorenz-like equilibrium.

Using $C^{2}$ linearizing coordinates in a flow-box near a singularity, with the appropriate rescaling, we can assume without loss of generality that, for a small $\delta>0$, see Figure 2

$$
\begin{aligned}
& \Sigma^{i, \pm}=\left\{\left(x_{1}, x_{2}, \pm 1\right):\left|x_{1}\right| \leq \delta,\left|x_{2}\right| \leq \delta\right\} \quad \text { and } \\
& \Sigma^{o, \pm}=\left\{\left( \pm 1, x_{2}, x_{3}\right):\left|x_{2}\right| \leq \delta,\left|x_{3}\right| \leq \delta\right\} .
\end{aligned}
$$

Then from (4.13) we can determine the expression of the Poincaré maps between ingoing and outgoing cross-sections easily

$$
\Sigma^{i,+} \cap\left\{x_{1}>0\right\} \rightarrow \Sigma^{0,+}, \quad\left(x_{1}, x_{2}, 1\right) \mapsto\left(1, x_{2} \cdot x_{1}^{-\lambda_{2} / \lambda_{1}}, x_{1}^{-\lambda_{3} / \lambda_{1}}\right) .
$$

The cases corresponding to the other ingoing/outgoing pairs and signs of $x_{1}, x_{2}$ are similar.

This shows that the map obtained by identifying points with the same $x_{2}$ coordinate, i.e., points in the same stable leaf, is simply $x \mapsto f(x)=x^{\alpha}$ where $\alpha=-\lambda_{3} / \lambda_{1} \in(0,1)$. Analogously, the coordinate transverse to the stable leaves transforms according to the map $g(x, y)=y x^{\beta}$ where $\beta=-\lambda_{2} / \lambda_{1}>0$. 
Remark 11. Here $\partial_{x} g(x, y)=\beta y x^{\beta-1}$ is bounded if, and only if, $\beta \geq 1$ or, equivalently $-\lambda_{2}>\lambda_{1}$.

In these coordinates it is easy to see that for points $z=\left(x_{1}, x_{2}, \pm 1\right) \in \Sigma^{i, \pm}$ the time $\tau^{ \pm}$taken by the flow starting at $z$ to reach one of $\Sigma^{o, \pm}$ depends on $x_{1}$ only and is given by

$$
\tau^{ \pm}\left(x_{1}\right)=-\frac{\log \left|x_{1}\right|}{\lambda_{1}} \text { and consequently } \int_{-\delta}^{\delta}\left|\tau^{ \pm}\left(x_{1}\right)\right| d x_{1}<\infty
$$

This in particular shows that the return time on a ingoing cross-section near a singularity is constant on stable leaves.

4.3. Global Poincaré map. In this section we exibit a global Poincaré map for the flow near the singular-hyperbolic attractor $\Lambda$. The construction we perform here is slightly different from the one presented at [7]: we need injetiveness of the Poincaré return map to prove exact dimensionality of the physical measure. For this we need to cover the attractor by flow boxes through pairwise disjoint cross-sections and then consider a fixed iterate of the Poincaré first return map between these cross-sections. This is the main difference with respect to the usual construction presented elsewhere.

We observe first that by Lemma 5 we can take a $\delta$-adapted cross-section at each non-singular point $x \in \Lambda$. We know also that near each singularity $\sigma_{k}$ of $\Lambda$ there is a flow-box $U_{\sigma_{k}}$ containing $\sigma_{k}$ in its interior. Let $S(\Lambda)$ denote the finite set of equilibria contained in $\Lambda$, all of which are Lorenz-like.

Step 1: Choose a flow-box $U_{\sigma}$ near each singularity $\sigma \in S(\Lambda)$ as explained in Section 4.2 .3 with the extra conditions

(1) for any pair of distinct $\sigma_{1}, \sigma_{2} \in S(\Lambda)$ the flow-boxes

$$
\Sigma_{\sigma}^{i, \pm}(T):=\left\{X_{s}(x): x \in \operatorname{int}\left(\Sigma_{\sigma}^{*, \pm}\right),|s|<T_{1}\right\}, \quad \sigma=\sigma_{1}, \sigma_{2}, \quad *=i, o
$$

are pairwise disjoint, and

(2) the smallest time needed for the positive orbit of a point in $\Sigma^{i, \pm}$ to reach $\Sigma^{o, \pm}$ is bigger than $T_{1}$.

We note that since we may take $\Sigma_{\sigma}^{*, \pm}$ arbitrarily close to $\sigma$, these conditions can always be achieved. We denote by $\mathfrak{S}$ the family of all such cross-sections near the singularities of $\Lambda$.

Step 2: Consider the open set $V_{S}=\cup_{\sigma \in S(\Lambda)} \cup_{*=i, o} \Sigma_{\sigma}^{*, \pm}\left(T_{1}\right)$ and the compact subset $\Lambda_{1}:=$ $\Lambda \backslash V_{S}$ of $\Lambda$. For any $x \in \Lambda_{1}$ we know that $x$ is a regular point. Hence we have a $\delta$-adapted cross-section $\Sigma_{x}$ through $x$. We consider the $\varepsilon_{0}$-flow-box

$$
\Sigma_{x}\left(\varepsilon_{0}\right):=\left\{X_{s}(x): x \in \operatorname{int}\left(\Sigma_{x}\right),|s|<\varepsilon_{0}\right\}
$$

for a given fixed $\varepsilon_{0}>0$ small and $\varepsilon_{0}<T_{1}$. We note $x \in \Lambda_{1}$ ensures that $\Sigma_{x}\left(\varepsilon_{0}\right)$ does not contain any singularity and, in fact, does not intersect any of the cross-sections fixed at Step 1.

The collection $\mathcal{C}:=\left\{\Sigma_{x}\left(\varepsilon_{0}\right): x \in \Lambda_{1}\right\}$ is an open cover of the compact set $\Lambda_{1}$. We fix a finite subcover $\mathcal{C}_{0}=\left\{\Sigma_{x_{1}}\left(\varepsilon_{0}\right), \ldots, \Sigma_{x_{k}}\left(\varepsilon_{0}\right)\right\}$ in what follows and also consider the corresponding finite family of cross-sections $\Xi_{0}=\left\{\Sigma_{x_{1}}, \ldots, \Sigma_{x_{k}}\right\}$.

Step 3: Now we adjust the construction so that the Poincaré first return time between elements of $\Xi_{0}$ is bigger than some uniform positive constant.

For any given pair $\Sigma, \Sigma^{\prime} \in \Xi_{0}$, if we have $\operatorname{int}(\Sigma) \cap \operatorname{int}\left(\Sigma^{\prime}\right) \neq \emptyset$, then we may assume without loss of generality that the intersection is transversal. For otherwise, if $h: \mathbb{I}^{2} \rightarrow \Sigma$ is the coordinate system of $\Sigma$ given according to Remark 9 , we may find a $C^{1+\alpha}$ embedding $\tilde{h}: \mathbb{I}^{2} \rightarrow M$ close enough to $h$ so that $\widetilde{\Sigma}:=\tilde{h}\left(\mathbb{I}^{2}\right)$ is a $\delta$-adapted cross-section and there exists $\phi: \mathbb{I}^{2} \rightarrow\left(-\varepsilon_{0}, \varepsilon_{0}\right)$ such that $\tilde{h}(s, t)=X_{\phi(s, t)}(h(s, t))$ and both pairs $\operatorname{int}(\widetilde{\Sigma}), \operatorname{int}\left(\Sigma^{\prime}\right)$ and $\partial \widetilde{\Sigma}, \partial \Sigma^{\prime}$ are transversal. In particular, $\partial \widetilde{\Sigma}$ and $\partial \Sigma^{\prime}$ must be disjoint because the ambient space is three-dimensional. 
Hence we may assume that the (transversal) intersection $\Sigma \cap \Sigma^{\prime}$ is formed by finitely many smooth closed curves. We consider the sub-strip of $\Sigma$ given by

$$
\Sigma_{0}:=\cup\left\{W^{s}(y, \Sigma): y \in \Sigma \cap \Sigma^{\prime}\right\}
$$

and also the sub-strip of $\Sigma^{\prime}$ given by

$$
\Sigma_{1}:=\cup\left\{W^{s}\left(z, \Sigma^{\prime}\right): z \in \Sigma \cap \Sigma^{\prime}\right\}
$$

see Figure 3. According to the definition of $W^{s}(z, \Sigma), W^{s}\left(z, \Sigma^{\prime}\right)$ there are $\phi_{z}: W^{s}(z . \Sigma) \rightarrow$ $\left(-\varepsilon_{0}, \varepsilon_{0}\right)$ and $\tilde{\phi}_{z}: W^{s}\left(z, \Sigma^{\prime}\right) \rightarrow\left(-\varepsilon_{0}, \varepsilon_{0}\right)$ such that for each $z \in \Sigma \cap \Sigma^{\prime}$

$$
W_{0}^{s s}(z):=\left\{X_{\phi(x)}(x): x \in W^{s}(z, \Sigma)\right\} \cup\left\{X_{\tilde{\phi}(y)}(y): y \in W^{s}\left(z, \Sigma^{\prime}\right)\right\} \subset W_{\varepsilon}^{s s}(z)
$$

and $\phi(z)=0=\tilde{\phi}(z)$. Since, by Theorem [6] the stable manifolds in the neighborhood $U_{0}$ of $\Lambda$ depend $C^{2}$-smoothly on the base point

$$
\Sigma_{2}:=\left\{W_{0}^{s s}(z): z \in \Sigma \cap \Sigma^{\prime}\right\}
$$

is a $\delta$-adapted cross-section whose flow-box with time $2 \varepsilon_{0}$ covers the $\varepsilon_{0}$-flow-box of $\Sigma_{0}$ and $\Sigma_{1}$.

We replace $\Sigma$ and $\Sigma^{\prime}$ in $\Xi_{0}$ by the following strips: the closure of the connected components of $\Sigma \backslash \Sigma_{0}$; together with the closure of the connected components of $\Sigma^{\prime} \backslash \Sigma_{1}$; and the closure of $\Sigma_{2}$; see Figure 3. The number of such components is finite and, moreover, their flow boxes with time $2 \varepsilon_{0}$ cover at least the same portion of $\Lambda$ as the flow-boxes of $\Sigma$ and $\Sigma^{\prime}$.

This procedure ensures that, given any pair $\Sigma, \tilde{\Sigma}$ in $\Xi_{0}$, their interiors do not intersect, and the minimum Poincaré first return time between these sections is strictly positive. At this point we redefine $\mathcal{C}_{0}=\left\{\Sigma\left(2 \varepsilon_{0}\right): \Sigma \in \Xi_{0}\right\}$.

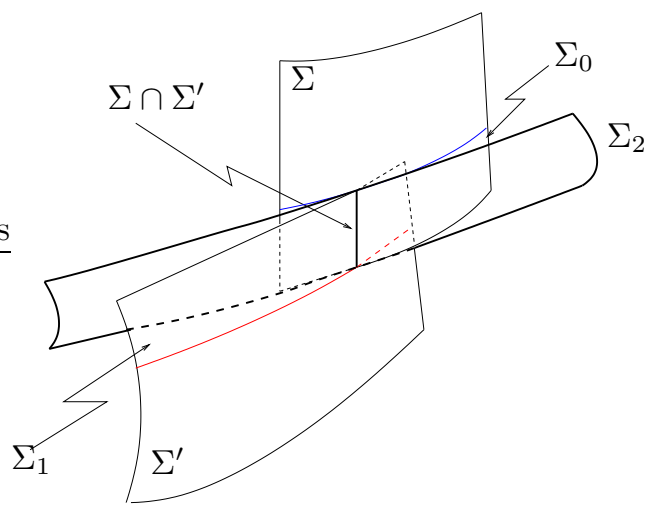

FiguRE 3. Cross-sections which intersect and their adaptation.

We define $\Xi:=\Xi_{0} \cup \mathfrak{S}$ the family of all cross-sections chosen in the above steps.

Remark 12. After this construction we note that

(1) we can ensure that each of the open sets $\Sigma\left(2 \varepsilon_{0}\right), \Sigma \in \Xi$ and $U_{\sigma}, \sigma \in S(\Lambda)$ is contained in the trapping region $U_{0}$, which we also assume is a neighborhood of $\Lambda$ where the extension of the strong-stable foliation is defined;

(2) given $\Sigma \in \Xi$ and $x \in \operatorname{int}(\Sigma)$, the Poincaré first return time for the positive orbit of $x$ to reach some cross-section in $\Xi$ is strictly positive; since the number of cross-sections is finite and each cross-section is compact, there exists $\varepsilon_{1}>0$ such that

$$
\inf \left\{t>0: X_{t}(x) \in \Xi\right\} \geq \varepsilon_{1} .
$$


(3) since $\Lambda$ is an attractor, the omega-limit set $\omega(z)$ of any $z \in \cup_{\Sigma \in \mathcal{C}} \Sigma\left(\varepsilon_{0}\right) \cup \cup_{\sigma \in S(\Lambda)} U_{\sigma}$ is contained in $\Lambda$. Let us assume that $z$ is a regular point. Thus, with the exception of the local stable manifolds of $\sigma$ in $U_{\sigma}$, a point $w \in \omega(z)$ has regular orbit under the flow which cross some cross-section in $\Xi$ in some future time. Therefore, the orbit of $z$, which accumulates in $\omega(z)$, must cross some cross-section of $\Xi$.

Definition 7. [Global Poincaré first return map] For any point $z$ in the interior of the crosssections in $\Xi$, we consider the first hit at a cross-section from $\Xi$. This gives the global Poincaré first return map

$$
R_{0}(z):=X_{\tau_{0}(z)}(z)
$$

and we say that

$$
\tau_{0}(z):=\inf \left\{t>0: X_{t}(z) \in \Xi\right\}
$$

is the Poincaré time of $z$. If the point $z$ never returns to one of the cross-sections, then the map $R_{0}$ is not defined at $z$.

Remark 13. This construction ensures that $R_{0}$ is injective, since it is a first return map between cross-sections of a flow.

In this way we cannot yet ensure that the Poincaré time is big enough to guarantee hyperbolicity of the return map. To obtain such big enough Poincaré time we consider an iterate of $R_{0}$, as follows. Using Proposition 7 and Lemma [ 6 for the collection $\Xi$ of $\delta$-adapted cross-sections, we consider the following threshold time

$$
T:=\max \left\{T_{1}, T_{2}\left(\Sigma, \Sigma^{\prime}\right): \Sigma, \Sigma^{\prime} \in \Xi, \Sigma \neq \Sigma^{\prime}\right\} .
$$

Hence, if we choose a big enough iterate $R_{0}^{N}$ of the Poincaré first return map so that the return time for $R=R_{0}^{N}$ is bigger than $T$, then the tangent map to $R: \Sigma\left(\Sigma^{\prime}\right) \rightarrow \Sigma^{\prime}$ is hyperbolic between any pair of cross-sections.

Definition 8. [Global Poincaré map] We choose $N \in \mathbb{Z}^{+}$such that $N \varepsilon_{1}>T$ and set $R:=R_{0}^{N}$.

We note that $R$ in the definition above is guaranteed to have hyperbolic derivative at every point where it is defined, since we can write

$$
R(z)=X_{\tau(z)}(z) \quad \text { with } \quad \tau(z)=\sum_{i=0}^{N-1} \tau_{0}\left(R_{o}^{i}(z)\right)>T
$$

if $R(z)$ is defined, $z \in \Xi$. The function $\tau$ is the global Poincaré time and (4.19) shows that both Proposition 7 and Lemma 6 simultaneously hold.

In addition, by Lemma 6, if $R$ is defined for $x \in \Sigma$ on some $\Sigma \in \Xi$, then $R$ is defined for every point in $W^{s}(x, \Sigma)$. Hence the domain of $R \mid \Sigma$ consists of strips of $\Sigma$. The smoothness of $(t, x) \mapsto X_{t}(x)$ ensures that the strips

$$
\Sigma\left(\Sigma^{\prime}\right)=\left\{x \in \Sigma: R(x) \in \Sigma^{\prime}\right\}
$$

have non-empty interior in $\Sigma$ for every $\Sigma, \Sigma^{\prime} \in \Xi$.

Remark 14. Since $R$ is a fixed iterate of the injective map $R_{0}$, we see that $R$ is also injective. Moreover, by item (3) of Remark [12, the family of all $\Sigma\left(\Sigma^{\prime}\right)$ for $\Sigma^{\prime} \in \Xi$ covers $\Sigma$ except the points where $R$ is not defined.

4.3.1. Finite number of strips in the domain of the global Poincaré return map. The next result shows that, fixing a cross-section $\Sigma \in \Xi$, the points where $R$ is not defined are contained in finitely many stable leaves. Thus, after Remark 14, the family of all possible strips, defined as in (4.20) by the set of points $\Sigma\left(\Sigma^{\prime}\right)$ which move from $\Sigma$ to some strip $\Sigma^{\prime} \in \Xi$, covers $\Sigma$ except for finitely many stable leaves $W^{s}\left(x_{i}, \Sigma\right), i=1, \ldots, m=m(\Sigma)$. Thus the number of strips in each cross-section is finite.

We note that $R$ is locally smooth for all points $x \in \operatorname{int}(\Sigma)$ such that $R(x) \in \operatorname{int}(\Xi)$ by the Tubular Flow Theorem, [36, Theorem 1.1, pp 40], and the smoothness of the flow, where int $(\Xi)$ 
is the union of the interiors of each cross-section of $\Xi$. Let $\partial^{s} \Xi$ denote the union of all the leaves forming the stable boundary of every cross-section in $\Xi$.

Lemma 7. [6. Lemma 6.29, pp 182] The subset $\mathcal{D}$ of points for which $R$ is not defined in $\Xi \backslash \partial^{s} \Xi$ is contained in the set of points $x \in \Xi \backslash \partial^{s} \Xi$ such that:

(a) either $R(x)$ is defined and belongs to $\partial^{s} \Xi$;

(b) or there is some time $0<t \leq t_{2}, t_{2}$ given at Lemma [6, such that $X_{t}(x) \in W_{\varepsilon}^{s}(\sigma) \cap \Sigma_{j}$ for some singularity $\sigma$ of $\Lambda$ and $\Sigma_{j} \in \Xi$.

Moreover this set is contained in a finite number of stable leaves of the cross-sections $\Sigma \in \Xi$.

The proof of this lemma depends on the fact that the Poincaré time is finite for points where $R(x)$ is defined, so the other points must be inside the attractor but never cross other cross-sections of $\Xi$, so they must be converging to an equilibrium point of $X$ in $\Lambda$; or they will hit the stable boundary of some cross-section of $\Xi$.

Let $\Gamma$ be the finite set of stable leaves of $\Xi$ provided by Lemma 7 together with $\partial^{s} \Xi$. Then the complement $\Xi \backslash \Gamma$ of this set is formed by finitely many open strips $\Sigma \in \Xi$, where $R$ is smooth, i.e., of class $C^{2}$. Each of these strips is then a connected component of the sets $\Sigma\left(\Sigma^{\prime}\right)$ for $\Sigma, \Sigma^{\prime} \in \Xi$, where $R$ is a $C^{2}$ diffeomorphism.

4.3.2. Integrability of the global Poincaré return time $\tau$. We may now obtain a crucial property for the construction of the physical measure for singular hyperbolic attractors and to study its properties in what follows.

Lemma 8. The global Poincaré time $\tau$ is integrable with respect to the Lebesgue area measure in $\Xi$, induced by the Riemannian Lebesgue volume form on the manifold.

Proof. Indeed, given $z \in \Xi$, the point $R(z)=X_{\tau(z)}(z)$ is also given by $R_{0}^{N}(z)=X_{S_{N} \tau_{0}(z)}(z)$ where $\tau(z)=S_{N} \tau_{0}(z)=\sum_{k=0}^{N-1} \tau_{0}\left(R_{0}^{k}(z)\right)$. Hence $\tau$ is bounded by the sum of at most $N$ exit-time functions of flow-boxes of $S(\Lambda)$ (all of them integrable with respect to Lebesgue measure) plus the sum of at most $N$ bounded Poincaré first return time functions between cross-sections in $\mathcal{C}_{0}$, away from singularities. Thus the Poincaré time of $R$ on $\Xi$ is Lebesgue integrable.

4.4. The two-dimensional map $F$. ¿From now on, $\Xi$ is the collection of all strips $\Sigma\left(\Sigma^{\prime}\right)$ where the Poincaré return map is smooth. We still denote the strips by the letter $\Sigma$ in what follows. We choose a $C^{2}$ cu-curve $\gamma_{\Sigma}$ transverse to $\mathcal{F}_{\Sigma}^{s}$ in each $\Sigma \in \Xi$. Then the projection $p_{\Sigma}$ along leaves of $\mathcal{F}_{\Sigma}^{s}$ onto $\gamma_{\Sigma}$ is a $C^{1+\alpha}$ map, since the stable leaves $W^{s}(x, \Sigma)$ are defined through every point of $\Sigma \in \Xi$ and holonomies depend $C^{1+\alpha}$ smoothly on the base point.

Given a set $A, \operatorname{Cl}(A)$ means the closure of $A$. We define

$$
I=\bigcup_{\Sigma, \Sigma^{\prime} \in \Xi} \mathrm{Cl}\left(\Sigma\left(\Sigma^{\prime}\right)\right) \cap \gamma_{\Sigma} \quad \text { and } \quad S=\bigcup_{\Sigma, \Sigma^{\prime} \in \Xi} \mathrm{Cl}\left(\Sigma\left(\Sigma^{\prime}\right)\right) .
$$

As the number of strips is finite, by the properties of $\Sigma\left(\Sigma^{\prime}\right)$ obtained earlier, the set $I$ is $C^{2}$ diffeomorphic to a closed interval $\mathbb{I}=[0,1]$ with finitely many points $\mathcal{C}=\left\{c_{1}, \ldots, c_{n}\right\}$ removed, and $p_{\Sigma} \mid p_{\Sigma}^{-1}(I)$ becomes a $C^{1}$ submersion. The set $S$ is $C^{1+\alpha}$-diffeomorphic to a non-degenerate closed rectangle $Q \subset \mathbb{R}^{2}, Q=[0,1] \times[0,1]$ with finitely many vertical lines $\mathcal{C} \times \mathbb{I}=\left\{c_{1}, \ldots, c_{n}\right\} \times \mathbb{I}$ removed. We denote by $H$ the $C^{1+\alpha}$-diffeomorphism $H: S \rightarrow Q$ which sends stable leaves to vertical lines and consider the composition map

$$
F=H \circ R \circ H^{-1}: Q \rightarrow Q .
$$

According to Lemma 6 Proposition 7 and Corollary 4 the Poincaré map $R: \Xi \backslash \Gamma \rightarrow \Xi$ takes stable leaves of $\mathcal{F}_{\Sigma}^{s}$ inside stable leaves of the same foliation and is $C^{1}$ piecewise hyperbolic. In addition, by Corollary 4 a $c u$-curve $\gamma \subset \Sigma$ is taken by $R$ into a $c u$-curve $R(\gamma)$ in the image cross-section.

We can define unstable cones on $Q$ using the smoothness of $H$ as $\mathbb{C}_{\rho}^{u}(H(x)):=D H(x) \cdot C_{\rho}^{u}(x)$. This ensures, in particular, that $c u$-curves are taken by $F=H \circ R \circ H^{-1}$ into $c u$-curves. Hence, the map $F=H \circ R \circ H^{-1}: Q \rightarrow Q$ can be written as

$$
F(x, y)=(T(x), G(x, y)),
$$


where

$$
T: \mathbb{I} \backslash \mathcal{C} \rightarrow \mathbb{I}, \quad(\mathbb{I} \backslash \mathcal{C}) \ni z \mapsto H\left(p_{\Sigma^{\prime}}\left(R\left(W^{s}\left(H^{-1}(z), \Sigma\right) \cap \Sigma\left(\Sigma^{\prime}\right)\right)\right)\right)
$$

Moreover, by construction, we have that the following hold:

(a) $T: \mathbb{I} \backslash \mathcal{C} \rightarrow \mathbb{I}$ is not defined at a finite number of points $c_{1}, \cdots, c_{n}$, and it is $C^{1}$ at $\mathbb{I} \backslash \mathcal{C}=\cup_{0 \leq j \leq n} I_{j}$. The points $c_{1}, \cdots, c_{n}$ correspond either to the projection of a line $\ell=\Sigma_{i} \cap W_{\varepsilon}^{s}(\bar{\sigma})$ of points which fall in the stable manifold of an equilibrium $\sigma$, or to the projection of the boundary of a strip $\Sigma \in \Xi$.

(b) $G: Q \rightarrow \mathbb{I}$ is not defined at a finite number of vertical lines $\ell_{c_{i}}=\left\{c_{i}\right\} \times \mathbb{I}$ in $Q$, corresponding to $p_{\Sigma}^{-1}\left(c_{i}\right)$, where $c_{i}$ are as in (a) and $G$ is $C^{1}$ restricted to $Q \backslash\left(\cup_{1 \leq i \leq n} \ell_{c_{i}}\right)$.

(c) the choice of $R$ as an iterate of the first return map $R_{0}$ between the finite family $\Xi$ of cross-sections ensures that $F$ is injective in the following sense: if $i \neq j$ then $F\left(\left(c_{i}, c_{i+1}\right) \times\right.$ $[0,1]) \cap F\left(\left(c_{j}, c_{j+1}\right) \times[0,1]\right)=\emptyset$.

Finally, with the convention $c_{0}=0$ and $c_{n}=1$, the restriction of $F$ to each strip $\Sigma_{j}=\left(c_{j}, c_{j+1}\right) \times$ $[0,1] \subset Q, 0 \leq j \leq n-1$, is given by

$$
F_{\Sigma_{j}}(x, y)=\left(T_{\Sigma_{j}}(x), G_{\Sigma_{j}}(x, y)\right)
$$

We note that Lemma 6(b) together with the fact that $H$ is a $C^{1+\alpha}$-diffeomorphism imply

Lemma 9. The map $F: Q \rightarrow Q$ preserves the vertical foliation $\mathcal{F}^{s}$ of $Q$, and $F \mid \gamma$ is $\lambda$-Lipschitz with $\lambda<1$ on each leaf $\gamma \in \mathcal{F}^{s}$.

Remark 15. By taking the cross-sections $\Sigma$ small enough we can ensure that the unstable cone has very small variation along the strip. We can then ensure that all curves which are at a constant distance from $\gamma_{\Sigma}$ along $\mathcal{F}_{\Sigma}^{s}$ are also cu-curves. Since $\mathcal{F}_{\Sigma}^{s}$ is sent to vertical lines in $Q$ through $H$, this in turn ensures that all horizontal lines in $Q$ which do not intersect the vertical lines $\ell_{c_{i}}$ are cu-curves, because these horizontal lines correspond through $H$ to curves which are essentially at a constant distance from $\gamma_{\Sigma}$ along the stable leaves.

4.4.1. Additional properties of the one-dimensional map $T$. As already mentioned, since the flow $\left(X_{t}\right)_{t \in \mathbb{R}}$ is $C^{2}$, the leaves $W^{s}(x, \Sigma), x \in \Sigma$, define a $C^{1}$ foliation $\mathcal{F}_{\Sigma}^{s}$ of each $\Sigma \in \Xi$ with a Hölder- $C^{1}$ holonomy (since the leaves are one-dimensional).

These properties taken together with the expansion provided by Corollary 4 imply (see the proof in [6, Sec. 7.3.2, pp 222]).

Lemma 10. The one-dimensional map $T$ obtained above is in fact a $C^{1+\alpha}$ piecewise expanding map such that $1 /|D T|$ is $\alpha$-Hölder, for some $0<\alpha<1$, restricted to each $I_{j}$.

The uniform expansion is a consequence of the existence of a uniform bound for the angles between $\mathcal{F}_{\Sigma}^{s}$ and the curves $\gamma_{\Sigma}$, once we have fixed the set $\Xi$ of cross-sections, and our ability to obtain an arbitrarily large expansion rate along the unstable cones if we choose the threshold $T_{1}>0$ large enough.

As seen in Section 3 (see Theorem 4 and consequences), if $T$ is piecewise expanding and $h=$ $1 /|D T|$ has finite universal $p$-bounded variation then there is an absolutely continuous invariant measure with $p$-bounded variation density. It is easy to see that if $1 /|D T|$ is piecewise $\alpha$-Hölder for some $\alpha \in(0,1)$, then it is of universal $p$-bounded variation. Moreover, by taking an iterate $T^{k}$ of $T$ if necessary, we can assume that each ergodic absolutely continuous invariant probability measure for $T$ is decomposed into a finite family of of probability measures invariant for $T^{k}$ and having exponential speed of convergence to equilibrium.

Thus, Lemma 10 together with the results from Section 3 imply the following result.

Lemma 11. The one-dimensional map T obtained above has finitely many ergodic physical measures $\mu_{T}^{1}, \ldots, \mu_{T}^{l}$, whose density is a function of $p$-bounded variation, and whose ergodic basins cover Lebesgue almost all points of $\mathbb{I}$. 
We recall that, given a $\varphi$-invariant Borel probability measure $\mu$ with respect to a map $\varphi: X \circlearrowleft$ on a metric space $X$, we denote by

$$
B(\mu)=\left\{x \in X: \lim _{n \rightarrow+\infty} \frac{1}{n} \sum_{j=0}^{n-1} \psi\left(\varphi^{j}(x)\right)=\int \phi d \mu, \quad \forall \psi \in C^{0}(X, \mathbb{R})\right\}
$$

the ergodic basin of $\mu$ and say that $\mu$ is physical if the volume of $B(\mu)$ (or some other natural measure) is positive.

According to standard constructions described in [7] and [6, Section 7.3, pp. 225-235], each absolutely continuous ergodic probability measure $\mu_{T}^{i}$ for $T$ can be lifted to a unique physical ergodic probability measure $\mu_{F}^{i}$ for the map $F$, in such a way that $\pi_{*} \mu_{F}^{i}=\mu_{T}^{i}$, where $\pi: Q \rightarrow \mathbb{I}$ is the projection on the first coordinate and we have $\pi \circ F=T \circ \pi$. Hence the ergodic basin $B\left(\mu_{F}^{i}\right)$ of $\mu_{F}^{i}$ is given by $\pi^{-1}\left(\operatorname{supp} \mu_{T}^{i}\right)$ and is a finite collection of strips with non-empty interior and the interior of the supports of distinct $\mu_{F}^{j}$ and $\mu_{F}^{i}$ are disjoint.

Moreover, each probability measure $\mu_{F}^{i}$ can be lifted to a physical ergodic probability measure $\nu_{\Lambda}^{i}$ for the flow of $X$ supported in the attractor $\Lambda$; more on this in Subsection 4.5. Since a singularhyperbolic attractor is transitive, that is, it has a dense orbit, it follows that there can be only one such physical measure for the flow in the basin of attraction of $\Lambda$; see [6. Section 7.3.8, pp. 234-235].

Lemma 12. For each absolutely continuous ergodic probability measure $\mu_{T}^{i}$ for $T$ there exists a unique physical ergodic probability measure $\mu_{F}^{i}$ for the map $F$ such that $\pi_{*} \mu_{F}^{i}=\mu_{T}^{i}$.

For each physical measure $\mu_{F}^{i}$ for $F$ there exists a unique ergodic physical measure $\nu_{X}$ for $X$.

We note that since $F$ is given by a power of the first return map $R_{0}$, the uniqueness statement above does not imply uniqueness of the absolutely continuous invariant probability measure $\mu_{T}$ for $T$.

4.4.2. Positive entropy for the two-dimensional map. We will also need the fact that the entropy $h_{\mu_{F}^{i}}(F)$ of the map $F$ with respect to each physical measure $\mu_{F}^{i}$ is positive. Since we know that $\pi \circ F=T \circ \pi$ and $h_{\mu_{T}^{i}}(T)=\int \log |D T| d \mu_{T}>0$, where $\mu_{T}^{i}$ is one ergodic absolutely continuous $T$ invariant probability measure, we see that $h_{\mu_{F}^{i}}(F)>0$, for each $F$-invariant ergodic and physical probability measure $\mu_{F}^{i}$.

4.4.3. Additional properties of the map $G$. Since $F(x, y)=(T(x), G(x, y))$, Lemma 9 means that there is $0<\lambda<1$ such that for all fixed $x$,

$$
\operatorname{dist}\left(G\left(x, y_{1}\right), G\left(x, y_{2}\right)\right) \leq \lambda \cdot\left|y_{1}-y_{2}\right|, \forall y_{1}, y_{2} \in \mathbb{I} .
$$

The form of the singularities ensures the following result.

Lemma 13. If for all singularities $\sigma$ we have that the eigenvalues $\lambda_{2}(\sigma)<\lambda_{3}(\sigma)<0<\lambda_{1}(\sigma)$ satisfy the non-resonance conditions expressed in Theorem [7, then the map $G: Q \rightarrow \mathbb{I}$ satisfies $\operatorname{var}^{\square}(G)<\infty$.

Proof. From the choices made in Section 4.2 .3 we see that the expression of the Poincaré maps between ingoing and outgoing cross-sections implies the following.

For a maximal interval $\left(c_{i}, c_{i+1}\right)$, where the first coordinate map $T$ is monotonous, corresponding to an ingoing cross-section near a equilibrium point of the flow $\sigma_{i}$ with the eigenvalue ratios $\alpha_{i}>0$ and $\beta_{i} \in(0,1)$, we have that $F \mid\left(c_{i}, c_{i+1}\right) \times \mathbb{I}$ is given by

$$
(x, y) \mapsto\left(\left(x-c_{i}\right)^{\beta}, y\left(x-c_{i}\right)^{\alpha_{i}}\right) \quad \text { or } \quad(x, y) \mapsto\left(\left|x-c_{i+1}\right|^{\beta}, y\left|x-c_{i+1}\right|^{\alpha_{i}}\right)
$$

For the remaining cases, $F \mid\left(c_{i}, c_{i+1}\right) \times \mathbb{I}$ is just a Poincaré map between tubular neighborhoods of regular points for the flow, whose derivatives are bounded: $T^{\prime}, \partial_{x} G$ and $\partial_{y} G$ are bounded functions. Since there are finitely many such tubular neighborhoods, we let $K>0$ be an upper bound for these derivatives.

Let us now consider the estimation of the total variation. Let $0=x_{1}<x_{2}<\ldots,<x_{n}=1$ be a partition of $\mathbb{I}$ and $y_{1}, y_{2}, \ldots, y_{n}$ arbitrary points in $\mathbb{I}$. For a sequence $x_{k}<x_{k+1}<\cdots<x_{l}$ in $\left(c_{i}, c_{i+1}\right)$ we consider two cases. 
Case A: $\left(c_{i}, c_{i+1}\right) \times \mathbb{I}$ is the domain corresponding to an ingoing cross-section near an equilibrium point of the flow. We can write, since $\left(x_{j}, y_{j}\right) \in Q$

$$
\begin{aligned}
\sum_{k \leq j \leq l}\left|G\left(x_{j}, y_{j}\right)-G\left(x_{j+1}, y_{j}\right)\right| & =\sum_{k \leq j \leq l} y_{j}\left(\left(x_{j+1}-c_{i}\right)^{\alpha_{i}}-\left(x_{j}-c_{i}\right)^{\alpha_{i}}\right) \\
& \leq \sum_{k \leq j \leq l}\left(\left(x_{j+1}-c_{i}\right)^{\alpha_{i}}-\left(x_{j}-c_{i}\right)^{\alpha_{i}}\right) \\
& =\left(x_{l}-c_{i}\right)^{\alpha_{i}}-\left(x_{k}-c_{i}\right)^{\alpha_{i}} \leq 1
\end{aligned}
$$

where we have assumed that $G(x, y)=y\left(x-c_{i}\right)^{\alpha_{i}}$. The other case, with $c_{i+1}$ in the place of $c_{i}$, is similar.

Case B: $\left(c_{i}, c_{i+1}\right) \times \mathbb{I}$ is the domain corresponding to a tubular neighborhood away from a singular flow-box. We can now write by the Mean Value Theorem

$$
\sum_{k \leq j \leq l}\left|G\left(x_{j}, y_{j}\right)-G\left(x_{j+1}, y_{j}\right)\right|=\sum_{k \leq j \leq l}\left|\partial_{x} G\left(x_{j}^{*}, y_{j}\right)\right|\left(x_{j+1}-x_{j}\right) \leq K\left(x_{l}-x_{k}\right) \leq K
$$

for some $x_{j}^{*} \in\left(x_{j}, x_{j+1}\right)$.

Finally, the case $x_{k}<c_{i}<x_{k+1}$ can also be bounded

$$
\left|G\left(x_{k}, y_{k}\right)-G\left(x_{k+1}, y_{k}\right)\right| \leq 2 \sup _{x \in \mathbb{I}}\left|g\left(x, y_{k}\right)\right| \leq 2 .
$$

We note that the bounds we have obtained do not depend on the choice of the $y_{j}$. Since there are finitely many such tubular neighborhoods and flow-boxes to consider, we see that

$$
\sup _{n \in \mathbb{Z}^{+}} \sup _{\substack{y_{1}, \ldots, y_{n} \in \mathbb{I} \\ 0=x_{1}<\cdots<x_{n}=1}} \sum_{j=1}^{n}\left|G\left(x_{j}, y_{j}\right)-G\left(x_{j+1}, y_{j}\right)\right|
$$

is bounded above by a constant times the number of smooth branches of the first coordinate function $f$.

Now we state a straightforward consequence of Remark 11

Lemma 14. If for all singularities $\sigma \in \Lambda$ we have the eigenvalue relation $-\lambda_{2}(\sigma)>\lambda_{1}(\sigma)$, then the second coordinate map $G$ of $F$ has a bounded partial derivative with respect to the first coordinate, i.e., there exists $C>0$ such that $\left|\partial_{x} G(x, y)\right|<C$ for all $(x, y) \in\left(\mathbb{I} \backslash\left\{c_{1}, \ldots, c_{n}\right\}\right) \times \mathbb{I}$.

Proof. We just have to observe that in the domains corresponding to ingoing cross-sections near singularities, the expression of the map $g$ is given by (4.22). Thus we can apply Remark 11] to conclude that $\partial_{x} g$ is bounded if, and only if, the stated eigenvalue relation holds for the eigenvalues of $D X$ at the equilibrium point. For the other domains, since $F$ is just a Poincaré map between tubular neighborhoods of regular points for the flow, its partial derivatives are bounded.

4.5. Integrability of $\tau, \log \left|T^{\prime}\right|$ and $\log \left|\partial_{y} G\right|$ with respect to the physical measures. Now we show that the global Poincaré time $\tau$ is integrable with respect to the $F$-invariant physical measures $\mu_{F}^{i}$ on $Q$, which lifts to the physical measure $\nu$ for the flow on the singular-hyperbolic attractor and itself is a lift of the $T$-invariant absolutely continuous probability measure $\mu_{T}$ on $\mathbb{I}$.

Proposition 8. The global Poincaré time $\tau$ is integrable with respect to each $F$-invariant physical probability measure $\mu_{F}^{i}$.

Proof. We recall the main steps of the construction of $\mu_{F}$ from $\mu_{T}$.

We denote by $\mathcal{F}=\{\{x\} \times \mathbb{I}\}_{x \in \mathbb{I}}$ the vertical foliation on the square $Q$ and by $\Gamma=\left\{\left\{c_{i}\right\} \times \mathbb{I}\right\}_{i=1, \ldots, n}$ the leaves corresponding to discontinuities of the map $T$. We have already shown that $\mathcal{F}$ is

- invariant: the image of any $\xi \in \mathcal{F}$ distinct from $\Gamma$ is contained in some element $\eta$ of $\mathcal{F}$;

- contracting: the diameter of $F^{n}(\xi)$ goes to zero when $n \rightarrow \infty$, uniformly over all the $\xi \in \mathcal{F}$ for which $F^{n}(\xi)$ is defined. 
Let $\mu_{T}^{i}$ be an absolutely continuous probability measure on $\mathbb{I}=Q / \mathcal{F}$ invariant under the transformation $T$ obtained in Subsection 4.4.1. For each bounded function $\psi: Q \rightarrow \mathbb{R}$, let $\psi_{-}: \mathcal{F} \rightarrow \mathbb{R}$ and $\psi_{+}: \mathcal{F} \rightarrow \mathbb{R}$ be defined by

$$
\psi_{-}(\xi)=\inf _{x \in \xi} \psi(x) \quad \text { and } \quad \psi_{+}(\xi)=\sup _{x \in \xi} \psi(x) .
$$

Lemma 15. [6, Lemma 7.21] Given any continuous function $\psi: Q \rightarrow \mathbb{R}$, both limits

$$
\lim _{n} \int\left(\psi \circ F^{n}\right)_{-} d \mu_{T}^{i} \quad \text { and } \quad \lim _{n} \int\left(\psi \circ F^{n}\right)_{+} d \mu_{T}^{i}
$$

exist, and they coincide.

¿From this it is straighforward to prove the following.

Corollary 5. [6, Corollaries 7.22, 7.25 \& Subsection 7.3.5] There exists a unique probability measure $\mu_{F}^{i}$ on $Q$ such that

$$
\int \psi d \mu_{F}^{i}=\lim \int\left(\psi \circ F^{n}\right)_{-} d \mu_{T}^{i}=\lim \int\left(\psi \circ F^{n}\right)_{+} d \mu_{T}^{i}
$$

for every continuous function $\psi: \Xi \rightarrow \mathbb{R}$. Besides, $\mu_{F}^{i}$ is invariant under $F$ and is $F$-ergodic. In addition, the basin $B\left(\mu_{F}^{i}\right)$ of $\mu_{F}$ equals $Q$ except for a zero Lebesgue (area) measure subset.

Now, for the integrability of $\tau$ with respect to $\mu_{F}^{i}$, we use the construction in Lemma 15. We observe first that for each $(x, y) \in Q \backslash \Gamma$ we have a constant $c>0$ such that

$$
\left(\tau \circ F^{n}(x, y)\right)_{+}-\left(\tau \circ\left(F^{n}(x, y)\right)_{-}<c \cdot \operatorname{diam}\left(F^{n}(\{x\} \times \mathbb{I})\right)\right.
$$

since, as explained in Subsection 4.3, the Poincaré time on points of a ingoing cross-section near a equilibrium point is constant on the stable leaves; and on other cross-sections is just a uniformly bounded smooth function of $y$. Moreover, because the density $d \mu_{T}^{i} / d m$ is bounded from above (since it is a function of generalized bounded variation on the interval; see Section 3), by a constant $L>0$ say, we have

$$
\int \tau_{+} d \mu_{T}^{i} \leq L \int \tau_{+} d m<\infty
$$

since, as explained in (4.16), the integral with respect to Lebesgue (length) measure over an ingoing cross-section is finite, and the return time function is globally bounded otherwise.

Hence we may define $\tau_{\mathbb{I}}(x):=\tau(x, y)_{+}$and write

$$
\tau_{\mathbb{I}}\left(T^{n}(x)\right)=\left(\tau\left(F^{n}(x, y)\right)\right)_{+} \geq \tau\left(F^{n}(x, y)\right)-c \cdot \operatorname{diam}\left(F^{n}(\{x\} \times \mathbb{I})\right) \geq \tau\left(F^{n}(x, y)\right)-c \lambda^{n}
$$

for all $y \in \mathbb{I}, n \geq 1$ and $\mu_{T}$-almost every $x \in \mathbb{I}$. Thus we obtain

$$
\int\left(\tau \circ F^{n}\right)_{-} d \mu_{T}^{i} \leq \int\left(\tau_{\mathbb{I}} \circ T^{n}\right) d \mu_{T}^{i}+c \lambda^{n}=\int \tau_{\mathbb{I}} d \mu_{T}^{i}+c \lambda^{n}
$$

and from Lemma 15] we see that $\int \tau d \mu_{F}^{i} \leq \int \tau_{\mathbb{I}} d \mu_{T}^{i}<\infty$. This completes the proof of the proposition.

Some other integrability or regularity properties will be needed in the sequel. We obtain them here.

Proposition 9. We have the following properties:

(1) $0<\int \log \left|T^{\prime}\right| d \mu_{F}<\infty$;

(2) $\int-\log \left|\partial_{y} G(x, y)\right| d \mu_{F}<\infty$;

(3) the maps $y \mapsto \partial_{y} G(x, y)$ are uniformly equicontinuous for $x \in \mathbb{I} \backslash\left\{c_{1}, \ldots, c_{n}\right\}$, i.e., outside the singularities of the map $T$. 
Proof. For the integrability, we repeat the arguments in the proof of Proposition 8

Indeed, at a maximal interval $\left(c_{i}, c_{i+1}\right)$, where the first coordinate map $T$ is monotonous, corresponding to an ingoing cross-section near a equilibrium point $\sigma_{i}$ with the eigenvalue ratios $\alpha_{i}>0$ and $\beta_{i} \in(0,1)$, we have the expression (4.22), thus we obtain $\log \left|T^{\prime}(x)\right|=\log \beta_{i}+\left(\beta_{i}-\right.$ 1) $\log \left|x-c_{l}\right|$ and $-\log \left|\partial_{y} G(x, y)\right|=\left(1-\alpha_{i}\right) \log \left|x-c_{l}\right|$ with $l=i$ or $l=i+1$.

For the remaining cases, $F \mid\left(c_{i}, c_{i+1}\right) \times \mathbb{I}$ is just a Poincaré map between tubular neighborhoods of regular points for the flow bounded away from equilibria, whose derivatives are bounded: $T^{\prime}$ and $\partial_{y} G$ are bounded functions. In addition, because $\operatorname{det} D F=T^{\prime}(x) \cdot \partial_{y} G(x, y) \neq 0$ we have that $\left|\partial_{y} G(x, y)\right|$ is also bounded away from zero. Since there are finitely many such tubular neighborhoods, we let $K>0$ be an upper bound for these derivatives.

We can now argue exactly as in the proof of Proposition 8 to conclude that the integrals in items 1 and 2 of the statement are finite, since both $\log \left|T^{\prime}(x)\right|$ and $-\log \left|\partial_{y} G(x, y)\right|$ are comparable to the logarithm of the distance to the singular set $\left\{c_{1}, \ldots, c_{n}\right\}$, and this function in integrable, as explained in (4.16). Moreover, since $\left|T^{\prime}\right|>1$ in $\mathbb{I} \backslash\left\{c_{1}, \ldots, c_{n}\right\}$ we also obtain that the integral in item 1 is positive.

Finally, on the one hand, we have seen that $\left|\partial_{y} G(x, y)\right|$ does not depend on $y$ at monotonicity intervals associated to ingoing cross-sections near singularities. On the other hand, on other monotonicity intervals, the function $\left|\partial_{y} G(x, y)\right|$ is bounded above and also away from zero, and it is moreover a $C^{1}$ function with bounded derivatives. This is enough to conclude item 3 of the statement. The proof is complete.

\section{Decay of Correlation for the Poincaré maps.}

Now we are ready to consider the Poincaré maps of singular hyperbolic attractors, and use the results of the previous section to deduce exponential decay of correlations for these maps for each one of the physical measures. To apply Theorem 2 we have to consider a suitable seminorm. We will use var $\square$ as defined at beginning of Section 3 (recall also Theorem 5 item 4). The following Lemma will establish an estimation (see Equation 2.8) which will allow to apply Theorem 2

Lemma 16. If $F$ has the following properties

- $F: Q \rightarrow Q$ is of the form $F(x, y)=(T(x), G(x, y))$ and

- the map uniformly contracts the vertical leaves

- $\operatorname{var}^{\square}(G)<\infty$

- $T: I \rightarrow I$ has $m+1$, increasing branches on the intervals $\left[-\frac{1}{2}=c_{0}, c_{1}\right), \ldots,\left(c_{i}, c_{i+1}\right), \ldots$, $\left(c_{m}, \frac{1}{2}=c_{m+1}\right]$,

If moreover $p \geq 1$, then there are $C, K \in \mathbb{R}$ such that

$$
\left\|\pi\left(f \circ F^{n}\right)\right\|_{1, \frac{1}{p}}+\operatorname{var}^{\square}\left(f \circ F^{n}\right) \leq C K^{n}\left(\|\pi(f)\|_{1, \frac{1}{p}}+\|f\|_{\uparrow l i p}+\operatorname{var}^{\square}(f)\right) .
$$

for each $n \geq 0$.

Before proving the above Lemma, we need the following

Lemma 17. $\|\pi(f)\|_{1,1} \leq 2 \operatorname{var}^{\square}(f)$

Proof. Let us fix $y$ and set $y_{1}=y, \ldots, y_{n}=y$, then

$$
\operatorname{var}^{\square}\left(f, x_{1}, \ldots, x_{n}, y_{1}, \ldots, y_{n}\right)=\sum_{i \leq n}\left|f\left(x_{i}, y\right)-f\left(x_{i+1}, y\right)\right| \leq \operatorname{var}^{\square}(f)
$$

and

$$
\int\left(\sum_{i \leq n}\left|f\left(x_{i}, y\right)-f\left(x_{i+1}, y\right)\right|\right) d y \leq \operatorname{var}^{\square}(f) .
$$

Since

$$
\sum_{i \leq n}\left|\int f\left(x_{i}, y\right) d y-\int f\left(x_{i+1}, y\right) d y\right| \leq \int\left(\sum_{i \leq n}\left|f\left(x_{i}, y\right)-f\left(x_{i+1}, y\right)\right|\right) d y
$$


then

$$
\sum_{i \leq n}\left|\pi(f)\left(x_{i}\right)-\pi(f)\left(x_{i+1}\right)\right| \leq \operatorname{var}^{\square}(f) .
$$

For any $x_{1}, \ldots x_{n}$. By proposition 4 Equation 3.1 we then have the required result.

Proof of Lemma 16. We first remark that

$$
\left\|\pi\left(f \circ F^{n}\right)\right\|_{1, \frac{1}{p}} \leq\left\|\pi\left(f \circ F^{n}\right)\right\|_{1,1} \leq 2 \operatorname{var}^{\square}\left(f \circ F^{n}\right)
$$

so it is sufficient to prove that

$$
3 \operatorname{var}^{\square}\left(f \circ F^{n}\right) \leq C K^{n}\left(\|f\|_{\uparrow l i p}+\operatorname{var}^{\square}(f)\right) .
$$

Let us fix $x_{1} \leq \ldots \leq x_{k}$,

$$
\begin{aligned}
\operatorname{var}^{\square}\left(f \circ F, x_{1}, \ldots, x_{k}, y_{1}, \ldots, y_{k}\right) & =\sum_{i \leq k}\left|f\left(F\left(x_{i}, y_{i}\right)\right)-f\left(F\left(x_{i+1}, y_{i}\right)\right)\right| \\
& =\sum_{i \leq k}\left|f\left(T\left(x_{i}\right), G\left(x_{i}, y_{i}\right)\right)-f\left(T\left(x_{i+1}\right), G\left(x_{i+1}, y_{i}\right)\right)\right| .
\end{aligned}
$$

Suppose that there are no $\left\{c_{i}\right\}$ between $x_{i}$ and $x_{i+1}$. Since $f$ is Lipchitz along the $y$ direction, by the third item in the assumptions

$$
\begin{gathered}
\mid f\left(T\left(x_{i}\right), G\left(x_{i}, y_{i}\right)\right)-f\left(T\left(x_{i+1}\right), G\left(x_{i+1}, y_{i}\right)\right) \leq \\
\left.\leq \mid f\left(T\left(x_{i}\right), G\left(x_{i}, y_{i}\right)\right)\right)-f\left(T\left(x_{i+1}\right), G\left(x_{i}, y_{i}\right)\right)|+|\left|f \|_{\uparrow l i p}\right| G\left(x_{i}, y_{i}\right)-G\left(x_{i+1}, y_{i}\right) \mid .
\end{gathered}
$$

We note that $\sum_{i}\left|G\left(x_{i}, y_{i}\right)-G\left(x_{i+1}, y_{i}\right)\right| \leq \operatorname{var}^{\square}(G)$.

Now suppose that $x_{k_{j}}, \ldots, x_{k_{j+1}-1} \in\left(c_{j}, c_{j+1}\right)$. Since $\left.T\right|_{\left(c_{i}, c_{i+1}\right)}$ is increasing, then

$$
\sum_{k_{j} \leq i \leq k_{j+1}-2}\left|f\left(T\left(x_{i}\right), G\left(x_{i}, y_{i}\right)\right)-f\left(T\left(x_{i+1}\right), G\left(x_{i}, y_{i}\right)\right)\right| \leq \operatorname{var}^{\square}(f)
$$

and

$$
\left|f\left(T\left(x_{k_{j+1}-1}\right), G\left(x_{k_{j+1}-1}, y_{k_{j+1}-1}\right)\right)-f\left(T\left(x_{k_{j+1}}\right), G\left(x_{k_{j+1}-1}, y_{k_{j+1}-1}\right)\right)\right| \leq 2|| f \|_{\infty !} .
$$

Hence, putting all togheter one obtains

$\sum_{i \leq k}\left|f\left(T\left(x_{i}\right), G\left(x_{i}, y_{i}\right)\right)-f\left(T\left(x_{i+1}\right), G\left(x_{i+1}, y_{i}\right)\right)\right| \leq m \cdot \operatorname{var}^{\square}(f)+\|f\|_{\uparrow l i p} v_{a r} \square(G)+2 m\|f\|_{\infty !}$.

This gives

$$
\begin{aligned}
\operatorname{var}^{\square}(f \circ F) & \leq m \cdot \operatorname{var}^{\square}(f)+\operatorname{var}^{\square}(G)\|f\|_{\uparrow l i p}+2 m\|f\|_{\infty !} \\
\operatorname{var}^{\square}\left(f \circ F^{2}\right) & \leq m \cdot \operatorname{var}^{\square}(f \circ F)+\operatorname{var}^{\square}(G)\|f \circ F\|_{\uparrow l i p}+2 m\|f \circ F\|_{\infty !} \\
& =m\left(m \cdot \operatorname{var}^{\square}(f)+\operatorname{var}^{\square}(G)\|f\|_{\uparrow l i p}+2 m\|f\|_{\infty}\right)+\operatorname{var}^{\square}(G)\|f\|_{\uparrow l i p}+2 m\|f\|_{\infty !} \\
\operatorname{var}^{\square}\left(f \circ F^{n}\right) & \leq m^{n} \operatorname{var}^{\square}(f)+\left(m^{n-1}+\ldots+m\right)\left(\operatorname{var}^{\square}(G)\|f\|_{\uparrow l i p}+2 m\|f\|_{\infty !}\right)
\end{aligned}
$$

and the statement is proved.

By Theorem 3 and equation (3.4), we obtain exponential decay of correlations with suitable norms for a class of maps containing the ones found as Poincaré sections of singular hyperbolic flows.

Theorem 8. Let $F: Q \rightarrow Q$ a Borel function such that $F(x, y)=(T(x), G(x, y))$. Let $\mu$ be an invariant measure for $F$ with marginal $\mu_{x}$ on the $x$-axis (which is absolutely continuous and invariant for $T: \mathbb{I} \circlearrowleft)$. Let us suppose that

- $F$ is a contraction on each vertical leaf: $G$ is $\lambda$-Lipschitz in $y$ with $\lambda<1$ for each $x$;

- $\operatorname{var}^{\square}(G)<\infty$;

- $T: I \rightarrow I$ is piecewise monotonic, with $n+1 C^{1}$, increasing branches on the intervals $\left(-\frac{1}{2}, c_{1}\right), \ldots,\left(c_{i}, c_{i+1}\right), \ldots,\left(c_{n}, \frac{1}{2}\right)$ and $i n f_{x}\left(T^{\prime}(x)\right)>1$;

- $\frac{1}{T^{\prime}}$ has finite universal $p$-bounded variation;

- $\mu_{x}$ is weakly mixing. 
Then, $F$ has exponential decay of correlations with respect to suitable norms: there are $C, \Lambda \in$ $\mathbb{R}^{+}, \Lambda<1$

$$
\left|\int g \circ F^{n} \cdot f d \mu-\int g d \mu \int f d \mu\right| \leq C \Lambda^{n}\|g\|_{\uparrow l i p}\left(\|f\|_{\uparrow l i p}+\|\pi(f)\|_{1, \frac{1}{p}}+\operatorname{var}^{\square}(f)\right) .
$$

Since in next section we need to use decay of correlation with respect to lipshitz observables, we show the following estimation.

Lemma 18. Let $f: Q \rightarrow \mathbb{R}$, then

$$
\|\pi(f)\|_{1, \frac{1}{p}} \leq 2 \operatorname{var}^{\square}(f) \leq 2\|f\|_{l i p} .
$$

Proof. Let $f: Q \rightarrow \mathbb{R}$ be Lipschitz. By Lemma 17 we have only to prove $\operatorname{var}^{\square}(f) \leq\|f\|_{l i p}$, but

$$
\operatorname{var}^{\square}\left(f, x_{1}, \ldots, x_{n}, y_{1}, \ldots, y_{n}\right)=\sum_{1 \leq i \leq n}\left|f\left(x_{i}, y_{i}\right)-f\left(x_{i+1}, y_{i}\right)\right| \leq\|f\|_{l i p} \sum_{i}\left|x_{i}-x_{i+1}\right|=\|f\|_{l i p} .
$$

Altogether we obtain exponential decay of correlations with respect to Lipschitz observables, as stated.

Proposition 10. If $F$ satisfies the assumptions of Theorem 8 then it has exponential decay of correlations with respect to Lipshitz observables: there are $C, \Lambda \in \mathbb{R}^{+}, \Lambda<1$ such that

$$
\left|\int g \circ F^{n} \cdot f d \mu-\int g d \mu \int f d \mu\right| \leq C \Lambda^{n}\|g\|_{L i p}\|f\|_{L i p} .
$$

Remark 16. We have seen that if we consider a suitable iterate of the first return map on a section of a flow having a singular hyperbolic attractor, then by Theorem 5 and Remark 8 all the needed assumptions are satisfied. Hence we conclude that this iterate has exponential decay of correlations over lipschitz observables for each of its physical invariant measures.

\section{EXACT DimEnSIONALiTy}

To establish the logarithm law for a singular hyperbolic system, we need to establish that the local dimension exists at a section of the system (see Proposition 111). We recall and use a result of Steinberger [46] and prove that for a singular hyperbolic system, under certain general assumptions the local dimension is defined at almost every point.

Let us consider a map $F: Q \rightarrow Q, F(x, y)=(T(x), G(x, y))$ where

(1) $T:[0,1] \rightarrow[0,1]$ is piecewise monotonic: there are $c_{i} \in[0,1]$ for $0 \leq i \leq N$ with $0=c_{0}<\cdots<c_{N}=1$ such that $T \mid\left(c_{i}, c_{I+1}\right)$ is continuous and monotone for $0 \leq i<N$. Furthermore, for $0 \leq i<N, T \mid\left(c_{i}, c_{i+1}\right)$ is $C^{1}$ and that $\inf _{x \in \mathcal{P}}\left|T^{\prime}(x)\right|>0$ holds where $\mathcal{P}=[0,1] \backslash \cup_{0 \leq i<N} c_{i}$.

(2) $G: Q \rightarrow(0,1)$ is $C^{1}$ on $\mathcal{P} \times[0,1]$. Furthermore, $\sup |\partial G / \partial x|<\infty, \sup |\partial G / \partial y|<1$ and $|(\partial G / \partial y)(x, y)|>0$ for $(x, y) \in \mathcal{P} \times[0,1]$.

(3) $F\left(\left(c_{i}, c_{i+1}\right) \times[0,1]\right) \cap F\left(\left(c_{j}, c_{j+1}\right) \times[0,1]\right)=\emptyset$ for distinct $i, j$ with $0 \leq i, j<N$.

Now consider the projection $\pi_{x}: Q \rightarrow I$, set $\mathcal{V}=\left\{\left(c_{i}, c_{i+1}\right), 1 \leq i \leq N\right\}$ and $\mathcal{V}_{k}=\bigvee_{i=0}^{k} T^{-i} \mathcal{V}$. For $x \in E$ let $J_{k}(x)$ be the unique element of $\mathcal{V}_{k}$ which contains $x$. We say that $\mathcal{V}$ is a generator if the length of the intervals $J_{k}(x)$ tends to zero for $n \rightarrow \infty$ for any given $x$. In piecewise expanding maps it is easy to see that $\mathcal{V}$ is a generator. Set

$$
\psi(x, y)=\log \left|T^{\prime}(x)\right| \quad \text { and } \quad \varphi(x, y)=-\log |(\partial G / \partial y)(x, y)| .
$$

The result of Steinberger that we shall use is the following

Theorem 9. [46, Theorem 1] Let $F$ be a two-dimensional map as above and $\mu_{F}$ an ergodic, $F$-invariant probability measure on $Q$ with the entropy $h_{\mu}(F)>0$. Suppose $\mathcal{V}$ is a generator, 
$\int \varphi \cdot d \mu_{F}<\infty$ and $0<\int \psi d \mu_{F}<\infty$. If the maps $y \mapsto \varphi(x, y)$ are uniformly equicontinuous for $x \in I \backslash\{0\}$ and $1 /\left|T^{\prime}\right|$ has finite universal $p$-Bounded Variation, then

$$
d_{\mu}(x, y)=h_{\mu}(F)\left(\frac{1}{\int \psi \cdot d \mu}+\frac{1}{\int \varphi \cdot d \mu}\right)
$$

for $\mu$-almost all $(x, y) \in Q$.

Now, in the systems we consider, item (3) above is satisfied because the map is induced by a first return Poincaré map induced by a flow; see Remark 13 and Definition 8 in Section 4.2 . Moreover $\sup |\partial G / \partial x|<\infty$ in item (2) above is established at item (6) of Theorem [5. provided that for all equilibria $\sigma \in \Lambda$ we have the eigenvalue relation $-\lambda_{2}(\sigma)>\lambda_{1}(\sigma)$. Let us also observe that, for the first return map $F: Q \backslash \Gamma \rightarrow Q$, associated to the singular-hyperbolic flow, the entropy is positive $h_{\mu}(F)>0$; see Section 4.4 .2 .

So, all we need to prove that $\left(\Xi, F, d \mu_{F}\right)$ is exact dimensional is to verify that $F(x, y)$ satisfies the hypothesis of Theorem 9 . However, Proposition 9 provides precisely that for the functions $\varphi, \psi$ defined above in (6.1): we have

(1) $\int \varphi d \mu_{F}^{i}<\infty$;

(2) $0<\int \psi d \mu_{F}^{i}<\infty$; and

(3) the maps $y \mapsto \varphi(x, y)$ are uniformly equicontinuous for $x \in \mathbb{I} \backslash\left\{c_{1}, \ldots, c_{n}\right\}$;

where $\mu_{F}^{i}$ is each one of the invariant ergodic SRB measure described in Section 4

This all together completes what is necessary to use Theorem 9, establishing that each $\mu_{F}^{i}$ is exact dimensional.

The exact dimensionality of the measure on the section implies the exact dimensionality of the measure $\mu$ on the flow at almost each point, and the dimension satisfies $d_{\mu}(x)=d_{\mu_{F}}(x)+1$ at almost every point $x$.

\section{LOGARITHM LAW FOR SINGULAR HYPERBOLIC ATTRACTORS}

In this section we prove the logarithm law for singular hyperbolic flows. More precisely we prove

Theorem 10. Let $X_{t}: M \circlearrowleft$ be a $C^{2}$ flow having a singular hyperbolic attractor $\Lambda$ satisfying the nonresonance condition in Theorem $\square$ and $-\lambda_{2}(\sigma)>\lambda_{1}(\sigma)$ at each fixed point $\sigma$ of the flow in $\Lambda$. Let us consider its physical invariant measure $\mu$. Let us consider $x_{0}$ and the local dimension at $x_{0}$

$$
d_{\mu}\left(x_{0}\right)=\lim _{r \rightarrow 0} \frac{\log \mu\left(B_{r}\left(x_{0}\right)\right)}{\log r},
$$

(which was above proved to exist almost everywhere), then for $\mu$ almost every $x$

$$
\lim _{r \rightarrow 0} \frac{\log \tau\left(x, B_{r}\left(x_{0}\right)\right)}{-\log r}=d_{\mu}\left(x_{0}\right)-1
$$

where $\tau\left(x, B_{r}\left(x_{0}\right)\right)$ is the time needed for the orbit of $x$ to hit the ball $B_{r}\left(x_{0}\right)$ as defined in (1.2)).

The remaining part of the section is devoted to the proof of the Theorem 10. The strategy is to first establish the law for the Poincaré map associated to the flow, and then extend it to the flow itself. The first step is based on a result about discrete time systems which we recall: let $\left(M, F, \mu_{F}\right)$ be an ergodic, measure preserving transformation on a metric space. In this setting the following is proved in [14] ( see also [15] for a generalization to targets different than balls).

Proposition 11. For each $x_{0}$

$$
\limsup \operatorname{sug}_{r \rightarrow 0} \frac{\log \tau_{F}\left(x, B_{r}\left(x_{0}\right)\right)}{-\log r} \geq \bar{d}_{\mu_{F}}\left(x_{0}\right), \lim _{r \rightarrow 0} \frac{\log \tau_{F}\left(x, B_{r}\left(x_{0}\right)\right)}{-\log r} \geq \underline{d}_{\mu_{F}}\left(x_{0}\right)
$$

hold for $\mu_{F}$-almost every $x$.

Moreover, if the system has super-polynomial decay of correlations under Lipschitz observables and $d_{\mu}\left(x_{0}\right)$ exists, then for $\mu$-almost every $x$ it holds

$$
\lim _{r \rightarrow 0} \frac{\log \tau_{F}\left(x, B_{r}\left(x_{0}\right)\right)}{-\log r}=d_{\mu_{F}}\left(x_{0}\right) .
$$


Remark 17. We show how this result can be applied to deduce a logarithm law for the Poincaré map of singular hyperbolic systems as described in Theorem 5, which is a suitable iteration of the first return map and then to the first return map. By Proposition 10 and Remark 16, we know that the Poincaré map we consider has super-polynomial decay of correlations for Lipshitz observables with respect to each physical invariant measure $\mu_{F}^{i}$. Since in the previous section we proved exact dimensionality of those measures, we can apply Proposition 11 to our Poincaré map.

More precisely, to apply this result to the Poincaré map $F$ and establish the logarithm law, suppose $x_{0}$ is in the basin of $\mu_{F}^{i}$ and consider the system $\left(Q, \mu_{F}^{i}, F\right)$. Now note that since we are dealing with a ratio of logarithms, and (7.3) always hold, if we establish the logarithm law (7.4) for some iterate $F=F_{0}^{n}$, then it will hold also for $F_{0}$; indeed, applying Proposition 11, we know that there is a set $A$ with $\mu_{i}(A)=1$ such that $\lim \sup _{r \rightarrow 0} \frac{\log \tau_{F}\left(x, B_{r}\left(x_{0}\right)\right)}{-\log r} \leq d_{\mu_{F}^{i}}\left(x_{0}\right)$ then we also have $\limsup _{r \rightarrow 0} \frac{\log \tau_{F_{0}}\left(x, B_{r}\left(x_{0}\right)\right)}{-\log r} \leq d_{\mu_{F}^{i}}\left(x_{0}\right)$ for each $x \in A$. But this is also true for each $x \in F_{0}^{-i}(A)$ for each $i$, which by ergodicity of the first return map $F_{0}$ covers a full measure set for the invariant measure $\mu_{F_{0}}$ of the first return map $F_{0}$. Finally we remark that, since each $\mu_{F}^{i}$ is exact dimensional, and each $\mu_{F}^{i}$ give rise to the ergodic physical measure $\mu$ of the flow by suspension, then the a.e. local dimension of each $\mu_{F}^{i}$ will be the same for each $i$.

7.1. Logarithm law for the flow. In Remark 17 we showed that, on the section, the logarithm law holds for the first return map. Let us extend this to the flow. We consider a general measure preserving flow and note that, just like in discrete time systems, one inequality between hitting time behavior and dimension of the measure is valid in general (see [17, Remark 2.4]).

Proposition 12. Let us consider a $C^{1}$ flow preserving the measure $\mu$, then for each $x_{0}$ where $d_{\mu}\left(x_{0}\right)$ is defined

$$
\limsup _{r \rightarrow 0} \frac{\log \tau\left(x, B_{r}\left(x_{0}\right)\right)}{-\log r} \geq \liminf _{r \rightarrow 0} \frac{\log \tau\left(x, B_{r}\left(x_{0}\right)\right)}{-\log r} \geq d_{\mu}\left(x_{0}\right)-1
$$

hold for amost each $x$.

The other inequality can be established by the behavior of the system on a section, as considered before. Let $\Sigma$ be a section of a measure preserving flow $\left(M, \Phi^{t}\right)$. We will show that, if the flow is ergodic and the return time is integrable, then the hitting time scaling behavior of the flow can be estimated by the one of the system induced on the section.

Given any $x \in X$, let us denote by $t(x)$ the smallest strictly positive time such that $\Phi^{t(x)}(x) \in \Sigma$. We also consider $t^{\prime}(x)$, the smallest non negative time such that $\Phi^{t^{\prime}(x)}(x) \in \Sigma$. We remark that these two times differ on the section $\Sigma$, where $t^{\prime}=0$, while $t$ is the return time to the section. We define $\pi: X \rightarrow \Sigma$ as $\pi(x)=\Phi^{t^{\prime}(x)}(x)$, the projection on $\Sigma$. As before denote by $\mu_{F_{0}}$ the invariant measure for the first return Poincare map $F_{0}$ which is induced by the invariant measure $\mu$ of the flow.

Proposition 13 (see [17]). Let us suppose that the flow $\Phi^{t}$ is ergodic and has a transverse section $\Sigma$ with an induced first return map $F_{0}$ and an ergodic invariant measure $\mu_{F_{0}}$ such that

$$
\int_{\Sigma} t(x) d \mu_{F_{0}}<\infty .
$$

Let $r \geq 0$ and $B_{r}\left(x_{0}\right) \subseteq \Sigma$ be balls centered in $x_{0}$, with $\lim _{r \rightarrow 0} \mu_{F_{0}}\left(B_{r}\right)=0$. Then, there is a full measure set $C \subseteq \Sigma$ not depending on $x_{0}$, such that if $x \in C$

$$
\begin{aligned}
\liminf _{r \rightarrow 0} \frac{\log \tau\left(x, B_{r}\left(x_{0}\right)\right)}{-\log r} & =\liminf _{r \rightarrow 0} \frac{\log \tau_{F_{0}}\left(\pi(x), B_{r}\left(x_{0}\right)\right)}{-\log r}, \\
\limsup _{r \rightarrow 0} \frac{\log \tau\left(x, B_{r}\left(x_{0}\right)\right)}{-\log r} & =\limsup _{r \rightarrow 0} \frac{\log \tau_{F_{0}}\left(\pi(x), B_{r}\left(x_{0}\right)\right)}{-\log r} .
\end{aligned}
$$

(We recall that $\tau\left(x, B_{r}\left(x_{0}\right)\right)$ is the time needed for the flow to take $x$ to $B_{r}\left(x_{0}\right)$ and $\tau_{F_{0}}\left(\pi(x), B_{r}\left(x_{0}\right)\right)$ is the time needed for the induced map $F_{0}$ to take $x_{0}$ to the same set.) 
Once we have the logarithm law for the first return map, by this proposition we can extend it to the flow. Indeed, on the section there will be a set $C$ of points where the logarithm law is satistied for each $x \in C$. Let us consider $A=\left\{y \in M: \Phi^{t}(y) \in C\right.$ for some $\left.t \geq 0\right\}$. Then $\mu(A)=1$ (recall that $\mu$ is the physical measure of the flow) because the flow is ergodic and, by definition of $A$, for each $x \in A$ there is $x^{\prime} \in C$ such that $\tau\left(x, B_{r}\left(x_{0}\right)\right) \leq \tau\left(x^{\prime}, B_{r}\left(x_{0}\right)\right)+$ const., where the constant represent the time which is needed for $x$ to arrive in $x^{\prime}$ by the flow, and does not depend on $r$. Hence the same logarithm law which is satisfied on $C$ is satisfied on $A$, showing that, for $x$ in the full measure subset of $A$

$$
\liminf _{r \rightarrow 0} \frac{\log \tau\left(x, B_{r}\left(x_{0}\right)\right)}{-\log r} \leq \limsup _{r \rightarrow 0} \frac{\log \tau\left(x, B_{r}\left(x_{0}\right)\right)}{-\log r} \leq d_{\mu}\left(x_{0}\right)-1 .
$$

A similar construction can be done if the target point $x_{0}$ is not on the section, and extend the result to $x_{0} \in M$. The other inequality is provided by Proposition 12, All together this proves the logarithm law for the flow, once it was proved on the section. This was done in Remark 17] Hence we have established the logarithm law for the flow stated in Theorem 10

\section{Notation of NORMS USED throughout the PAPER}

To help the reader, in this section we give a list of notations related to the various norms which are used in the paper and some explanations to explain their role in the construction.

\subsection{Custom norms used.}

$\|\cdot\|_{\text {I }}:$ the "regularity" norm on the base (in a skew product the norm for which convergence to equilibrium is established for the base transformation).

$\|\cdot\|_{\uparrow l i p}:$ norm evaluating the Lipschitz constant in the vertical direction.

$\|\cdot\|_{\square}$ : a seminorm on the square having some suitable properties with respect to the previous two above norms (see Theorem 2 ).

$v^{\square}{ }^{\square}$ : a sort of bounded variation seminorm on the square (but looking only on increments on the horizontal direction) which is an example of $\|\cdot\|_{\square}$ (for the definition, see beginning of Section 3 ).

$\|\cdot\|_{\infty}$ !: the sup norm on all points (not neglecting zero Lebesgue measure sets, this norm will be used where we have to consider both Lebesgue and the invariant measure, which are singular with respect to each other).

\subsection{Other norms we use or mention.}

$\|\cdot\|_{\infty}$ : the usual $L^{\infty}$ norm with respect to Lebesgue measure.

$\|\cdot\|_{p}$ : the usual $L^{p}$ norm with respect to Lebesgue measure.

$\|\cdot\|_{1}$ : the usual $L^{1}$ norm with respect to Lebesgue measure.

$\|\cdot\|_{p, r}:$ the generalized bounded variation norm with respect to Lebesgue measure (see Section (3).

$\|\cdot\|_{\text {Lip }}:$ the usual Lipschitz norm.

\section{REFERENCES}

[1] R. Abraham and J. Robbin. Transversal mappings and flows. An appendix by Al Kelley. W. A. Benjamin, Inc., New York-Amsterdam, 1967.

[2] V. S. Afraimovich, V. V. Bykov, and L. P. Shil'nikov. On the appearence and structure of the Lorenz attractor. Dokl. Acad. Sci. USSR, 234:336-339, 1977.

[3] V. S. Afraimovich, N. I. Chernov, and E. A. Sataev. Statistical properties of 2-D generalized hyperbolic attractors. Chaos, 5(1):238-252, 1995.

[4] L. Ambrosio, N. Gigli, and G. Savaré. Gradient flows in metric spaces and in the space of probability measures. Lectures in Mathematics ETH Zürich. Birkhäuser Verlag, Basel, second edition, 2008.

[5] D. V. Anosov. Geodesic flows on closed Riemannian manifolds of negative curvature. Proc. Steklov Math. Inst., 90:1-235, 1967.

[6] V. Araújo and M. J. Pacifico. Three-dimensional flows, volume 53 of Ergebnisse der Mathematik und ihrer Grenzgebiete. 3. Folge. A Series of Modern Surveys in Mathematics [Results in Mathematics and Related Areas. 3rd Series. A Series of Modern Surveys in Mathematics]. Springer, Heidelberg, 2010. With a foreword by Marcelo Viana. 
[7] V. Araújo, E. R. Pujals, M. J. Pacifico, and M. Viana. Singular-hyperbolic attractors are chaotic. Transactions of the A.M.S., 361:2431-2485, 2009.

[8] V. Araujo and P. Varandas. Robust exponential decay of correlations for singular-flows. Communications in Mathematical Physics, 311:215-246, 2012.

[9] J. S. Athreya and G. A. Margulis. Logarithm laws for unipotent flows. I. J. Mod. Dyn., 3(3):359-378, 2009.

[10] C. Bonatti, L. J. Díaz, and M. Viana. Dynamics beyond uniform hyperbolicity, volume 102 of Encyclopaedia of Mathematical Sciences. Springer-Verlag, Berlin, 2005. A global geometric and probabilistic perspective, Mathematical Physics, III.

[11] C. Bonatti, A. Pumariño, and M. Viana. Lorenz attractors with arbitrary expanding dimension. C. R. Acad. Sci. Paris Sér. I Math., 325(8):883-888, 1997.

[12] M. Brin and Y. Pesin. Partially hyperbolic dynamical systems. Izv. Acad. Nauk. SSSR, 1:177-212, 1974.

[13] L. A. Bunimovich. Statistical properties of Lorenz attractors. In Nonlinear dynamics and turbulence, pages 71-92. Pitman, 1983.

[14] S. Galatolo. Dimension and hitting time in rapidly mixing systems. Math. Res. Lett., 14(5):797-805, 2007.

[15] S. Galatolo. Hitting time in regular sets and logarithm law for rapidly mixing dynamical systems. Proc. Amer. Math. Soc., 138(7):2477-2487, 2010.

[16] S. Galatolo and D. H. Kim. The dynamical Borel-Cantelli lemma and the waiting time problems. Indag. Math. (N.S.), 18(3):421-434, 2007.

[17] S. Galatolo and I. Nisoli. Shrinking targets in fast mixing flows and the geodesic flow on negatively curved manifolds. Nonlinearity, 24(11):3099-3113, 2011.

[18] S. Galatolo and M. J. Pacifico. Lorenz like flows: exponential decay of correlations for the poincaré map, logarithm law, quantitative recurrence. Ergodic Theory and Dynamical Systems, 30:703-1737, Jan. 2010.

[19] S. Galatolo and P. Peterlongo. Long hitting time, slow decay of correlations and arithmetical properties. Discrete and Continuous Dynamical Systems, 27(1):185-204, 2010.

[20] A. Gorodnik and N. A. Shah Khinchins theorem for approximation by integral points on quadratic varieties Math. Ann., 350, no. 2, pp. 357-380, 2011

[21] N. Gourmelon. Adapted metrics for dominated splittings. Ergodic Theory Dynam. Systems, 27(6):1839-1849, 2007.

[22] J. Guckenheimer and R. F. Williams. Structural stability of Lorenz attractors. Publ. Math. IHES, 50:59-72, 1979.

[23] P. Hartman. Ordinary differential equations, volume 38 of Classics in Applied Mathematics. Society for Industrial and Applied Mathematics (SIAM), Philadelphia, PA, 2002. Corrected reprint of the second (1982) edition [Birkhäuser, Boston, MA; MR0658490 (83e:34002)], With a foreword by Peter Bates.

[24] R. Hill and S. L. Velani. The ergodic theory of shrinking targets. Invent. Math., 119(1):175-198, 1995.

[25] M. Hirsch, C. Pugh, and M. Shub. Invariant manifolds, volume 583 of Lect. Notes in Math. Springer Verlag, New York, 1977.

[26] M. Holland and I. Melbourne. Central limit theorems and invariance principles for Lorenz attractors. J. Lond. Math. Soc. (2), 76(2):345-364, 2007.

[27] C. T. Ionescu-Tulcea and G. Marinescu. Théorie ergodique pour des classes d'operations non complètement continues. Annals of Math., 52:140-147, 1950.

[28] G. Keller. Generalized bounded variation and applications to piecewise monotonic transformations. $Z$. Wahrsch. Verw. Gebiete, 69(3):461-478, 1985.

[29] D. H. Kim and S. Marmi. The recurrence time for interval exchange maps. Nonlinearity, 21(9):2201-2210, 2008.

[30] D. Y. Kleinbock and G. A. Margulis. Logarithm laws for flows on homogeneous spaces. Invent. Math., 138(3):451-494, 1999.

[31] I. Kontoyiannis. Asymptotic recurrence and waiting times for stationary processes. J. Theoret. Probab., 11(3):795-811, 1998.

[32] E. N. Lorenz. Deterministic nonperiodic flow. J. Atmosph. Sci., 20:130-141, 1963.

[33] R. Metzger and C. Morales. Sectional-hyperbolic systems. Ergodic Theory and Dynamical System, 28:1587$1597,2008$.

[34] C. A. Morales and M. J. Pacifico. A dichotomy for three-dimensional vector fields. Ergodic Theory Dynam. Systems, 23(5):1575-1600, 2003.

[35] C. A. Morales, M. J. Pacifico, and E. R. Pujals. Robust transitive singular sets for 3-flows are partially hyperbolic attractors or repellers. Ann. of Math. (2), 160(2):375-432, 2004.

[36] J. Palis and W. de Melo. Geometric Theory of Dynamical Systems. Springer Verlag, 1982.

[37] J. Palis and F. Takens. Hyperbolicity and sensitive-chaotic dynamics at homoclinic bifurcations. Cambridge University Press, 1993.

[38] Y. Pesin. Dynamical systems with generalized hyperbolic attractors: hyperbolic, ergodic and topological properties. Ergod. Th. ES Dynam. Sys., 12:123-151, 1992.

[39] M. Pollicott. On the rate of mixing of Axiom A flows. Invent. Math., 81(3):413-426, 1985.

[40] C. Pugh and M. Shub. Ergodicity of Anosov actions. Invent. Math., 15:1-23, 1972.

[41] C. Pugh, M. Shub, and A. Wilkinson. Hölder foliations. Duke Math. J., 86:517-546, 1997. 
[42] C. Robinson. Dynamical systems. Studies in Advanced Mathematics. CRC Press, Boca Raton, FL, Second edition, 1999. Stability, symbolic dynamics, and chaos.

[43] D. Ruelle. Flots qui ne mélangent pas exponentiellement. C. R. Acad. Sci. Paris Sér. I Math., 296(4):191-193, 1983.

[44] P. C. Shields. Waiting times: positive and negative results on the Wyner-Ziv problem. J. Theoret. Probab., 6(3):499-519, 1993.

[45] M. Shub. Global stability of dynamical systems. Springer Verlag, 1987.

[46] T. Steinberger. Local dimension of ergodic measures for two-dimensional lorenz transformations. Ergodic Theory and Dynamical Systems, 20(3):911-923, 2000.

[47] D. Sullivan. Disjoint spheres, approximation by imaginary quadratic numbers, and the logarithm law for geodesics. Acta Math., 149(3-4):215-237, 1982.

[48] L. S. Young. Statistical properties of dynamical systems with some hyperbolicity. Annals of Math., 147:585650, 1998.

(V.A.) Instituto de Matemática, Universidade Federal da Bahia, Av. Adhemar de Barros, S/N, Ondina, 40170-110 - SAlvador-BA-BraziL

E-mail address: vitor.d.araujo@ufba.br

(S.G.) Dipartimento di Matematica Applicata, Via Buonarroti 1 Pisa

E-mail address, S. Galatolo: galatolo@dm.unipi.it

URL: http://users.dma.unipi.it/galatolo/

(M.J.) Instituto de Matemática, Universidade Federal do Rio de Janeiro, C. P. 68.530, 21.945-970 Rio DE JANEIRO

E-mail address: pacifico@im.ufrj.br 